Johannes Kamp*, Jörn Villwock and Matthias Kraume

\title{
Drop coalescence in technical liquid/liquid applications: a review on experimental techniques and modeling approaches
}

DOI 10.1515/revce-2015-0071

Received December 2, 2015; accepted May 10, 2016

Abstract: The coalescence phenomenon of drops in liquid/liquid systems is reviewed with particular focus on its technical relevance and application. Due to the complexity of coalescence, a comprehensive survey of the coalescence process and the numerous influencing factors is given. Subsequently, available experimental techniques with different levels of detail are summarized and compared. These techniques can be divided in simple settling tests for qualitative coalescence behavior investigations and gravity settler design, single-drop coalescence studies at flat interfaces as well as between droplets, and detailed film drainage analysis. To model the coalescence rate in liquid/liquid systems on a technical scale, the generic population balance framework is introduced. Additionally, different coalescence modeling approaches are reviewed with ascending level of detail from empirical correlations to comprehensive film drainage models and detailed computational fluid and particle dynamics.

Keywords: coalescence; coalescence model; emulsion; liquid/liquid dispersion; population balance equation.

\section{Abbreviations}

$\begin{array}{ll}\text { 1D } & \text { one-dimensional } \\ \text { 2D } & \text { two-dimensional } \\ \text { 3D } & \text { three-dimensional } \\ \text { AC } & \text { alternating current } \\ \text { AFM } & \text { atomic force microscopy } \\ \text { CFD } & \text { computational fluid dynamics }\end{array}$

*Corresponding author: Johannes Kamp, Chair of Chemical and Process Engineering, Technische Universität Berlin, Straße des 17. Juni 135, FH 6-1, 10623 Berlin, Germany, e-mail: Johannes.Kamp@tu-berlin.de. http://orcid.org/0000-0003-1778-7607

Jörn Villwock and Matthias Kraume: Chair of Chemical and Process Engineering, Technische Universität Berlin, Straße des 17. Juni 135, FH 6-1, 10623 Berlin, Germany. http://orcid.org/0000-0002-2958-0817 (J. Villwock)

$\begin{array}{ll}\mathrm{c} \rightarrow \mathrm{d} & \text { mass transfer from continuous to disperse phase } \\ \mathrm{d} \rightarrow \mathrm{c} & \text { mass transfer from disperse to continuous phase } \\ \text { DC } & \text { direct current } \\ \text { DIM } & \text { diffusive interface method } \\ \text { DLVO } & \text { Derjaguin-Landau-Verwey-Overbeek } \\ \text { fps } & \text { frames per second } \\ \text { HLB } & \text { hydrophilic-lipophilic balance concept } \\ \text { LBM } & \text { lattice Boltzmann method } \\ \text { LED } & \text { light-emitting diode } \\ \text { MD } & \text { molecular dynamics } \\ \text { O/W } & \text { oil-in-water emulsion/dispersion } \\ \text { PBE } & \text { population balance equation } \\ \text { PIV } & \text { particle image velocimetry } \\ \text { SFA } & \text { surface force apparatus } \\ \text { TFB } & \text { thin-film balance } \\ \text { VOF } & \text { volume of fluid } \\ \text { W/O } & \text { water-in-oil emulsion/dispersion }\end{array}$

\section{Introduction}

The coalescence or confluence of drops or bubbles with one another or a continuous phase is a phenomenon that does affect technical applications but also influences everyday life. For example, without coalescence, rain drops would not flow together to runlets and puddles, but millions of drops would accumulate on the ground. As most of the chemicals used in industrial applications are liquids and gases, the technical relevance of coalescence for chemical and process engineering becomes apparent. In liquid/ liquid systems, (at least) two phases with a miscibility gap are dispersed in one another by an energy input to overcome the density difference and to, for example, increase the interfacial area and to enhance the mass and heat transfer processes. Those dispersed systems are an integral part of many technical applications (e.g. extraction, mixing, polymerization, emulsion formation, and multiphase reactors). In other cases or production steps, stabilized dispersions (or emulsions) have to be separated again. The rate-determining phenomenon in separation is solely the coalescence rate of the dispersed droplets with each other and with their continuous phase. The most relevant 
example is the separation of water-in-crude oil dispersions that are recovered from oil wells. The water has to be separated before the crude oil can be further processed in petroleum and chemical industries. However, coalescence is not always desired: for example, in cosmetic products, such as lotions and creams in which water and oil are finely dispersed, as well as in food and nutrition products, such as milk and margarine. In these cases, the stabilized dispersion is a demanded product quality; therefore, coalescence is significantly hindered by surface-active additives to prevent the separation into two continuous phases. Hence, the coalescence rate and probability may and often should vary significantly from application to application; consequently, its influencing parameters have to be understood.

The drop size distribution in liquid/liquid dispersions determines the interfacial area and the sedimentation velocity during separation. Thus, it is an important process variable and determines a significant part of the overall process efficiency and product quality. In technical applications, drops typically do not appear monodisperse, but a droplet swarm with a certain spectrum of different drop sizes is formed by drop breakage and coalescence events. The polydisperse droplet swarm is characterized by the drop size distribution. To predict drop size distributions, the opposing phenomena of drop coalescence and breakage have to be understood and described properly. Although comprehensive scientific research identified a multitude of theoretical models describing both processes, the prediction of the drop size distribution is only possible with restrictions up to now. Consequently, excessive and expensive experimental investigations are still necessary at different scales of process development. For example, the design of extraction columns still requires pilot plants using a significant amount of (possibly toxic, hazardous, and/or valuable) original process liquids.

\subsection{Scope}

This work is focused on the droplet coalescence phenomenon in liquid/liquid systems. Concerning drop breakage, the reader is referred to other works (Lasheras et al. 2002, Liao and Lucas 2009, Ghotli et al. 2013, Solsvik et al. 2013, Nachtigall et al. 2015). The review is written from the perspective of an engineer summarizing the complex physical interactions and how these findings are integrated in models describing technical apparatuses. It is divided in three parts. First, an overview of the fundamental steps of droplet coalescence and its influencing factors is given. In the second part, available experimental techniques with different levels of detail are summarized and compared. Additionally, major outcomes are presented. The last part reviews and discusses existing modeling approaches from empirical correlations to comprehensive film drainage models and detailed computational fluid and particle dynamics.

At the end of each part, short intermediate conclusions are given in which the authors summarize the main outcomes and evaluate the discussed aspects subjectively.

Coalescence in gas/liquid systems is not considered in detail in this review. Coalescence in gas/liquid systems is similar to coalescence in liquid/liquid systems but not equivalent: the dynamic viscosity of the gas phase is orders of magnitude lower (and thus negligible) and surfaceactive substances cannot be dissolved in gases. Therefore, the parameters of the liquid phase mostly influence the coalescence process and the gas/liquid coalescence can be regarded as a limiting case of liquid/liquid coalescence. However, the coalescence of gas bubbles in liquids is more similar to liquid/liquid coalescence than the coalescence of drops in gas continuous phase because the coalescence process is mainly determined by the viscous continuous phase. In case of drop coalescence in gas continuous phase, the coalescence event can be described successfully depending on the drop size ratio, Weber number, and collision angle (Bradley and Stow 1978, Ashgriz and Poo 1990, Orme 1997, Qian and Law 1997, Estrade et al. 1999, Chen and Chen 2006, Pan et al. 2009).

\subsection{Early history}

Coalescence itself is a highly complex phenomenon that has been investigated systematically since the first half of the 20th century but still is in the focus of recent research. Looking at the early beginnings of coalescence investigation, the first scientific description was made by Reynolds (1881), who splashed water on ponds and observed droplets resting and floating on the water surface until confluence appeared. The drop floating effect was later reported again by Katalinic (1926). Reynolds (1881) explained the floating of drops by the purity of the surface resulting in high interfacial tension. Prior to that, Reynolds (1875) already used colored drops to visualize the vortex ring evolving within the continuous phase after coalescence. The vortex rings were also described by Thomson and Newall (1885). A phenomenological description of the film thinning (or drainage) above a bubble approaching the water surface, which is the most important step in the coalescence process, was given by Hardy (1925). The first systematic investigations of drop and bubble rest times at a flat interface until coalescence occurred were performed by Rehbinder and Wenström (1930) and Rehbinder et al. (1930), who also analyzed the adsorption and stabilizing effect of surface-active 
components. Mahajan (1933) investigated the thin film of continuous oil phase between water droplets. Wark and Cox (1935) reported interdrop coalescence in several stages (also known as partial coalescence). A detailed insight in the film thinning was reported by Derjaguin and Kussakov (1939), who performed the first measurements of the film thickness during thinning at a plane interface. Derjaguin and Kussakov (1939) postulated that the thermodynamic properties within the thin film differ from those of the bulk phase and termed the corresponding difference disjoining pressure (Sheludko 1967). The first mechanistic approach of coalescence was described by Lawrence and Mills (1954), who applied the coagulation theory of Smoluchowski (1918) to oil-in-water emulsions (O/W). In the following decades, a huge number of publications can be found investigating coalescence phenomena in liquid/ liquid systems, which are discussed in this work.

\section{Fundamentals of drop coalescence}

Due to the complexity of coalescence, the fundamentals of the coalescence process and its numerous influencing factors are introduced in detail before experimental techniques and modeling approaches are discussed in the subsequent sections. First, the collision of two drops due to their relative velocity in different flow regimes is summarized. Subsequently, a comprehensive survey of the sequential steps during the collision of two drops is given, which can result in coalescence, agglomeration, or repulsion. Additionally, various parameters that influence the coalescence probability of two drops in contact are discussed.

\subsection{Droplet collision}

Before a coalescence of two droplets can occur, the drops have to collide and come into contact with each other. The collision between two droplets is induced by different drop velocities $v$ compared to the surrounding continuous flow field, resulting in a relative velocity $v_{\text {rel }}$ between the droplets. The relative velocity is determined mainly by the main flow pattern of the dispersion and the settling (or rising) velocities of the individual drops. In technical applications, a broad variety of flow regimes can be found from stagnant continuous phase to fully turbulent flows (see Table 1); for example, in a gravity separator, a sedimentation zone with settling or rising droplets and a dense-packed zone of resting drops in the dispersion band can be found. The rise velocity of a settling drop can be calculated by a force balance around the droplet using the friction factor $C_{D S}$ for single droplets (see Wegener et al. 2014 for details) or the friction factor $C_{D, \varphi}$ corrected by the disperse phase fraction $\varphi$ for droplet swarms (see Barnea and Mizrahi 1973, 1975a, Henschke et al. 2002). The relative velocity between two droplets depends only on the drop sizes if velocity gradients of the continuous phase flow are neglected, otherwise complex interactions have to be considered as derived by Berman and Tamir

Table 1: Relative velocities between two drops in technical applications in different flow regimes.

\begin{tabular}{|c|c|c|}
\hline Flow regime & Velocity & Reference \\
\hline Dense-packed zone & $v_{\mathrm{rel}} \approx 0$ & Barnea and Mizrahi 1975b,c \\
\hline Gravity settling & $v=\sqrt{\frac{4}{3} \frac{\left|\rho_{d}-\rho_{c}\right|}{\rho_{c}} g d \frac{1}{C_{D}}}$ with & \\
\hline Single droplet & $C_{D, s}=f\left(\operatorname{Re}, \mu_{c}, \mu_{d}\right), \operatorname{Re}=\frac{v d \rho_{c}}{\mu_{c}}$ & Wegener et al. 2014 \\
\hline Dispersion & $C_{D, \varphi}=f\left(\operatorname{Re}_{\varphi}, \varphi, \mu_{c}, \mu_{d}\right), R e_{\varphi}=\frac{v d \rho_{c}}{\mu_{\varphi}}, \mu_{\varphi}=f\left(\varphi, \mu_{c}, \mu_{d}\right)$ & $\begin{array}{l}\text { Barnea and Mizrahi 1973, 1975a, } \\
\text { Henschke et al. } 2002\end{array}$ \\
\hline Viscous shear & $v_{\mathrm{rel}}=\dot{\gamma} d$ & $\begin{array}{l}\text { Chesters 1991, Smoluchowski 1918, } \\
\text { Batchelor and Green } 1972\end{array}$ \\
\hline $\begin{array}{l}\text { Capture in larger } \\
\text { turbulent eddy }\end{array}$ & $v_{\mathrm{rel}}=\left(\frac{2 \epsilon \rho_{c}}{15 \mu_{c}}\right)^{1 / 2} d$ & $\begin{array}{l}\text { Liao and Lucas 2010, Chesters } 1991, \\
\text { Saffman and Turner } 1956\end{array}$ \\
\hline $\begin{array}{l}\text { Locally isotropic and } \\
\text { homogeneous turbulence }\end{array}$ & $v_{\mathrm{rel}}=\sqrt{2}(\epsilon d)^{1 / 3}$ & $\begin{array}{l}\text { Kolmogorov 1941, Hinze 1955, Coulaloglou } \\
\text { and Tavlarides 1977, Batchelor 1951, 1952, } \\
\text { Kuboi et al. 1972a,b }\end{array}$ \\
\hline
\end{tabular}


(2003). The relative velocity in the dense-packed zone is nearly zero, although the coalescence events induce fluctuations and movement in the dispersion band. The absolute velocity of the droplets is determined by the superficial velocity (or surface load) of the separator (Barnea and Mizrahi 1975b,c). In viscous shear flow (e.g. pipe flow), the relative velocity is determined by the shear rate $\dot{\gamma}$ and a characteristic distance of the order of magnitude of one droplet diameter $d$. Drops following the confined streamlines of the external shear flow can only approach each other if the diameter of the smaller droplet is at least 15\% of the bigger one (Smoluchowski 1918, Batchelor and Green 1972, Chesters 1991). In turbulent flow regimes (e.g. stirred vessel, extraction column, pipe flow), drops are entrained in eddies larger than the drops itself; only eddies in the scale of the drops can cause a relative velocity between them. Thus, the relative velocity can be derived from the energy distribution theory of Kolmogorov (1941) in isotropic homogeneous turbulence (Batchelor 1951, 1952, Hinze 1955, Kuboi et al. 1972a,b, Coulaloglou and Tavlarides 1977). If the drop sizes are much smaller than the energy dissipating eddies in the turbulent flow, the relative velocity can be calculated from the shear strain (or turbulent shear) rate within the smallest eddy (Saffman and Turner 1956, Chesters 1991, Liao and Lucas 2010).

A discussion of the typical forces and contact times of colliding particles in the mentioned flow regimes can be found in the work of Chesters (1991). From these, the collision frequency $\xi$ can be derived, which describes how often drops collide within the existent flow regime. Several models describing the collision frequency can be found in the works of Chesters (1991) and Liao and Lucas (2010).

After a collision of two droplets, a coalescence of these may occur. This is determined by a coalescence probability (or efficiency) $\lambda$, which depends on complex droplet interactions and influencing parameters, as discussed in the following. Together with the collision frequency $\xi$, the coalescence probability $\lambda$ yields the coalescence rate $F$ in physical modeling approaches:

$$
F=\xi \cdot \lambda .
$$

The coalescence rate describes how often drops coalesce within the existent flow.

\subsection{Coalescence process}

The collision between two droplets can subsequently result in coalescence, agglomeration, or repulsion of the drops. This process can be divided in several stages (Jeffreys and Davies 1971, Vijayan and Ponter 1974):
I. Approach
II. "Contact” and deformation drop interfaces
IVa. Coalescence
1. Film rupture
2. Forming of coalescence bridge
3. Confluence

III. Drainage of continuous film trapped between

IVb. Agglomeration

IVc. Repulsion

These four main stages, which are described more detailed in the following, can be observed during every collision, but the occurrence and their time span may differ significantly with chemical and process conditions (see Section 2.3). The numerous influencing factors determine if the collision between two drops results in coalescence, agglomeration, or repulsion.

The collision of a drop with a planar interface is the limiting case if one drop radius becomes infinity and thus, to some extent, comparable to the collision of two droplets with finite radius. Hence, early reports (Gillespie and Rideal 1956, Charles and Mason 1960a,b, Allan et al. 1961, MacKay and Mason 1963a,b, Jeffreys and Hawksley 1965a, Hartland 1967a,b,c, 1969) mostly investigated droplet/ flat interface collisions due to simplicity of experimental set-up and observability (Scheele and Leng 1971). To consider the collision of two unequally sized drops with diameter $d_{1}$ and $d_{2}$ (see Figure 1), the equivalent diameter

$$
d_{e q}=\frac{2 d_{1} d_{2}}{d_{1}+d_{2}}
$$

is most widely used. It can be derived from the YoungLaplace equation (Princen 1969, Butt et al. 2003) and indicates that the coalescence of a droplet of diameter $d^{*}$ with a flat interface should be equivalent to the coalescence of two equally sized drops of diameter $2 d^{*}$ (Chesters 1991). A further discussion and detailed derivation of droplet interactions with unequal diameters can be found in the works of Yiantsios and Davis (1991), Abid and Chesters (1994), and Kamp et al. (2001).

The possibly occurring interactions between deformable drops and bubbles on collision were discussed in detail by Danov et al. (1993) and Kralchevsky et al. (2008). The mentioned stages of droplet collision are shown schematically in Figure 2. A high-speed recording of coalescing drops is shown in Figure 3. The discussed time spans may 

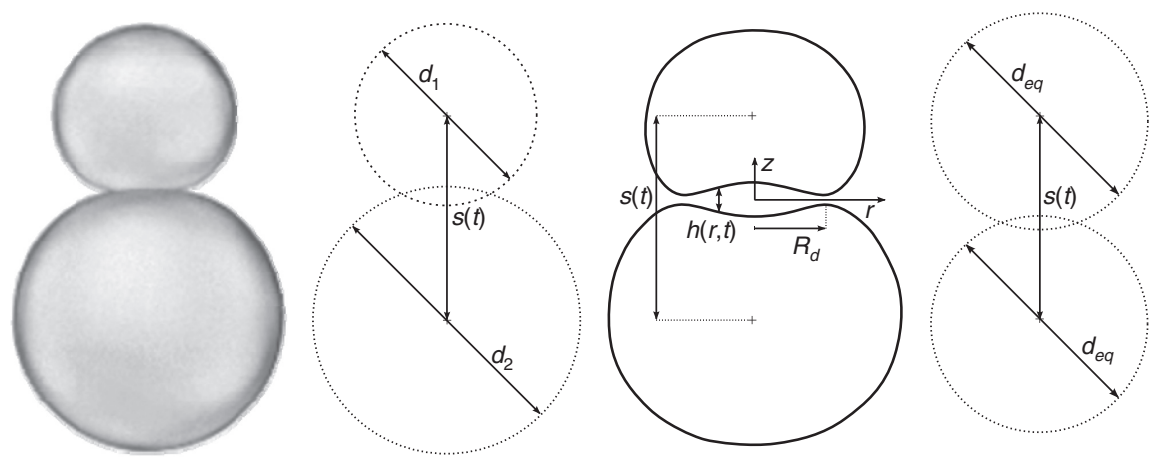

Figure 1: Collision and drainage film formation $h(r, t)$ between two unequally sized droplets (diameter $d_{1} \& d_{2}$ ) and the collision of two drops with equivalent diameter $d_{e q}$ according to Equation (2), all centers of mass separated by distance $s(t)$.

Adapted from Lee and Hodgson (1968) and Yiantsios and Davis (1991).

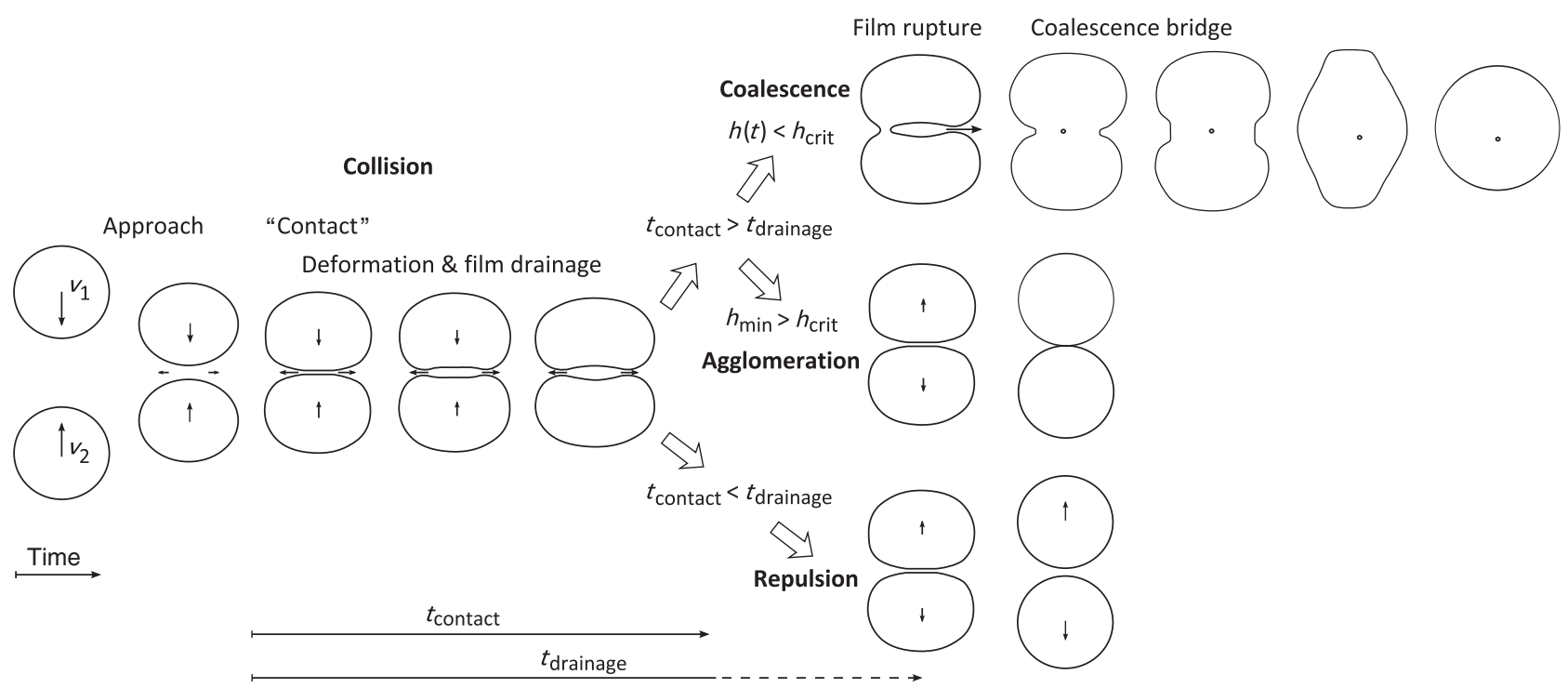

Figure 2: Schematic sequence of the collision of two droplets and subsequent coalescence, agglomeration, or repulsion. Adapted from Gäbler (2007).

occasionally have different definitions in the referenced literature (especially the coalescence time); thus, the time spans are defined in the following description for this work.

I. Approach: The droplets approach each other with a relative velocity (here, $v_{\mathrm{rel}}=v_{1}+v_{2}$ ); however, the collision is not necessarily head-on as depicted here. By comparison of head-on and eccentric collisions, Borrell et al. (2004) found the film drainage process of frontal collisions being concordant with the drainage of eccentric collisions. Accordingly, we focus on the head-on collision in this scheme. The two droplets displace the continuous phase in front of them, which induces a frontal wave. At a certain separation distance $s$, the two frontal waves interact with each other, which would result in an increased displacement of continuous phase that is limited by viscous forces. To counterbalance this, a deceleration and deformation of the droplets appears. Due to the deformation, a thin film of continuous phase is enclosed between the two droplets (see Figure 1; Hardy 1925, Mahajan 1933). Guido and Simeone (1998) and Tretheway et al. (1999) investigated the trajectories of colliding spheres and deformable droplets. From these investigations, it can be determined that the interaction of two equally sized drops begins at a center of mass distance of around three times the radius; thus, for unequally sized drops, the interaction distance yields:

$$
s_{\text {interaction }}=\frac{3}{4}\left(d_{1}+d_{2}\right)
$$




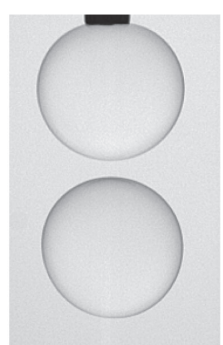

$$
-69.83 \mathrm{~ms}
$$

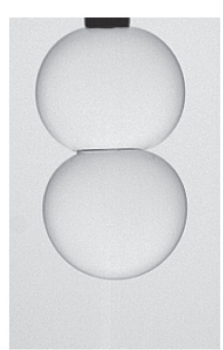

$5.73 \mathrm{~ms}$

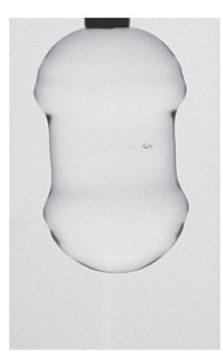

$7.73 \mathrm{~ms}$

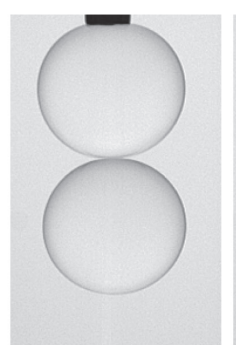

$0 \mathrm{~ms}$

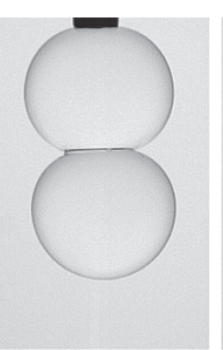

$5.76 \mathrm{~ms}$

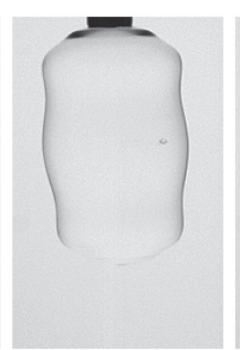

$9.32 \mathrm{~ms}$

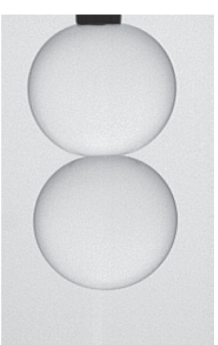

$2.97 \mathrm{~ms}$

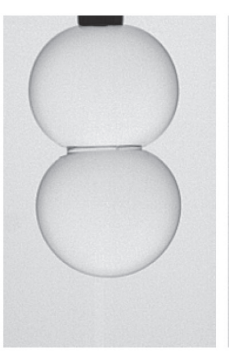

$5.82 \mathrm{~ms}$

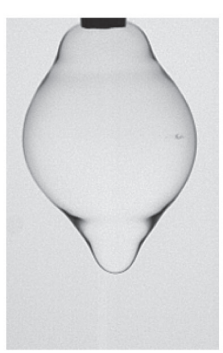

$12.49 \mathrm{~ms}$

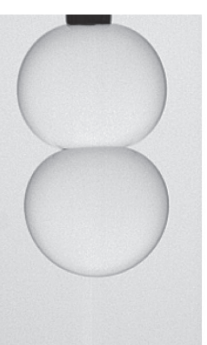

$5.63 \mathrm{~ms}$

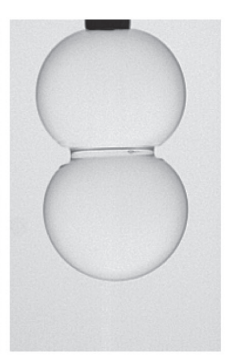

$5.98 \mathrm{~ms}$

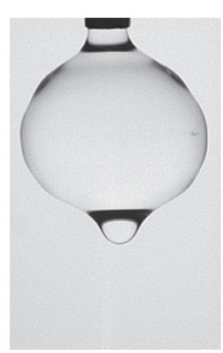

$14.55 \mathrm{~ms}$

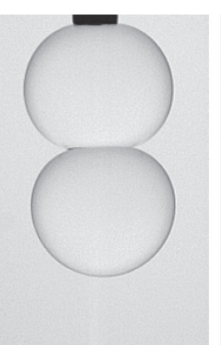

$5.65 \mathrm{~ms}$

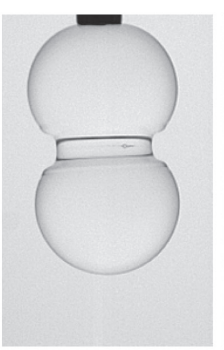

$6.30 \mathrm{~ms}$

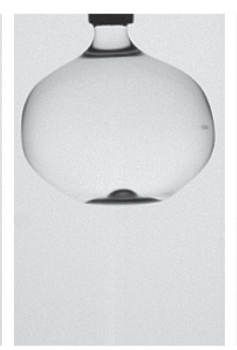

$16.14 \mathrm{~ms}$

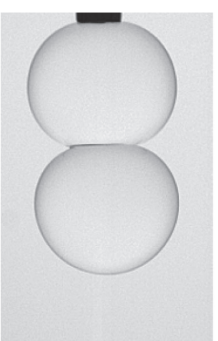

$5.70 \mathrm{~ms}$

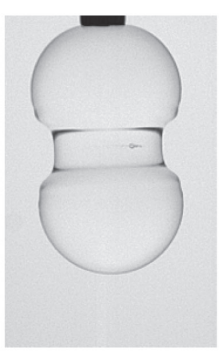

$6.78 \mathrm{~ms}$

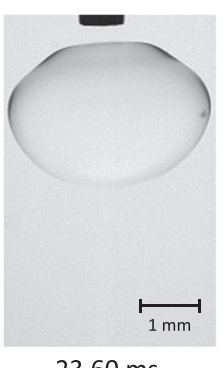

$23.60 \mathrm{~ms}$

Figure 3: Coalescence of two toluene drops $\left(d_{\text {top }}=2.6 \mathrm{~mm}, d_{\text {bottom }}=2.5 \mathrm{~mm}\right)$ in water recorded with Vision Research Phantom v711 at a frame rate of $63,021 \mathrm{fps}$.

After drop deformation and film drainage, film rupture occurs $5.63 \mathrm{~ms}$ after visual contact on the left-hand side of the film, which lasts for about $0.1 \mathrm{~ms}$. Thereby, a small droplet of continuous phase from the draining film is enclosed in the coalesced drop. The coalescence bridge is built and the drops confluence.

II. "Contact" and deformation: The "contact" between the drops (and start of film drainage) was defined by Baldessari and Leal (2006) as the moment at which the undeformed droplets would touch each other; thus, the separation distance between the two centers of mass is equal to:

$$
S_{\text {contact }}=\frac{1}{2}\left(d_{1}+d_{2}\right)
$$

In optical observations, the continuous phase between the droplets becomes invisible in this moment and the drop surfaces seem to touch each other (Tretheway et al. 1999, Villwock et al. 2014a). Driven by inertia force, the droplets tend to approach each other further and become more deformed.

III. Film drainage: To minimize the film thickness $h$ between the drops, the enclosed film with radius $R_{d}$ has to drain (see Figure 1). This flow is induced by the pressure drop from the middle of the film to the periphery of the droplets (Chappelear 1961). A capillary pressure is caused within the enclosed film by a curvature of the drop interface. Together with the van der Waals component of the disjoining pressure the capillary pressure generates this pressure drop (Sheludko 1967). This leads to an increase of the film thickness as one moves from the edge of the film to the center. The flow speed at the circular rim of the enclosed film is maximal and causes a decrease in static pressure, which converges the drop interfaces at this circular boundary additionally (Jones and Wilson 1978, Klaseboer et al. 2000). Due to these complex interactions, the enclosed film has not a discoid but typically a lenticular shape, which is called a dimple (Charles and Mason 1960b, Allan et al. 1961, MacKay and Mason 1963b, Princen 1963, Jeffreys and Hawksley 1965b). However, the film drainage does not need to occur symmetrically: several investigations found that drainage is initially 
axisymmetric and might spontaneously develop asymmetric pattern (Hartland 1967b, Sheludko 1967, Burrill and Woods 1973a,b, Velev et al. 1995, Zdravkov et al. 2006, Chan et al. 2011). It is assumed that this asymmetry of drainage is associated with spatial variations of surfactants and locally mobile interfaces might occur (Burrill and Woods 1973a). However, the mechanisms that trigger asymmetric film drainage are still the object of research. If the kinetic energy of the two droplets becomes zero and the deformation reaches a maximum, the interfacial tension contracts the drops back to spherical shape, which moves the centers of mass apart from each other again. However, film drainage can still occur for a certain period of time. The time span from the "contact" of the droplets until the end of the film drainage is called film drainage time $t_{\text {drainage }}$ after which a minimal film thickness $h_{\text {min }}$ at the circular rim of the film is reached between the two droplet surfaces. Obviously, coalescence or agglomeration can only occur if the total contact time $t_{\text {contact }}$ of the two drops exceeds the drainage time $t_{\text {drainage }}$. Otherwise, the collision ends in a repulsion of the droplets. The drainage time depends on many influencing factors (see Section 2.3) and thus has a broad variation from milliseconds (Scheele and Leng 1971, Sagert and Quinn 1978) over seconds (Vijayan and Ponter 1975) to infinity for stable emulsions (Carroll 1976, Davis and Smith 1976). This is discussed in detail in the subsequent sections.

IVa. Coalescence: To result in coalescence, the distance $h$ between the two drop surfaces has to fall below a critical distance along the rim $h_{\text {crit }}(r, t)=h\left(R_{d}, t_{\text {drainage }}\right)$ at some point during the film drainage (Hardy 1925, Mahajan 1933, Charles and Mason 1960b). Below this critical distance $h_{\text {crit }}$, the surfaces spontaneously confluence at one (or several) point(s) and a conjunction is built (de Vries 1958a, Charles and Mason 1960b, Aarts et al. 2005, Zdravkov et al. 2006, Aarts and Lekkerkerker 2008). Gillespie and Rideal (1956) and Charles and Mason (1960a) proposed surface waves in the thin film to be responsible for the spontaneous film rupture (Vrij 1966, Vrij et al. 1970), which could be measured directly about 50 years later using laser scanning confocal microscopy by Aarts et al. (2004). Although van der Waals attraction is assumed to be the main cause for the film break-up (Chen et al. 2004), Aarts et al. (Aarts et al. 2005, Aarts and Lekkerkerker 2008) found film rupture induced by thermal capillary waves in a system in which van der Waals forces are small. In contrast, Manica et al. (2008b) doubted that thermal or capillary fluctuations play a role in the coalescence process. Zdravkov et al. (2006) explained the instability of the film by the overlap of diffusive interfacial layers (diffusing molecules of disperse phase in the continuous phase), which is induced by Marangoni convection and surface waves. Although the distinct processes of film rupture are still in the focus of current research, it is quite evident that local interactions at small scale dominate the film rupture. Therefore, the moment and point at which film rupture occurs cannot be described explicitly, but stochastic approaches must be used (Radoev et al. 1983). The values for the critical film rupture thickness $h_{\text {crit }}$ which can be found in the literature, differ from tens (de Vries 1958a, Scheludko et al. 1965, Vrij 1966, Burrill and Woods 1973a, Radoev et al. 1983) to hundreds of nanometers (Gillespie and Rideal 1956, Zdravkov et al. 2003), whereas theoretical analyses predict a range of only few nanometers (Vrij 1966, Chesters 1991). Reviews on thin films, including film rupture and flow inside the film, can be found in the works of de Vries (1958a,b,c,d), Lee and Hodgson (1968), and Clunie et al. (1971). However, occasionally coalescence can be observed in the moment when the drops begin to separate again. In these cases, the pressure and flow conditions within the film change the interface curvature and cause an approach and rupture of the film at its center (Bremond et al. 2008).

From the conjunction of the disperse phases [little hole(s) in the film], the interfacial tension rapidly enlarges the link along the circular border of the film and expels the remaining film volume (film rupture time $t_{\text {rupture }}$; Charles and Mason 1960b, MacKay and Mason 1963b, Aarts et al. 2005, Aryafar and Kavehpour 2006, Zdravkov et al. 2006, Aarts and Lekkerkerker 2008). This is shown in a high-speed sequence in Figure 4. The film rupture time $t_{\text {rupture }}$ varies from hundreds of microseconds to milliseconds (Aryafar and Kavehpour 2006) depending on the viscosities of both phases. If the conjunction of the surfaces along the circular border is faster than the expulsion of the film volume, the remaining continuous phase will be enclosed as droplet(s) inside the coalesced disperse phase (Charles and Mason 1960b), which is also depicted in Figures 2 and 3. When the drops are connected along the region where the film of continuous phase has been located before, a characteristic coalescence bridge is formed along which the drops confluence (de Vries 1958a, Scheludko et al. 1965, Vrij 1966, Radoev et al. 1983, Chen et al. 2004, Thoroddsen et al. 2005). The interplay of inertia, viscous, and interfacial forces induces oscillations until the surface waves are dampened and the newly formed drop has a spherical shape. Coalescence time is mostly defined as the sum of drainage time and film rupture time $t_{\text {coalescence }}=t_{\text {drainage }}+t_{\text {rupture }}$ and thus the time interval from "contact" until start of confluence. In general, the drainage time is much larger than the film rupture time. 


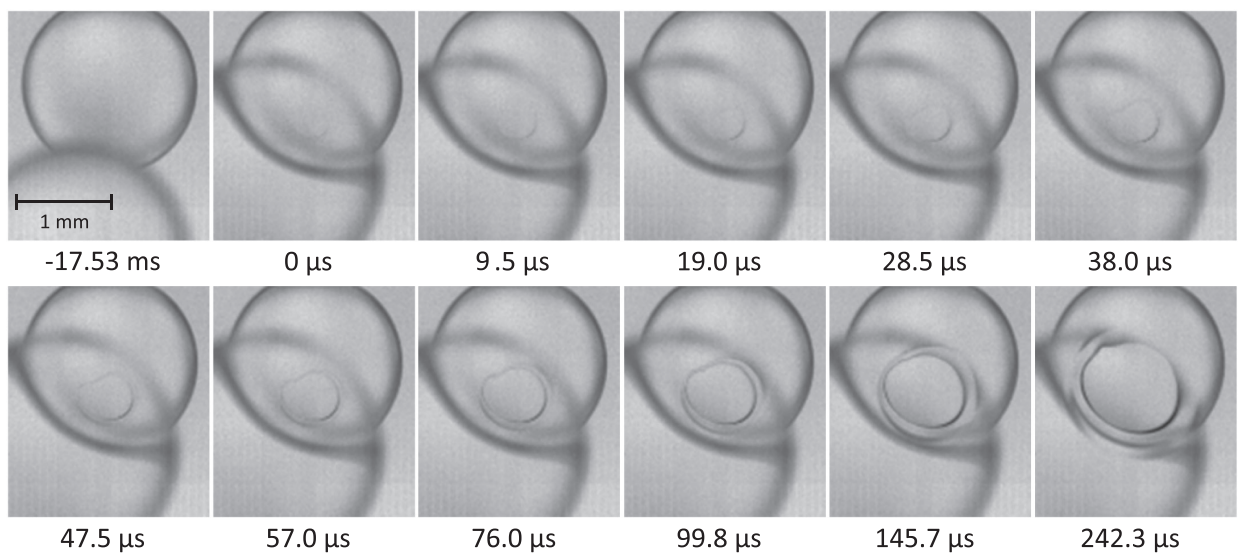

Figure 4: High-speed images of film rupture between two toluene drops in water recorded with Vision Research Phantom v711 at a framerate of $210,526 \mathrm{fps}$.

The rupture is viewed through the droplet entering from the bottom left, which optically magnifies the film. It can be seen that the film is pushed out to the top left during film rupture for about $100 \mu \mathrm{s}$.

Defining an end of coalescence for this purpose is inaccurate due to the abating oscillations.

IVb. Agglomeration: If the contact time $t_{\text {contact }}$ exceeds the drainage time $t_{\text {drainage }}$ but the critical distance $h_{\text {crit }}$ is not reached by the film drainage $\left(h_{\min }>h_{\text {crit }}\right)$, the drops move apart returning into mostly spherical shape after the maximal deformation driven by the interfacial tension. Additionally, within the distance of some tens of nanometers, the disjoining pressure components - repulsive electrostatic force (Smoluchowski 1918) and attractive van der Waals force (Hamaker 1937) - gain significant influence. If outer flow patterns do not drift the droplets apart, a stable film is formed due to a local energy minimum in attractive and repulsive forces (de Vries 1958c, Sheludko 1967) and the droplets agglomerate according to the Derjaguin-Landau-Verwey-Overbeek (DLVO) theory (Derjaguin and Landau 1941, Verwey and Overbeek 1948; see also Section 2.3 for an explanation of the DLVO theory).

IVc. Repulsion: If the necessary film drainage time $t_{\text {drainage }}$ is greater than the actual contact time $t_{\text {contact }}$ of the two drops, the film has not enough time to drain sufficiently and the collision ends in a repulsion of the droplets.

\subsection{Influencing factors}

The influencing factors on coalescence are numerous, and it is difficult to discuss them separately as several physical aspects are altered simultaneously when one property is changed. Like in many fields of physics and engineering, attempts were made to reduce the complexity of processes and obtain transferability by means of dimensional analysis. In consequence, the physical effects are reflected in corresponding dimensionless numbers and fundamental aspects such as fluid dynamics of coalescence can be discussed based on dimensionless groups. Nevertheless, the entire coalescence process is highly complex and interactions in and between multiple orders of magnitude in spatial and temporal resolution are involved, which prohibits simple correlations. Relevant dimensionless numbers in dynamic multiphase flows are the Reynolds number $(R e=\varrho v R / \mu)$, Weber number $\left(W e=\varrho v^{2} R / \gamma\right)$, Capillary number $(\mathrm{Ca}=\mu v / \gamma=W e / R e)$, Bond or Eötvös number $\left(B o=E O=\Delta \varrho g R^{2} / \gamma\right)$, Morton number $\left(M o=g \mu^{4} \Delta \varrho /\left(\varrho^{2} \gamma^{3}\right)\right)$, Ohnesorge number $(\mathrm{Oh}=\mu / \sqrt{\varrho \gamma R})$, and Marangoni number $M a=-\frac{d \gamma}{d x} \frac{\Delta x}{\mu v}$, in which $d \gamma / d x$ is the interfacial tension gradient caused by a measure $x$ (e.g. temperature, concentration, or surface concentration). One significant dimensionless quantity is the Capillary number $\mathrm{C} a$ which relates characteristic viscous (and pressure) forces to capillary forces (induced by interfacial tension; Leal 2007). Together with the dimensionless van der Waals attraction $F_{\gamma}=A_{h} /\left(\gamma R^{2}\right)$, Leal (2004) and coworkers were able to correlate several aspects in drop-drop coalescence at low velocities $(\operatorname{Re} \approx 1)$. Apart from that, the Ohnesorge number Oh was used to describe the deterministic parts (drop confluence and partial coalescence) of drop-flat interface coalescence (Kavehpour 2015; see also Section 3.1).

In general, coalescence is inherently determined by interfacial properties and already small amounts of surface-active impurities can change the coalescence probability dramatically but may have only minor or not quantifiable influence on interfacial tension. 


\begin{tabular}{|c|c|c|}
\hline $\begin{array}{c}\text { Continuous phase } \\
\text { Viscosity } \\
\text { Density }\end{array}$ & Interface & Disperse phase \\
$\begin{array}{c}\text { Energy input/velocity } \\
\text { Ilow pattern/regime } \\
\text { lonic strength, pH (if } \\
\text { aqueous) }\end{array}$ & $\begin{array}{c}\text { Surface potential } \\
\text { Surface active } \\
\text { components: surfactants, } \\
\text { particles, ions } \\
\text { Mass transfer }\end{array}$ & $\begin{array}{c}\text { Viscosity } \\
\text { Density } \\
\text { Drop diameter } \\
\text { lonic strength, pH (if } \\
\text { aqueous) }\end{array}$ \\
\hline \multicolumn{2}{|c|}{ Ambient and system conditions } \\
\hline \multicolumn{2}{|c|}{ Temperature, pressure, geometry, surface wetting, } \\
electrostatic field, microwaves, ultrasound
\end{tabular}

Figure 5: Influencing factors on the coalescence process.

Consequently, the reproducibility of experiments is a challenging task (Berger 1986, Pfennig and Schwerin 1998, Leal 2004, Soika and Pfennig 2005, Müller et al. 2008, Wegener et al. 2014) and it is crucial to be aware of the influencing factors on coalescence.

Although it is difficult to evaluate the influencing parameters separately, the main influences of the numerous properties and system conditions are discussed in the following. For this discussion, first, other parameters are virtually kept constant and the corresponding influence on the steps of coalescence is explained qualitatively. Second, important dependencies on dimensionless quantities are addressed. As coalescence is an interfacial phenomenon, it is strongly influenced by the properties and effects of continuous and disperse phases and especially the corresponding interface between them. Additionally, environmental conditions may have an impact on coalescence. Hence, the following influencing factors are classified by the properties of the continuous and disperse phases, effects at the interface, and ambient and system conditions (see Figure 5). A discussion of several influencing factors can also be found in the works of Jeffreys and Davies (1971) and Shalhoub (1975).

\subsubsection{Continuous phase}

An increase of continuous phase viscosity slows down the film drainage and thus delays or hinders coalescence (Jeffreys and Davies 1971). The viscosity is regarded in several dimensionless numbers to account for viscous effects. However, care has to be taken if continuous phase, disperse phase, or some kind of mean viscosity has to be regarded. The viscosity ratio $\mu^{*}=\mu_{d} / \mu_{c}$ determines the impact of the phase viscosities and the regarding use of dimensionless groups (e.g. selection of correlation of drag coefficient and the formation of internal flow patterns in drops; Wegener et al. 2014). Yoon et al. (2005) found a shift of the coalescence point of two drops in simple shear flow $(\operatorname{Re}<1)$ for higher viscosity ratios. Above a characteristic $\mu^{*}$, the drops coalesced after the external flow begun to pull the drops apart.

An increased continuous phase density also slows down film drainage due to the fact that a heavier fluid has a lower drainage velocity assuming hydrodynamic pressure $\frac{\varrho}{2} v^{2}$ and constant driving pressure gradient. If gravity settling is predominant, an increased density leads to higher buoyancy force, which decreases the interaction forces of droplets, decelerates film drainage, but also reduces the deformation and flattening of the interface (for creaming vice versa - depending on the density difference). Jeffreys and Davies (1971) reported that these two effects tend to cancel each other out. However, the dimensionless Bond or Eötvös number, which relate buoyancy forces and interfacial tension forces, is a possible approach to address this dependency.

The energy input in a system determines the flow pattern/regime within the system and correspondingly the relative collision velocity between disperse drops (see Table 1). A higher relative velocity decreases the contact time of two drops and consequently diminishes the chance that the film drains until film rupture (Coulaloglou and Tavlarides 1977). Additionally, a higher collision velocity leads to stronger deformations, a bigger film drainage area, and therefore an increased drainage time. Anyhow, some works propose the opposite: increased coalescence probability with higher velocity, as the authors assume that the interfacial energy has to be overcome by kinetic energy (Howarth 1964, Sovova 1981). A further discussion concerning these contradictory concepts can be found in Section 4.2. A clear experimental evidence for the dependency on relative velocity has not been found yet, as this influence cannot be decoupled from others easily. In general, the flow regime is determined by the Reynolds number, which relates the inertial forces to viscous forces. At low Reynolds numbers ( $R e \approx 1$ ), the group of Leal (Yang et al. 2001, Leal 2004, Borrell and Leal 2008, Hsu et al. 2008) was able to determine critical Capillary numbers below which coalescence occurred and above repulsion was observed. Although several fundamental dependencies on dimensionless quantities (mainly $\mathrm{C} a$ and $\mu^{*}$ ) could be identified for different physical systems, a simple scaling approach to modeling of coalescence was flawed (Leal 2004). Additionally, these findings are only valid for flow regimes in which viscous forces are dominant $(\operatorname{Re}<1)$ and drops remain in spherical shape $C a \ll 1$. 


\subsubsection{Interface}

Coalescence is an interfacial phenomenon, as it is driven by the minimization of interfacial area/energy. Consequently, factors influencing the interface have the most significant impact on coalescence.

An increased interfacial tension decreases the deformation of the drops so that the area of film drainage becomes smaller and the film drainage time is diminished, although a higher interfacial tension also decreases the surface mobility, which tends to inhibit the drainage flow and enlarges the drainage time. In general, the effect of a smaller drainage area has a stronger impact and coalescence probability increases with higher interfacial tension (Jeffreys and Davies 1971). In gentle collisions $(R e<1)$, the film radius and drainage time was found to scale with the Capillary number (Leal 2004). The interplay of viscous stresses to capillary pressure is depicted in the Capillary number. If inertial forces can be neglected, drops remain in approximately spherical shape for $\mathrm{Ca} \ll 1$ because surface tension effects dominate and already small deformations are sufficient to counterbalance pressure or stress forces on the drop surface. In contrast, for $C a \gg 1$ pressure and stress variations can only be balanced by large drop deformations.

The surface potential of oil/water interfaces in disperse systems induces a repulsive electrostatic force, which is again counteracted by attractive van der Waals forces. This interaction is commonly known as the DLVO theory (Derjaguin and Landau 1941, Verwey and Overbeek 1948). Unfortunately, the potential difference between two phases cannot be measured directly (galvanic cell), but the difference of Galvani potential difference between two systems can be measured (Watanabe 1984, Pfennig and Schwerin 1998, Pfennig et al. 1998) or indirect methods as zeta potential measurements can be applied to estimate the surface potential (Delgado et al. 2007). In general, the higher the surface/zeta potential the stronger is the repulsion between the droplets; consequently, coalescence is hindered (Rambhau 1978). However, the electrostatic force is significantly influenced by the presence of ions near the interface as a double layer of ions is formed in the liquid phases induced by the surface potential (Cheesman and King 1940, Carroll 1976, Lyklema 2000). Thus, the ion concentration and $\mathrm{pH}$ value in the investigated system determine a possible coalescence inhibition due to repulsive electrostatic effects. As these interactions are complex, no general influence can be stated here. The electrostatic effects change with the dispersion condition (oil-in-water or water-in-oil; Chen et al. 1998) and depend on ion-specific effects, which is known as the Hofmeister effect (Franks et al. 2005). In general, the influences in liquid/liquid systems are distinct from interactions of gas bubbles in liquids (Bommaganti et al. 2009). As a rule of thumb, the electrostatic repulsion is low for low ionic strength, because no double layer can be formed, and high ionic strength, because the surface potential is dampened against the surrounding by a diffusive ion layer (Pfennig and Schwerin 1998). The dampening effect is higher for multivalent ions (Watanabe 1984, Derjaguin et al. 1987); thus, these are more effective to counterbalance an electrostatic coalescence inhibition, which is commonly referred to as the SchulzeHardy rule (Schramm 2005). Furthermore, an increase of the $\mathrm{pH}$ value of the aqueous phase results in higher zeta potential due to the adsorption of $\mathrm{OH}^{-}$at the interface (Marinova et al. 1996, Creux et al. 2009) and therefore in a coalescence inhibition (Tobin and Ramkrishna 1992, Gäbler et al. 2006, Kamp and Kraume 2015). However, the electrostatic effects appear to be nearly independent from the type of oil and surface potential measurements of one specific oil can be adopted universally (Marinova et al. 1996, Creux et al. 2009).

Other surface-active components that aggregate at the interface generally reduce the coalescence probability. Amphiphilic molecules (surfactants) generally decrease the interfacial tension and delay or even inhibit the film drainage by a reduced interfacial mobility and/or steric hindering (Hodgson and Lee 1969, Hodgson and Woods 1969, Ivanov and Kralchevsky 1997, Ivanov et al. 1999, Giribabu and Ghosh 2007). A good indicator to examine the interfacial mobility of a single drop is its rise velocity. Already small amounts of surface-active components (partially) reduce the interfacial mobility and thus increase the drop's drag coefficient and reduce its rise velocity significantly (Wegener et al. 2014). Up to now, the interfacial mobility during the interaction of two fluid particles was only validated indirectly by testing the consistency of experimental data with different hydrodynamic boundary conditions (no-slip/immobile interface, full-slip/fully mobile interface; e.g. Manica et al. 2008b).

Ionic surfactants additionally influence the surface potential (see above). Generally, an emulsion is more stable (which means coalescence is hindered) if the surfactant is soluble in the continuous phase, which is known as the Bancroft rule (Bancroft 1913). The more advanced but still qualitative hydrophilic-lipophilic balance (HLB) concept was introduced by Griffin (1949), which considers the effects of the hydrophilic and lipophilic groups of a surfactant molecule (Kabalnov 1998, Rondon et al. 2006). Anyhow, the different characteristics of surfactants can be used to deplete the stabilizing effects inter alia, which 
leads to higher coalescence probability and a breaking of the emulsion. This outcome is used extensively in crude oil processes and the used chemical mixtures are termed demulsifiers accordingly (Mikula and Munoz 2000, Kokal 2005, Pereira et al. 2011). For nonionic surfactants, the HLB or advanced concepts (Borges et al. 2009) may be used to determine the composition and concentration of demulsifier to achieve the minimal emulsion stability (at $H L B \approx 10$; Ivanov and Kralchevsky 1997, Rondon et al. 2006). Accordingly, the influence of ionic surfactants can be depleted if anionic and cationic surfactants are mixed.

Apart from surfactants, fine solid particles also can adsorb at the interface, stabilize it, and hinder coalescence (Gafonova and Yarranton 2001, Sullivan and Kilpatrick 2002). This type of emulsion is commonly known as Pickering emulsion (Pickering 1907), although the effect was first described by Ramsden (1903). This type of stabilized emulsion can be broken up if the wettability of the particles is changed by adding specific demulsifiers (Mikula and Munoz 2000). If surface-active particles are present in separation (or extraction) processes, in which the interface diminishes significantly in the coalescence region, excess particles (and other surface-active components) accumulate at the interface. This foam-like or flocculent occurrence is commonly termed crud (Ritcey 1980).

In general, surface-active components (whether surfactants or colloids) exert significant influence on the interface properties. Not only the rheology (interfacial viscosity and elasticity) and interfacial mobility may change but also dynamic adsorption and desorption or other mass transfer processes can induce Marangoni convection (Ivanov et al. 1999, Schwalbe et al. 2011), which also influences interfacial mobility (Leal 2004). Recent studies were published investigating the interfacial rheology (Erk et al. 2012, Reichert and Walker 2015) with regard to film drainage and coalescence. If interfacial elastic energy equals or exceeds the interfacial energy (e.g. by rapidly increased surface concentration of adsorbed nanoparticles during confluence), coalescence is arrested at some point of the confluence process and stable conglomerates are formed, which appear frozen in the middle of the coalescence process (Pawar et al. 2011, 2012).

The mass transfer across the interface, which is inherent in many technical applications (e.g. extraction), has a significant influence on coalescence time and probability. Several investigators (Groothuis and Zuiderweg 1960, MacKay and Mason 1963a, Jeffreys and Lawson 1965, Gourdon and Casamatta 1991, Ban et al. 2000, Chevaillier et al. 2006, Kamp and Kraume 2014) found faster coalescence if a third component was transferred from disperse to continuous phase $(\mathrm{d} \rightarrow \mathrm{c})$ and an increase in coalescence time and thus dispersion stability for an inverse mass transfer $(c \rightarrow d)$. This influence of mass transfer is principally explained by Marangoni effects (Carroll 1976, Tsouris and Tavlarides 1993, Kopriwa et al. 2012). In case of a mass transfer direction $\mathrm{d} \rightarrow \mathrm{c}$, the increasing concentration of the transferring component in the separating film locally decreases the interfacial tension in this region. Thus, an additional movement of the interface toward the rim of the drainage film is induced by higher interfacial tension at the outside, which accelerates the film drainage (Tsouris and Tavlarides 1993). Furthermore, an increase of solute concentration often increases the mutual solubility of continuous and disperse phases, which could enhance coalescence additionally (Kopriwa et al. 2012). If the mass transfer occurs in the opposite direction $(c \rightarrow d)$, the interfacial tension is higher in the film region, which creates a surface flow opposite to the film drainage and thus hinders coalescence.

The influence of Marangoni effects can be categorized by the dimensionless Marangoni number. It is defined as the ratio of dynamic interfacial tension variations (caused by, for example, temperature or surface concentration gradients) and viscous stresses. Karpitschka and Riegler (2014) found a characteristic Marangoni number as a measure for the transition between immediate coalescence and noncoalescence between sessile drops of different but completely miscible liquids.

\subsubsection{Disperse phase}

Concerning the properties of the disperse phase, the drop diameter probably is the most important influencing factor on coalescence. For finely dispersed droplets (order of magnitude: some micrometers and smaller - certainly depending on $\mathrm{Ca}$ and $\mathrm{Re}$; see discussion above), the drops stay in spherical shape, which results in a Stokes-Taylor flow regime of two approaching hard spheres without a drainage film. In this regime, coalescence time diminishes with increasing drop diameter (Dickinson et al. 1988, Ivanov and Kralchevsky 1997, Basheva et al. 1999, Ivanov et al. 1999). If the droplets reach a certain size and become deformable, a drainage film is established between the droplets. As films with larger film area require more time to drain and bigger drops tend to deform stronger, the drainage (and thus coalescence) time increases with growing drop diameter. If inertial forces can be neglected, the deformation of a drop can be estimated by the Capillary number (see discussion above). Otherwise, the interplay between several 
fluid dynamic effects has to be regarded by means of $R e$, $W e, E o$, and Mo. With a graphical correlation in terms of $R e, E o$, and $M o$, it is possible to determine the shape of a free rising drop (Clift et al. 1978). A detailed discussion of the influence of droplet diameter on coalescence time and the corresponding modeling approaches can be found in Section 4.3.

The disperse phase fraction should mainly influence the collision frequency (Liao and Lucas 2010) and not the coalescence probability directly.

The disperse phase density influences the density difference to the continuous phase, which has an influence on film drainage in gravity-driven processes (see above: continuous phase). In systems with energy input, the density influences the inertia of the droplets.

The disperse phase viscosity determines the inner movement of the drop, which influences the drag coefficient and thus the relative velocity of the droplet and the velocity boundary condition of the drainage flow. Additionally, the internal flow of the drop could influence the film drainage (Leal 2004). The relevant dimensionless measure is the viscosity ratio $\mu^{*}$ as discussed above.

\subsubsection{Ambient and system conditions}

The temperature influences nearly all physical properties, in particular, density, viscosity, and interfacial tension. Thus, the influence on the coalescence process is not straightforward. Anyhow, Jeffreys and Davies (1971) stated that the coalescence probability increases in general with increasing temperature. Apparently, an increasing temperature enhances the Brownian motion, which magnifies the surface waves responsible for film rupture, and the adsorption of coalescence-inhibiting substances at the interface is reduced (Carroll 1976). In this context, the Marangoni number describing the surface tension gradient caused by a temperature change is the dimensionless measure for thermal capillary waves. However, the temperature dependency of different surfactants varies, which could lead to complex shifts in film draining and coalescence probability (Ivanov and Kralchevsky 1997). Again, the Marangoni number is relevant considering the influence of surface concentrations and temperature on interfacial tension simultaneously.

In incompressible liquid/liquid systems, the influence of the ambient pressure is probably negligible, although it also affects the density, viscosity, and interfacial tension. The pressure dependency of coalescence in gas/liquid systems was investigated by Sagert and Quinn (1976) and
Luo et al. (1997). They found hindered coalescence with increasing pressure and explained it with an adsorption at the interface inducing an immobility of the interface (Sagert and Quinn 1976) or a decrease of surface tension and an increase of continuous phase density (Luo et al. 1997).

The geometry and environment in the considered apparatus do not only influence coalescence indirectly by the induced flow pattern but also directly by the wettability of the existent surfaces. Surfaces with a lower wetting angle for the disperse phase interact as a coalescence promoter as droplets aggregate and coalesce at the surface (Jeffreys and Davies 1971, Shalhoub 1975, Sherony et al. 1978, Wines and Brown 1997, Speth et al. 2002, Amoanu 2008, Bansal et al. 2011, Agarwal et al. 2013). Hence, meshes of hydrophobic or hydrophilic materials are used as so-called coalescers. The edges, surface roughness, and crossovers of filaments enlarge the possible adhesion surface for droplets and minimize the adhesion work (Davies and Jeffreys 1969, Carroll 1976, Agarwal et al. 2013). The combination of different materials with altering wettability shows a better separation performance of coalescer meshes (Carroll 1976, Kulkarni et al. 2012), which can be explained by the alteration of wettability at the contact points of different materials promoting coalescence additionally (Davies 1982). Furthermore, higher collision rates between attached droplets on fibers and emulsion drops in the flow are induced, which enhance the coalescence rate in the coalescers (Sherony et al. 1978, Li and Gu 2005, Frising et al. 2006).

If an electrostatic field is applied to an emulsion, coalescence may be enhanced significantly. This effect is mainly used for the separation of water-in-oil emulsions (W/O; e.g. crude oil) having a nonconductive continuous phase and applying AC, pulsed DC, or DC electric fields (Carroll 1976, Eow et al. 2001, Eow 2002, Eow and Ghadiri 2003, Noïk et al. 2006). However, two types of electrostatic-induced coalescence have to be distinguished: dipolar and electrophoretic coalescence (Noiik et al. 2006). In the case of dipolar coalescence, uncharged drops are polarized and stretched by the electrostatic field inducing a dipole (Aida et al. 2010). Oppositely charged dipole sites of droplets attract each other and coalescence is promoted. In case of electrophoretic coalescence, charged droplets travel in the electric field at different velocities depending on their size and charge, mutual collisions are increased, and droplets aggregate and coalesce at the electrode. The superposition of these two effects is called dielectrophoretic coalescence (Noïk et al. 2006). 
Electromagnetic waves (microwaves) may be used for the specific and rapid heating of an emulsion, which does not have a direct influence on coalescence but raises the temperature (which again has an influence; see above; Fang et al. 1988, Frising et al. 2006).

Dispersed droplets respond to ultrasound waves if there is a nonzero acoustic contrast between the phases. The acoustic field may be used to organize droplets into thin parallel bands by standing waves or to transport drops by pseudo-standing waves, which enhances the collision rate and thus the coalescence rate (Pangu and Feke 2004, Frising et al. 2006).

\subsection{Conclusions}

The above-discussed influencing factors on the coalescence probability are summarized qualitatively in Figure 6 by classifying these parameters into causing a coalescence promotion (+) or inhibition (-) for the case that its value is increased. Parameters with neutral or not clearly identifiable influence and parameters that can act in both ways are listed in the middle.

In the coalescence process, multiple orders of magnitude in spatial and temporal resolution are involved. The surrounding flow field as well as the film drainage influence coalescence significantly. Additionally, complex interactions on and between all levels of resolution exist. Especially the film rupture is dominated by small-scale interactions (van der Waals attractions and/or thermal fluctuations) and consistent deterministic approaches

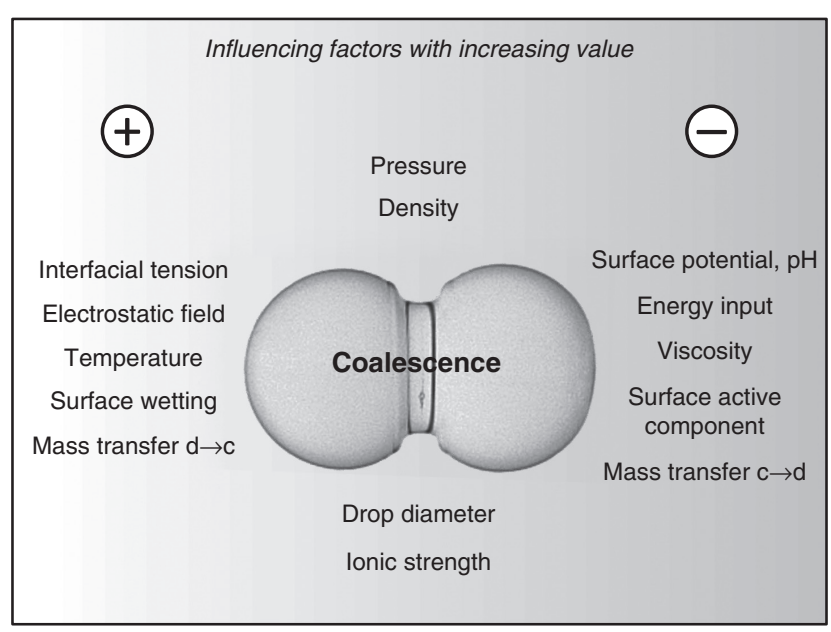

Figure 6: Influencing factors on coalescence probability categorized by causing a coalescence promotion (+) or inhibition (-) for the case that the particular value is increased. are not available up to now. Furthermore, coalescence is dominated by interfacial phenomena (e.g. adsorption of surface-active components or mass transfer effects). In the opinion of the authors, this complexity prohibits a deterministic description of the coalescence process and a straightforward reduction to dimensionless numbers and simple correlations.

The question arises if coalescence is an inherent stochastic process or, in principle, deterministic, but a clear answer cannot be given at present. If chaotic thermal fluctuations effectively dominate the film rupture, the entire process has to be considered by means of statistical methods. On the contrary, the interaction might be, in principle, deterministic, but the numerous interactions are too complex to be described properly up to now. The deterministic parts of coalescence (drop approach and collision, film drainage, and drop confluence) can be described by mechanistic approaches. However, due to the unknown film rupture event, coalescence can only be modeled by means of stochastic approaches or mean values.

\section{Experimental techniques}

This section summarizes the experimental set-ups and methods used to characterize and quantify coalescence in liquid/liquid systems. As there are numerous methods available, they are presented in increasing level of detail from simple settling tests to high-resolution film drainage investigations, as schematically depicted in Figure 7.

\subsection{Separation behavior}

In industry, the design and dimensioning of gravity separators are still based on experimental data of expensive pilot-plant tests. Therefore, many attempts have been made over the past decades to understand the separation behavior of liquid/liquid systems in small-scale settling tests and to use the results from these tests for the design of industrial separators.

As early as in 1910, Ostwald performed simple batch settling tests to investigate the separation behavior of various oil/water emulsions (Ostwald 1910). First systematic experiments with various systems of different physical properties (viscosity ratio, polarity, and interfacial tension) were carried out by Stamm and Kraemer (1925) and Meissner and Chertow (1946), although these experiments were solely of qualitative nature. Lawrence and 


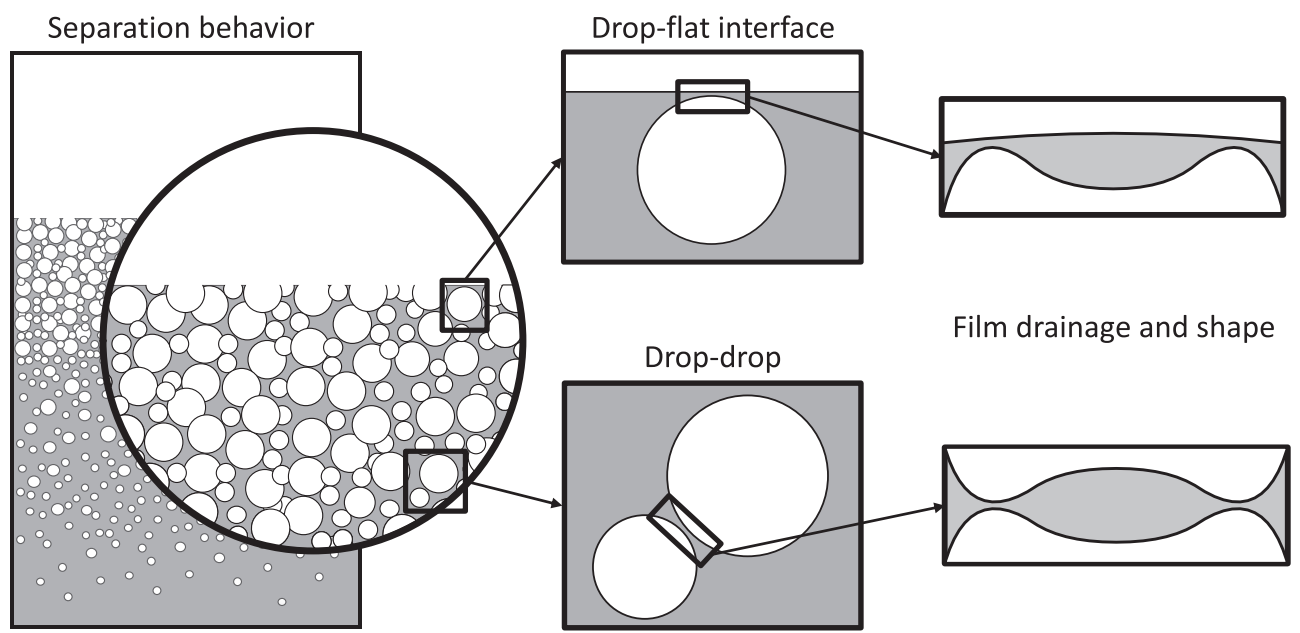

Figure 7: Methods for experimental coalescence investigations with increasing level of detail from left to right.

Mills (1954) were the first to investigate the coalescence rate by measuring the transient development of drop size distribution in different oil/water emulsions mainly to analyze the results in terms of coagulation kinetics. For the industrial design of gravity settlers, Ryon et al. (1960) and Ryon and Lowrie (1963) proposed a first empirical scale-up method based on batch and continuous settling tests by means of two uranium extraction processes. They related the height of the dispersion band, today mostly referred to as dense-packed zone, to the total specific throughput. The fundamental interactions in this dispersion band were studied by Jeffreys et al. (1970) in a continuous lab-scale settler with a kerosene/water system. They studied the dependence of the coalescence rate on the position within the dispersion band (by measuring the mean drop size with a photo camera technique) in W/O and $\mathrm{O} / \mathrm{W}$ dispersions. The drops at the coalescence front were found to be consistently larger than those at the settling front. Later, they developed a refractive index matching technique to investigate the coalescence behavior inside the dense-packed zone and obtained data for the mean drop residence times, the film thickness, and the coalescence frequency depending on the dispersion band thickness (Allak and Jeffreys 1974). Further extensive research on continuously operated horizontal gravity settlers and the separation mechanism was published by Barnea and Mizrahi (1975b,c,d,e). They described the structure of separating dispersions that form four major layers (clear continuous phase, sedimentation zone, dense-packed zone, and clear aqueous phase). The dense-packed zone consists of a dense sublayer near the coalescence front and an even concentration layer in the remaining volume. This structure of the dense-packed zone was also reported by Eckert and Gormely (1989). Concluding their experiments, they proposed an empirical correlation between batch separation time and the nominal settler capacity per unit area. The idea to correlate batch settling data and continuous settling behavior was later picked up by several authors (e.g. Golob and Modic 1977, Lee et al. 1993, and Khatri et al. 2011).

Notable in the experimental research as well as in the mathematical description of the separation of liquid/liquid dispersions is the fundamental work of the group of Hartland and Jeelani and their various coworkers (Jeelani and Hartland 1985, 1998, Hartland and Jeelani 1987, Bhardwaj and Hartland 1994, Jeelani et al. 2005a,b). In continuation of their comprehensive research on drop coalescence at interfaces, they studied several parameters influencing the separation behavior, such as the effect of the disperse phase hold-up and the dispersion height. They stated that favorably drop sedimentation and interfacial coalescence (with the continuous phase) take place during the separation process. In contrast, Barnea (1978) and Barnea and Mizrahi (1975e) reported that the controlling steps in a continuous settler are rather hindered settling and drop-drop coalescence within the dense-packed zone. In this context, Frising et al. (2006) presented experimental results for the development of drop size distributions during the separation of three different crude oil/water $+\mathrm{NaCl}$ systems. They observed that both predominantly sedimentation and coalescence-based systems exist depending on the chemical composition. In a later publication, the same authors presented a method to experimentally distinguish the contributions of sedimentation and coalescence to phase separation (Frising et al. 2008). They compared the separation velocities of a sedimentation system (coalescence hindered by high surfactant concentration) and a 
sedimentation-coalescence system. They observed that both mechanisms occur during phase separation with coalescence taking a decisive part. The influence of geometric and mixing conditions on the separation behavior in a lab-scale mixer-settler was studied by Nadiv and Semiat (1995) in a water/ $n$-heptane in paraffin oil system. The initial dispersion height and the settler diameter were found to have a significant impact on the separation time rather than the mixing conditions. The experimental data were also later used by several authors to validate their models (Henschke et al. 2002, Yu and Mao 2004). The effect of agitation time on separation behavior was also studied by Kyuchoukov and Kounev (1998). In contrast to most studies in this work, mass transfer occurs during their settling process as the experiments were carried out inside a four-stage copper extraction process.

Besides the derivation of models and design rules for industrial separators, standardized small-scale batch settling test can be a simple but helpful tool to evaluate and monitor different test systems. The stirred sedimentation cell developed by Henschke (1995) was later used to derive a model (see Section 4.1) and since has been used for various physical systems and research activities (Pfennig and Schwerin 1998, Schlieper et al. 2004, Soika and Pfennig 2005). More recently, Villwock et al. (2014a) carried out batch settling tests at two different research labs. Applying a standardization of set-ups and operation procedure, they were able to validate experimental results and to ensure that obtained data were comparable.

\subsection{Drop-flat interface coalescence}

The coalescence of a droplet with a flat interface is the limiting case of drop collision with one droplet of infinite radius. As the flat interface is easy to produce and the set-up is static and thus easy to observe, the dropflat interface coalescence was the basic principle for the first fundamental coalescence investigations of single droplets. These observations were not only of academic interest but the results were also essential for the understanding and design of gravity separators (Jeffreys and Davies 1971, Barnea and Mizrahi 1975b,c,d,e).

The first systematic investigations of drop and bubble rest times at a flat interface were performed by Rehbinder and Wenström (1930) and Rehbinder et al. (1930). In this early work, the adsorption and stabilizing effect of surface-active components also was investigated. Further systematic investigations of drop interface coalescence were reported by Mahajan $(1933,1934)$. Moreover, the author included the theory of an enclosed film that hinders coalescence and the discussion of viscosities and interfacial tension influencing coalescence. The standard set-up, which was developed by Cockbain and McRoberts (1953) and was used since then in numerous investigations, consists of a glass vessel filled with the investigated phases forming a planar interface between them. The drops are inserted in the other continuous phase either at the bottom or on top (depending on the density ratio) by a cannula. From there, the droplets rise or fall to the flat interface at which they rest for a certain time during the film drainage until coalescence occurs. The graphical representation of the resting time or stability of the droplets at the interface proposed by Cockbain and McRoberts (1953) is to plot the number of drops $N$ related to all droplets $N_{0}$ (in logarithmic scale), which did not coalesce in time $t$ plotted versus time. Thus, the median rest (or coalescence) time $\tau_{1 / 2}$ corresponds to the value of $N / N_{0}=0.5$. These investigations were continued by Gillespie and Rideal (1956), Watanabe and Kusui (1958), and Charles and Mason (1960a,b), investigating different oil phases and the influence of surfactants. In the following decades, numerous investigations were published on this topic (Allan et al. 1961, MacKay and Mason 1963b, Jeffreys and Hawksley 1965a, Davis and Smith 1976, Dickinson et al. 1988, Stevens et al. 1990, Kourio et al. 1994, Hool et al. 1998, Basheva et al. 1999, Mohamed-Kassim and Longmire 2004, Aryafar and Kavehpour 2006, Thoroddsen 2006, Ortiz-Duenas et al. 2010, Bozzano and Dente 2011, Malmazet et al. 2015), where the systematic investigations of Hartland (1967a,b,c) should be emphasized in particular. A review on experimentally determined rest times during the 1950s and 1960s can be found in the work of Vijayan and Ponter (1975).

A secondary droplet may be built during the confluence of a drop with the continuous phase due to the necking of the interface (see Figure 8). This secondary droplet is smaller as the mother drop, approaches the interface, and coalesces again. The observation of this stepwise coalescence (also known as partial coalescence) was described by Charles and Mason (1960a), MacKay and Mason (1963b), and Jeffreys and Hawksley (1962, 1965b). Several papers continuing research on this topic were published (Garti 1997, 1998, Aryafar and Kavehpour 2006, Blanchette and Bigioni 2006, Chen et al. 2006, Gilet et al. 2007, Gaitzsch et al. 2011a, Kavehpour 2015).

A recent review on the investigations of drop-planar interface coalescence and partial coalescence was published by Kavehpour (2015). It mainly focuses on the correlation of the deterministic parts of coalescence (the time of drop confluence after rupture and the occurrence 


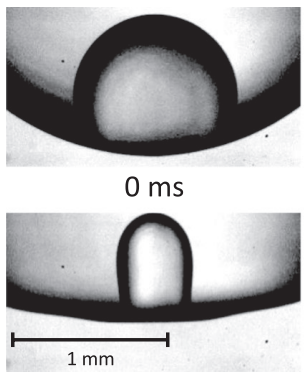

$2.50 \mathrm{~ms}$

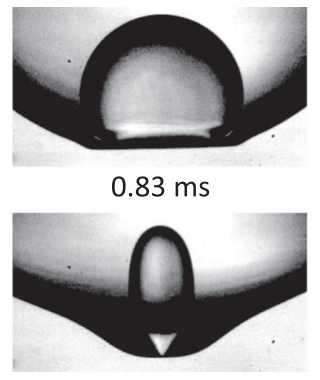

$3.62 \mathrm{~ms}$

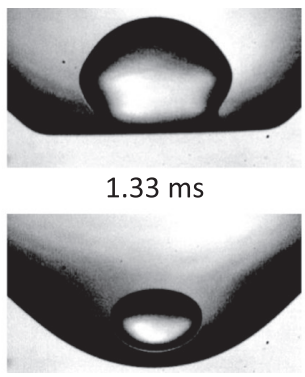

$4.67 \mathrm{~ms}$

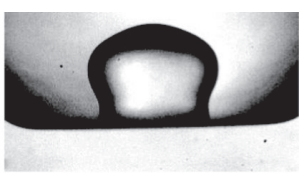

$1.67 \mathrm{~ms}$

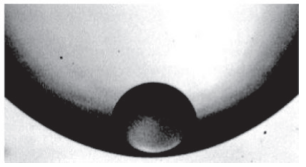

$5.83 \mathrm{~ms}$

Figure 8: Partial coalescence of a water droplet resting on the bottom of a paraffin oil drop (W/O/W double emulsion) recorded with Photron Fastcam SA-1 at a framerate of 60,000 fps (Gaitzsch et al. 2011a).

of partial coalescence) with the dimensionless Ohnesorge number $(\mathrm{O} h=\mu / \sqrt{\varrho \gamma R})$. However, various authors used the viscosity and density values of disperse and continuous phases or the specific mean values of them. Thus, care should be taken when comparing different publications.

Reynolds (1875) and Thomson and Newall (1885) used dyed drops to investigate the flow into the continuous phase after coalescence and found a vortex ring with a colored liquid trailing penetrating the phase (see also Watanabe and Kusui 1958, Charles and Mason 1960a, Aarts and Lekkerkerker 2008, Bozzano and Dente 2011). Recently, Mohamed-Kassim and Longmire (2004) and Ortiz-Duenas et al. (2010) investigated these flow patterns by particle image velocimetry (PIV).

A first detailed visualization of the film rupture by high-speed imaging was given by de Vries (1958a) observing a liquid lamella in air/water foams and by Charles and Mason (1960b) investigating water/oil emulsions. Charles and Mason (1960b) found central and off-center film rupture as well as simultaneous rupture at different locations, which was confirmed by several researchers (Allan et al. 1961, MacKay and Mason 1963b, Hartland 1967c, Aarts et al. 2005, Aryafar and Kavehpour 2006, Zdravkov et al. 2006).

The use of high-speed imaging is a powerful tool to get insights in the highly spatially and temporally resolved coalescence process (Thoroddsen et al. 2008). In early investigations, analogue film cameras were used, which means that, for every recorded sequence, a film roll of $100 \mathrm{ft}(\sim 30 \mathrm{~m})$ had to be exposed (Charles and Mason 1960b, Scheele and Leng 1971) whether the desired sequence was triggered successfully or not. Today, digital high-speed imaging offers significant advantages: nearly unlimited repetitions can be performed, which are essential for the statistical analysis of the stochastic coalescence event, sophisticated trigger methods (synchronization of experimental set-up with camera, image-based/ threshold auto-trigger) reduce the number of fail shots, the sequence can be viewed directly, and automatic picture analysis offers powerful diagnostic techniques (Ballard and Brown 1982, Gonzalez et al. 2004, Thoroddsen et al. 2008). Although analogue high-speed cameras were able to record with 3500-5000 frames per second (fps; Charles and Mason 1960a, Scheele and Leng 1971), up-to-date digital high-speed cameras grab more than 1 million fps by reducing the image resolution (i.e. $\sim 1$ million fps for Vision Research Phantom v2512 at a resolution of $128 \times 32$ pixels, Vision Research Phantom v711 at $128 \times 16$ pixels, Photron Fastcam SA-Z at $256 \times 24$ pixels, and Photron Fastcam SA-X2 at $128 \times 8$ pixels). Additionally, modern light-emitting diode (LED) illumination decreases the thermal energy input in the investigated system, which is significant for classic halogen lamps, and can be triggered and synchronized with the camera recording (Kamp and Kraume 2014).

The coalescence in three-phase systems (aqueous glycerol/paraffin oil/silicone oil) was investigated by Hartland and Robinson (1971), who also published impressive photographs of three-phase coalescence events.

\subsection{Drop-drop coalescence}

Investigations of coalescence between two drops with defined diameters were conducted with a broad variety of experimental set-ups, which can be distinguished into approaches investigating static drop contacts or dynamic drop collisions.

\subsubsection{Static conditions}

Static set-ups offer the advantage of good observability and adjustability of drop sizes. With these experiments, 
the fundamentals of film drainage and liquid bridge building can be observed in detail. However, they neglect the dynamic interactions with the superimposed flow field in technical dispersions. Most of the static set-ups are based on droplets fixed on needles or lying on top or next to each other.

Following early studies on drop-drop interactions of water drops in air (Gorbatschew and Mustel 1935, Gorbatschew and Nikiforowa 1935, Rayleigh 1878), Derjaguin and Prokhorov (1946) were the first who studied the interactions of two organic drops (different alkanes) by interferometric microphotography. Their static set-up consisted of two aligned opposing nozzles, where the drops were formed and brought into contact in an atmosphere with a given content of vapor of the same liquid. Based on their early set-up, numerous investigations were done in the past decades on the coalescence process of liquid drops in air or surrounded by other liquids. Wu et al. (2004) and Thoroddsen et al. (2005) investigated the time evolution of bridge formation between drops of different viscosities in air by high-speed imaging (up to $10^{6} \mathrm{fps}$ ). Due to technical limitations, they were only able to observe the bridge formation starting about $20-50 \mu$ s after the initial contact. Case and Nagel (2008) and Case (2009), on the contrary, observed the early-time regime ( $\left.\tau_{\text {bridge }}<10 \mu \mathrm{s}\right)$ of the coalescence process of aqueous $\mathrm{NaCl}$ solution drops in air using an electrical method. In contrast to the aforementioned studies, they found an asymptotic regime for this early stage in which coalescence occurs at the interface between two slightly flattened hemispherical drops, whereas previous experiments and simulations indicated that drops coalesce while maintaining shapes following the scaling law $R_{\text {bridge }} \propto \tau_{\text {bridge }} 0.5$ proposed by Eggers et al. (1999).

Static experiments in liquid/liquid systems with drops on two opposing nozzles were conducted by various researchers (Klaseboer et al. 2000, Danner and Schubert 2001, Chevaillier et al. 2006, Borrell and Leal 2008, Manica et al. 2008a). Other experiments differed by the arrangement of the nozzles, as some researchers used adjacent nozzles (Sagert and Quinn 1978, Ban et al. 2000, Chen and Pu 2001, Pu and Chen 2001, Wang et al. 2009, Ata et al. 2011). The main focus in almost all these studies was on the investigation of the drainage or coalescence times for various influencing parameters.

Sagert and Quinn (1978) measured the coalescence times of equal sized $n$-hexane drops in water and different aqueous electrolyte solutions and found a marked increase with increasing ion concentration. However, Danner and Schubert (2001) did not find this behavior for high viscous paraffin oil drops for ion concentrations $\leq 10^{-3} \mathrm{~mol} / \mathrm{l}$.
Furthermore, they observed a strong decrease in coalescence times for higher ion concentration ( $1 \mathrm{~mol} / \mathrm{l})$. They also reported higher drop stability and therefore longer coalescence times with larger drop sizes. This agrees with the results of Borrell and Leal (2008) and Wang et al. (2009). For drops of different diameters, Chen and $\mathrm{Pu}$ (2001) observed a decline in coalescence time - with increasing drop radius difference in three mixed oil/water systems. Pu and Chen (2001) investigated also the same systems with inversed phases and observed a "jumping coalescence phenomenon", where two separated water drops coalesce after a certain time under microgravitation without any extra force.

Ban et al. (2000), Chevaillier et al. (2006), and Wang et al. (2009) studied the influence of mass transfer on coalescence in static set-ups. Ban et al. (2000) stated an enhancement of coalescence with mass transfer from disperse to continuous phase due to faster film drainage and deceleration with inverted mass transfer direction. The investigated physical system toluene/acetone/ water shows strong Marangoni convection during mass transfer. The same trend was found by Kamp and Kraume (2014) for two colliding drops of the same test system with a dynamic set-up and by Chevaillier et al. (2006) for a glycerol/acetone/silicon oil system. Wang et al. (2009) reported a decrease of coalescence times of water drops in oil with the addition of glycerol due to mass transfer from the disperse to the continuous phase, although the interfacial tension was decreased.

To investigate the effect of interfacial aging and temperature on coalescence, Ata et al. (2011) performed singledrop experiments with kerosene oil in water. Coalescence was reduced for the aged droplets and explained by the accumulation of polar aromatic compounds of kerosene at the interface. Up to a temperature of $65^{\circ} \mathrm{C}$, they observed an increase in coalescence rates, but at $75^{\circ} \mathrm{C}$ coalescence was completely inhibited.

In a different type of static drop-drop experiments, drops are observed lying on top of or next to each other. Sata and Harisaki (1960), Sata et al. (1964), and Danner and Schubert (2001) investigated the stability of drops (W/O and $\mathrm{O} / \mathrm{W}$ ) using different surfactants. Kumar et al. (2006) performed similar experiments to examine and model the adsorption kinetics of surfactants at the organic drop interface. The coalescence of drops in the presence of an adjacent drop on a liquid/liquid interface or double emulsions, respectively, was observed by some authors with different methods, for example, tomographic PIV (Ortiz-Duenas et al. 2010) or high-speed imaging (Bozzano and Dente 2011, 2013, Gaitzsch et al. 2011a). Verdier (1999, 2001) and Verdier and Brizard (2002) studied the influence 
of viscosity ratio on coalescence in polymer blends by recording the collision of two drops placed on a glass plate and by investigating the flow field inside and outside the drop with PIV.

\subsubsection{Dynamic conditions}

In technical application, fluid dynamics has a significant impact on the drop size distribution and hence on drop breakage and coalescence phenomena. Therefore, the fundamental research of drop interactions under dynamic conditions is of special interest. Already in 1954, the original experiments of Derjaguin and Kussakov (1939) were extended: Prokhorov (1954) investigated the coalescence of organic drops in air or vapor by observing the dynamic collision of a falling with a fixed drop at different collision angles and velocities. Using a piezoelement to cause one drop to oscillate, Danner and Schubert (2001) made the transition from static to dynamic liquid/liquid experiments. They studied the influence of mechanical stress on coalescence by varying the oscillation frequency. A similar set-up was presented by Loglio et al. (2011), which was later used by Karbaschi et al. (2014) to examine the liquid bridge formation qualitatively. Scheele and Leng (1971) were the first to study the horizontal, dynamic binary collision of moving anisole droplets with varying approach velocities and angles in a stagnant water phase. As they recorded the collisions with analogue film at $5000 \mathrm{fps}$, they were only able to analyze 23 collisions in total. They found no relation between coalescence probability and impact velocity, which is in contrast to the recent results of our group for a toluene/water system and of Doubliez (1991) and Lehr et al. (2002) for an air/water system. Our group used a somewhat different set-up than Scheele and Leng (1971) in which the collision of a pendant with a rising drop can be observed (Kamp and Kraume 2014).
Figure 9 shows 10 exemplarily sequences recorded with this set-up.

However, Scheele and Leng (1971) observed that coalescence is sensitive to drop oscillation or rather the phase of oscillation at the moment of contact. Kamp and Kraume (2014) also stated that there is no clear evidence that coalescence is influenced by the drop size or drop size ratio. They also investigated the influence of mass transfer: whereas mass transfer from disperse to continuous phase resulted in enhanced coalescence, inverted mass transfer direction completely inhibited coalescence. In a slightly enhanced set-up, Villwock et al. (2014a) investigated the coalescence behavior of toluene drops of varying sizes in aqueous electrolyte solutions with different concentrations. Eiswirth et al. (Eiswirth and Bart 2008, Eiswirth et al. 2011, Eiswirth et al. 2012) also performed experiments with two moving droplets in a stagnant continuous phase. In their set-up, the drops were produced by a continuous flow of disperse phase and detached from needles due to buoyancy and inertia. They investigated the liquid bridge building and the internal flow fields during the coalescence process experimentally and numerically. The flow field and the trajectories of colliding drops were also determined by Kim and Longmire (2009) with a dual-field PIV.

A different approach to examine the coalescence of droplets under dynamic conditions is the introduction of drops in an artificial flow field. The group of Mason (Bartok and Mason 1959, Allan and Mason 1962, MacKay and Mason 1964) investigated the behavior of drops in the laminar shear flow of a Couette cylinder under many different system conditions. The group of Leal (Hu et al. 2000, Yang et al. 2001, Ha et al. 2003, Borrell et al. 2004, Leal 2004, Yoon et al. 2005, 2007, Borrell and Leal 2008) performed extensive research of two slowly $(R e \approx 1)$ colliding drops in a 2D linear flow induced by a four-roll mill. They observed that the collision angle only had a slight

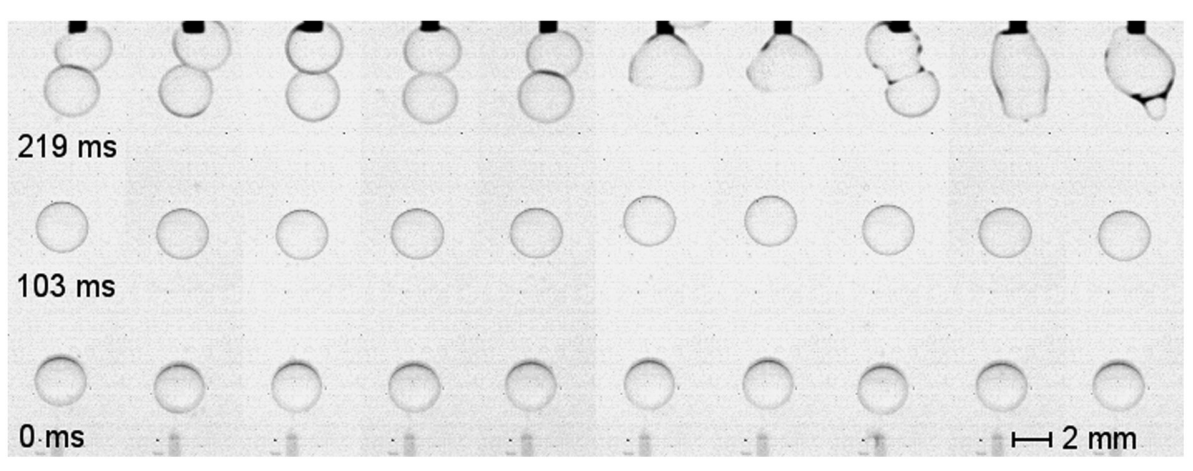

Figure 9: Ten high-speed sequences of a droplet collision in parallel (toluene/water, $d=2.5 \mathrm{~mm}$, cannula distance=20 mm, recorded with $620 \mathrm{fps}$ ). Reprinted from Kamp and Kraume (2014), with permission from Elsevier. 
influence on coalescence probability and the coalescence process was rather dominated by the time history of the force along the line of centers (Borrell et al. 2004). Also, two regimes of coalescence were observed as the drainage time was found to be dependent on the Capillary number. At high Capillary numbers, the drops were locally deformed in the film drainage region, whereas, at low Capillary numbers, the drops remained spherical (Yang et al. 2001). In addition, they reported the coalescenceinhibiting influence of surface-active copolymers $(\mathrm{Hu}$ et al. 2000, Ha et al. 2003), which was mainly explained by Marangoni effects (Yoon et al. 2007). A different approach to study collisions between two drops in shear flow was developed by Guido and Simeone (1998). In their apparatus, the shear flow is created between two parallel plates. With this technique, Chen et al. (2009) and Bruyn et al. (2013) investigated the influence of geometrical confinement on coalescence. They observed a significantly higher coalescence probability in confined conditions compared to bulk conditions.

In the more complex research of coalescence processes in double emulsions, Gaitzsch et al. (2011b) performed experiments in a counterflow cell. In the same set-up, Eckstein and Vogelpohl (1999) and Simon and Bart (2002) investigated the coalescence of a droplet swarm colliding with single droplets.

Drop interactions play also an important role in several other applications. Due to the focus of this review article on liquid/liquid systems as well as to the sheer amount of publications, we here give just a brief overview and refer to the cited literature for further reading.

There has been an extensive research on the coalescence behavior of sessile drops on rigid surfaces, as the wettability, fluid dynamics, and coalescence behavior are of special interest in the development of coalescers or separator internals in technical applications as well as in coating and printing technology (Menchaca-Rocha et al. 2001, Ristenpart et al. 2006, Hernández-Sánchez et al. 2012, Eddi et al. 2013, Karpitschka and Riegler 2014, Karpitschka et al. 2014).

Due to the great interest in the field of spray columns and combustion processes, there has also been an extensive research in dynamic interactions of liquid drops (especially water and fuel drops) surrounded by a gas phase under the influence of various physical and kinetic parameters. In particular, one should mention the works of Ashgriz (Ashgriz and Givi 1987, 1989, Ashgriz and Poo 1990), Brenn (Brenn and Frohn 1989, Brenn et al. 1997, 2001, Brenn and Kolobaric 2006), the group of Law (Jiang et al. 1992, Qian and Law 1997), and the group of Orme (Orme 1997, Willis and Orme 2000, 2003).

\subsection{Film drainage and shape}

Derjaguin and Kussakov (1939) were the first who observed the evolution of a film profile that forms a dimple in nonequilibrium state, although the term dimple was introduced later by Frankel and Mysels (1962). Over the following decades, there has been an extensive research on various aspects of film drainage. An excellent overview of experimental and theoretical approaches related to film drainage and film shape as well as the comparison of obtained results can be found in the work of Chan et al. (2011). Therefore, in the following chapter, the experimental investigations are just discussed briefly for liquid/ liquid systems.

According to Chan et al. (2011), the experimental studies can be distinguished into three major categories:

- measurements of the spatial and temporal evolution of film thickness,

- determination of time-dependent forces, and

- characterization of drop-drop interactions by measuring coalescence times.

Hartland (1967b) studied the temporal and spatial variation of film thickness beneath a drop approaching a liquid/liquid interface with photographic and capacitance measurements. They investigated rather large drops (6-10 mm) and measured average critical film rupture thicknesses in the range of several microns, where the thickness increased with drop size.

With interferometric technique - the observation of optical interference fringe patterns during the deformation of an interface - it is possible to obtain more precise data of the variations of local film thickness with time and position. This technique was first applied by Derjaguin and Kussakov (1939) and used afterwards by several researchers to study the film between two drops (Klaseboer et al. 2000, Zdravkov et al. 2003, 2006, Chevaillier et al. 2006, Manica et al. 2008b) or between a drop approaching a liquid interface (Burrill and Woods 1969, 1973a,b). Burrill and Woods (1969) used a set-up developed by Hodgson and Woods (1969) to measure the relative film thickness between toluene droplets approaching a toluene/water interface to calculate the hydrodynamic pressure distribution within the film. In the same set-up, they also investigated the film drainage process in detail for four different oil/water systems and observed that asymmetrical film drainage occurs, which was also observed by other authors (Velev et al. 1995, Klaseboer et al. 2000, Zdravkov et al.2006, Chan et al. 2011). The critical film rupture thickness was found to be in the range of 30-50 nm (Burrill and Woods 1973a). This is in good agreement with the 
experimental data of Traykov and Ivanov (1977), Manev and Angarska (2005), and Ivanov (1980) but three orders of magnitudes smaller than those obtained by Hartland (1967b). Although the critical film rupture thickness scales with the film radius or the drop size (Coons et al. 2005), the values of Hartland (1967b) seem to be a result of the limited spatial resolution of the photographic technique.

Interferometric measurements of drop-drop interactions were conducted in coalescence cells (Klaseboer et al. 2000, Zdravkov et al. 2003) in which two vertical coaxial tubes (top fixed, bottom movable) were located to form drop segments in a chamber filled with continuous phase. By moving the bottom tube at a certain approach velocity, the drops were forced to approach each other and stopped when films of specified radii were formed. For a wide range of Capillary numbers, it was observed that the drop interfaces were immobile during film thinning process; hence, film drainage was independent of the viscosity of the disperse phase (Klaseboer et al. 2000, Manica et al. 2008b). However, Zdravkov et al. (2003) observed partially mobile film drainage in the case of a slow increase of the film radius for a PEO-water/PDMS system. Chevaillier et al. (2006) studied the influence of mass transfer direction on film drainage. In the case of mass transfer from continuous to disperse phase, film drainage is slowed down, whereas, in the inverted case, it is strongly accelerated. The same influence on coalescence was also reported by other researchers (Gourdon and Casamatta 1991 for coalescence times and Kamp and Kraume 2014 for coalescence efficiency).

A slightly different set-up was established at Sofia University (Bulgaria) with the Sheludko-Exerowa cell (Sheludko 1967), a type of thin-film balance (TFB; Tabor et al. 2014), as schematically shown in Figure 10. In this experimental system, the film is formed between two deformable interfaces in a cylindrical glass tube by withdrawing the liquid from the cell and the pressure in the film is measured. The film thickness is again measured with the interferometric technique. This set-up was mostly used to study foam films (Radoev et al. 1983, Manev et al. 1997, Yoon and Aksoy 1999, Manev and Nguyen 2005) but also for emulsion films (Traykov and Ivanov 1977, Velev et al. 1995). Although a large amount of literature is available around this technique, Chan et al. (2011) stated in their film drainage review that experimental data are analyzed only in terms of the Stefan-Reynolds flat film model; moreover, important experimental results have not been reported to allow comparison with other theories.

In contrast to the experiments concerning film geometry, many researchers have investigated the occurring forces between approaching pairs of different interfaces (liquid, gas, and solid particles or interfaces, respectively).
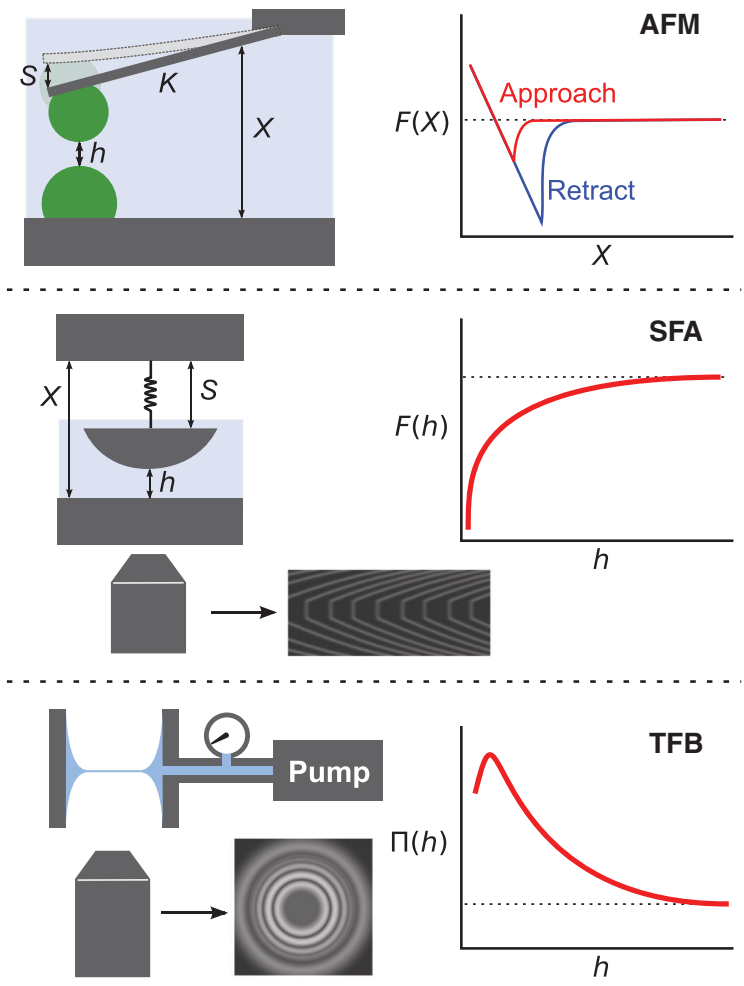

Figure 10: Overview of the most common methods for measurements of range and strength of forces between approaching interfaces (left, schematic set-up; right, typical data obtained). Reproduced from Tabor et al. (2014), with permission from the PCCP Owner Societies.

The three most common instruments - including the above-mentioned TFB - as well as the obtained data are schematically shown in Figure 10.

After the first measurements of long-range molecular forces (Overbeek and Sparnaay 1952, Derjaguin and Abrikossova 1958, Black et al. 1960), Tabor and Winterton (1969) developed the so-called surface force apparatus (SFA), where forces between surfaces could be measured for distances as low as 5-30 nm with a $3 \AA$ A distance resolution. In this set-up, the forces are determined between two atomically smooth mica surfaces across liquids or gases as one surface is held by a spring, whereas the movement of the other surface can be controlled using a piezoelectric transducer (Israelachvili et al. 2010). The corresponding distances are measured optically with multiple beam interferometry. This set-up was continuously improved and extended over the following decades mainly by the work group of Israelachvili (Israelachvili and Tabor 1972, Israelachvili and Adams 1976, Israelachvili 1987, Israelachvili and McGuiggan 1990, Israelachvili et al. 2010). With this method, Chen et al. (2004) were able to measure the surface forces and shape changes simultaneously during the approach and coalescence of two liquid/liquid and liquid/air interfaces. They 
found that droplets interact with each other at much larger distances than previously assumed. SFA was also coupled with other techniques such as X-ray scattering, infrared spectroscopy, fluorescence microscopy, and atomic force microscopy (Israelachvili et al. 2010).

Atomic force microscopy (AFM) (Binnig et al. 1986) offers several complementary advantages in terms of the material combinations and geometries, which could be explored (Tabor et al. 2012). A comprehensive overview of the AFM technique, the interpretation of obtained results, and the possible applications can be found in the work of Butt et al. (2005) and recent progress in the work of Tabor et al. (2012). AFM was initially used to measure the equilibrium forces between a solid particle fixed on a cantilever and a fluid particle immobilized on a substrate. The major difference between SFA and AFM is the large deviation in scales. The particle diameter as well as the involved forces differ by three orders of magnitude $(\mathrm{mm} / \mu \mathrm{N}$ for SFA and $\mu \mathrm{m} / \mathrm{nN}$ for AFM; Manica et al. 2008a).

In the last decades, AFM gained interest in the investigations of dynamic interactions between drops in aqueous solutions. Notable in this field is the research of Dagastine and coworkers. The first study to evaluate the feasibility of the AFM technique to probe dynamic interactions between oil droplets in aqueous solutions was published by Dagastine et al. (2004). The results were qualitative, as comprehensive modeling was still required to obtain quantitative results and fundamental understanding. The first model that takes into account the significant effects (e.g. surface forces, hydrodynamics, and drop deformation) was developed by Carnie et al. (2005) and validated with the results of Dagastine et al. (2004). In the following years, many experimental results of dynamic interactions between deformable drops in aqueous solutions (Dagastine 2006, Webber et al. 2008, Dagastine et al. 2010, Lockie et al. 2011, 2012) were published, as well as the corresponding modeling approaches, including validation with the experimental data (Manica et al. 2008b, Webber et al. 2008, Lockie et al. 2011). In addition, the AFM technique was coupled with a confocal fluorescence microscopy to get a 3D visualization of the position and separation of oil drops in complex systems where other techniques (e.g. interferometry) or theories (e.g. continuum hydrodynamics) are not applicable (Tabor et al. 2011). The extensive and detailed experiments in liquid/liquid systems were primarily carried out to gain fundamental insight into the application of AFM techniques in such systems and to evaluate the validity of common hydrodynamic and force theories.

Albeit recent progress using AFM as a technique to study dynamic interactions (structural forces, hydrodynamic drainage, and coalescence events) in liquid/liquid systems, there are still many challenges that have to be addressed in the future (e.g. specific ion effects; Tabor et al. 2012).

The third type of experimental studies on film drainage and shape is the determination of coalescence times. Although coalescence times provide less detailed information about the interaction dynamics than the previously mentioned techniques, they can give information on the stability of foams and emulsions. Experimental data for coalescence/rest times in liquid/liquid systems are available either for drops approaching a liquid/liquid interface (Charles and Mason 1960b, MacKay and Mason 1963b, Lang and Wilke 1971, Burrill and Woods 1973b, Stevens et al. 1990, Gourdon and Casamatta 1991, Basheva et al. 1999) or for drop-drop interactions (Chen and $\mathrm{Pu} 2001$, Villwock et al. 2014a). Data are mostly obtained by direct observation (e.g. high-speed imaging). Coalescence times can also be determined with interferometric technique and SFA or AFM.

One general observation is that coalescence times are increasing with increasing drop size (Charles and Mason 1960b, Lang and Wilke 1971, Burrill and Woods 1973b, Gourdon and Casamatta 1991) or the drop size ratio (Chen and Pu 2001, Villwock et al. 2014a). Concerning the physical properties of the used test system, it was observed that coalescence times are influenced by the viscosity of the disperse phase and the density difference (Lang and Wilke 1971), the polarity of the system (Stevens et al. 1990), and the interfacial tension (Gourdon and Casamatta 1991, Chen and $\mathrm{Pu}$ 2001). In the case of mass transfer, coalescence times decrease rapidly with mass transfer from continuous to disperse phase, as film drainage is improved, whereas the effect is opposite with the inverted mass transfer direction (Gourdon and Casamatta 1991). Also, the presence of surfactants or impurities can massively increase the coalescence time by changing the drainage mechanism (Burrill and Woods 1973b). In polar systems, coalescence times increase with salt addition (Stevens et al. 1990).

Besides the experimental data, numerous papers have been published concerning the modeling of film drainage, thinning, and shape during the approach of particles and the resulting forces. For further details, see Section 4.4 .

\subsection{Conclusions}

Due to the multitude of influencing factors, no standard testing conditions for coalescence are available from which the coalescence behavior of different physical 
Test system

Experimental set-up

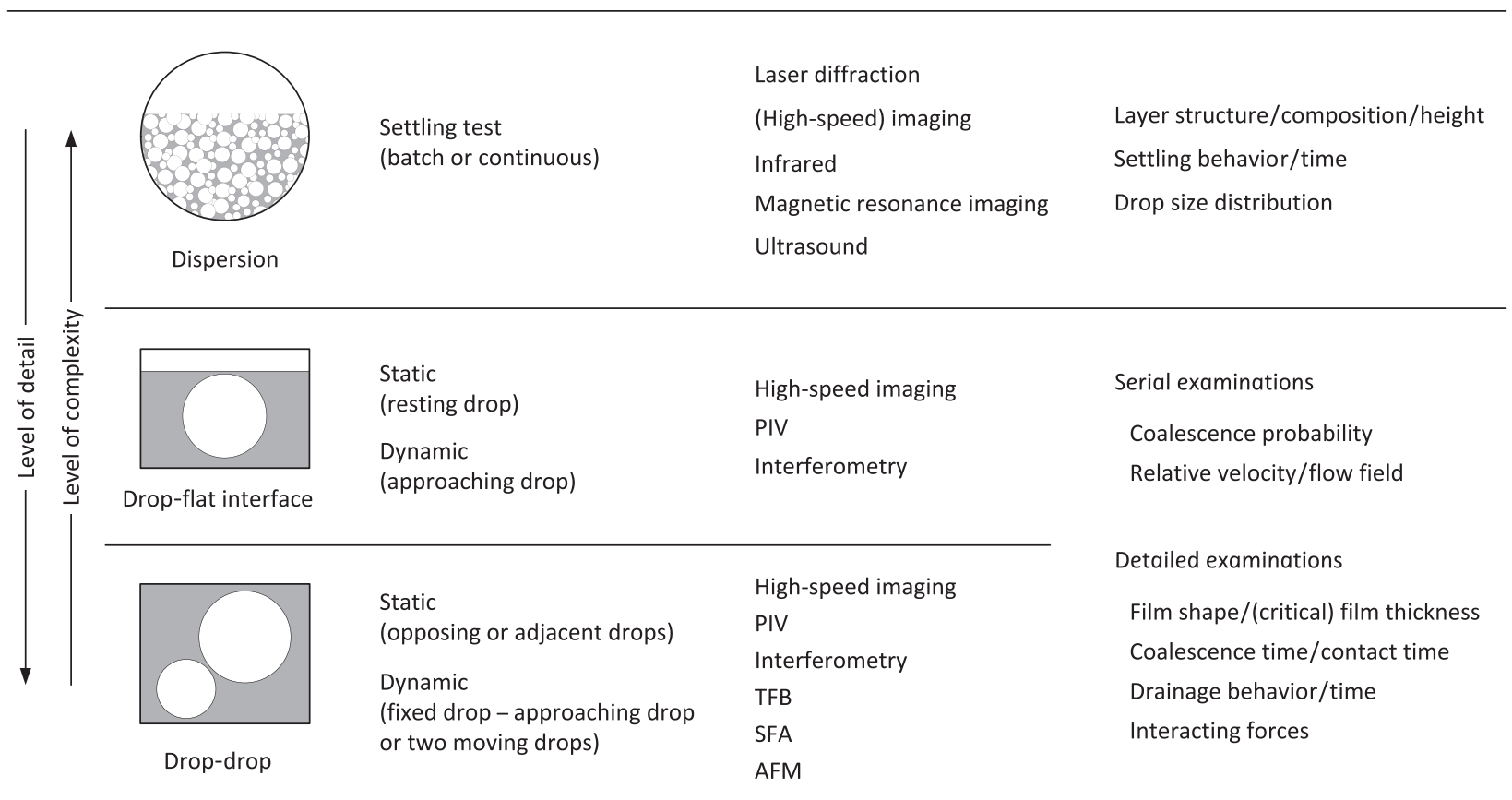

Figure 11: Summary of experimental techniques, applied measurement techniques, and obtained quantities.

systems could be deduced. Thus, several experimental set-ups are available in the literature, which were categorized and reviewed in this section. The relevant experimental set-ups are summarized in Figure 11 together with the applied measurement techniques and the obtained quantities.

Relatively simple settling tests can be performed to characterize the (static) coalescence behavior of a distinct physical system under the regarded conditions. Additionally, the transient development of the drop size distribution after an abrupt reduction of the energy dissipation rate within the system can be used to determine the (dynamic) coalescence rate indirectly (Kamp and Kraume 2015). These experiments allow a parameter fitting of coalescence models but do not permit a sound validation of those and do not allow more detailed insight in coalescence itself.

More detailed experimental techniques can be used for proper model validation and development. Using drop-flat interface coalescence investigations, already excessive research has been done up to now. First, models were developed based on these findings, but the experiments only represent the static coalescence event. The overall results are comparable to those from settling tests. To develop and validate universal coalescence models, which are also valid under dynamic conditions, the investigation of dynamic drop-drop collisions is inevitable. By performing these experiments, it is possible to reveal fundamental influencing parameters (drop size, contact angle, mass transfer, ion concentrations, $\mathrm{pH}$, surface coverage, surfactants, etc.) on coalescence probability directly and to some extent independently. Due to the stochastic nature of coalescence, serial examinations must be conducted to achieve robust and significant results. Film drainage investigations allow a direct validation of detailed drainage models, which resolve the transient development of the film shape. Furthermore, highly resolved film drainage experiments might reveal more details about film rupture and critical film thicknesses at which film rupture occurs under certain conditions.

However, all experimental set-ups investigating coalescence are a simplification of the dynamic drop collision process under real conditions. It must always be discussed if these simplifications are appropriate for the particular model and application.

In the opinion of the authors, future investigations should focus on systematic drop-drop investigations to determine the impact of influencing parameters on coalescence efficiency and rate. Based on the comprehensive experimental data available, coalescence models could be evaluated, validated, or developed further. Together with investigations in dynamic droplet swarms, the generalization of these models to a description of technical 
systems could be proven in future. Film drainage investigations are essential for the understanding of the microscopic interactions during coalescence. However, the highly resolved information gathered will not be described by large-scale modeling approaches in the near future, which are essential from an engineering point of view. At the moment, these results are rather important for academia and fundamental research than technical application.

If a proper coalescence model were available for a recent application, the ideal case would be to adapt the necessary parameter(s) with a simple and quick settling test and to be able to successfully simulate dynamic droplet swarms afterwards.

\section{Modeling approaches}

The following review of theoretical coalescence description summarizes the available modeling approaches with an ascending level of detail. First, plain models describing the settling or separation behavior of dispersions are introduced, which inherently include coalescence rate descriptions. Afterwards, an introduction to the more universal population balance equation (PBE) framework is given, which models droplet swarms of different sizes and describes the phenomena occurring between drops independently from each other. Therefore, the PBE offers a systematic bottom-up modeling for technical scale based on lab-scale experiments. Results from all kinds of investigations mentioned in Section 3 can be implemented into the submodels for drop breakage and coalescence of the PBE framework with different levels of detail (from empirical correlations to comprehensive film drainage models). For coalescence submodels, the proper description of coalescence and drainage time is important, which is discussed in the subsequent subsections. Lastly, complex numerical descriptions of fluid mechanics are presented, which offer the possibility to depict flow patterns three-dimensionally and highly detailed.

\subsection{Separation models}

All theoretical models assume that the residence time of liquid/liquid systems in large-scale horizontal gravity settlers correlates with the observed residence time in labscale batch settling tests (see Section 3.1). An extensive review on the available models for the description of separation behavior of liquid/liquid dispersions was published by Frising et al. (2006). According to them, two opposite models can be found in the literature: sedimentation-based and coalescence-based models, which will be described briefly. Modeling details such as the involved mechanisms (e.g. film drainage) and model parameters (e.g. coalescence time) can be found in Sections 4.3 and 4.4.

Sedimentation-based models were mostly proposed by the group of Hartland and Jeelani (Jeelani and Hartland 1985, Bhardwaj and Hartland 1994, Mason et al. 1995, Jeelani et al. 2005a,b). These models assume that favorably drop sedimentation and interfacial coalescence (with the continuous phase) take place during the separation process. Most experiments for the model derivations were carried out with pure liquids and rather large initial drop diameters $(>100 \mu \mathrm{m})$ and therefore presented short coalescence times, the times observed in real industrial applications (e.g. crude oil systems) are much longer due to much smaller droplets $(<10 \mu \mathrm{m})$. For that reason, sedimentation-based models have a limited range of application (Frising et al. 2006).

By considering only small, nondeformable droplets $(<100 \mu \mathrm{m})$ and taking drop-drop coalescence as the predominant mechanism into account, Lobo et al. (1993) derived a stochastical coalescence-based model. The model was tested with a hexadecane/water system and was only able to predict the experimental results qualitatively.

The model proposed by Barnea and Mizrahi (1975c,e) takes both sedimentation and coalescence into account. Another, more recent model was presented by Henschke et al. (2002). This comprehensive model takes all individual steps in the separation process (sedimentation, coalescence, deformation, and dispersion band height) into account. Their model has only one basic coalescence parameter, which can be calculated from settling experiments and is independent from the mixing conditions and phase ratios. The most recent model was reported by Noïk et al. (2013). Their model also considers sedimentation and coalescence (both types: drop-interface and dropdrop) and contains only one empirical fitting parameter that depends on the physiochemical characteristics of the system. Although the authors were able to simulate their experimental results of batch settling test with different oil/brine systems, they underline the sensitivity of their model to droplet size.

Nevertheless, as Frising et al. (2006) already stated, all available models have to be used carefully as they do not take into account the system's chemical composition and intermolecular interactions even if the empirical fitting parameters in the last two mentioned models are system specific. Also, a thorough understanding of the 
used systems is mandatory; therefore, the models should only be applied for particularly well-known systems (e.g. surfactant-free systems). In addition, common models are not applicable for systems in which mass transfer occurs (e.g. metal extraction), as they do not take Marangoni convection into account. One approach to overcome these drawbacks is the application of PBE (for details, see Section 4.2) for modeling phase separation as proposed by Grimes (2012).

\subsection{Population balance equation}

Population balance equation (PBE) is a modular framework that describes the phenomena of drop breakage and coalescence in a droplet swarm separately. For liquid/ liquid systems, numerous submodels are available (Liao and Lucas 2009, 2010) to describe the source and sink terms of droplet breakage and coalescence. These submodels can be assembled individually in the population balance, which offers the possibility of a suitable combination of submodels for each application. The effects of drop breakage and coalescence influencing the drop size distribution are decoupled from each other and detailed findings from fundamental investigations can be implemented directly. This permits to apply a bottom-up approach to describe drop size distributions in technical applications as shown in Figure 12. This fundamental

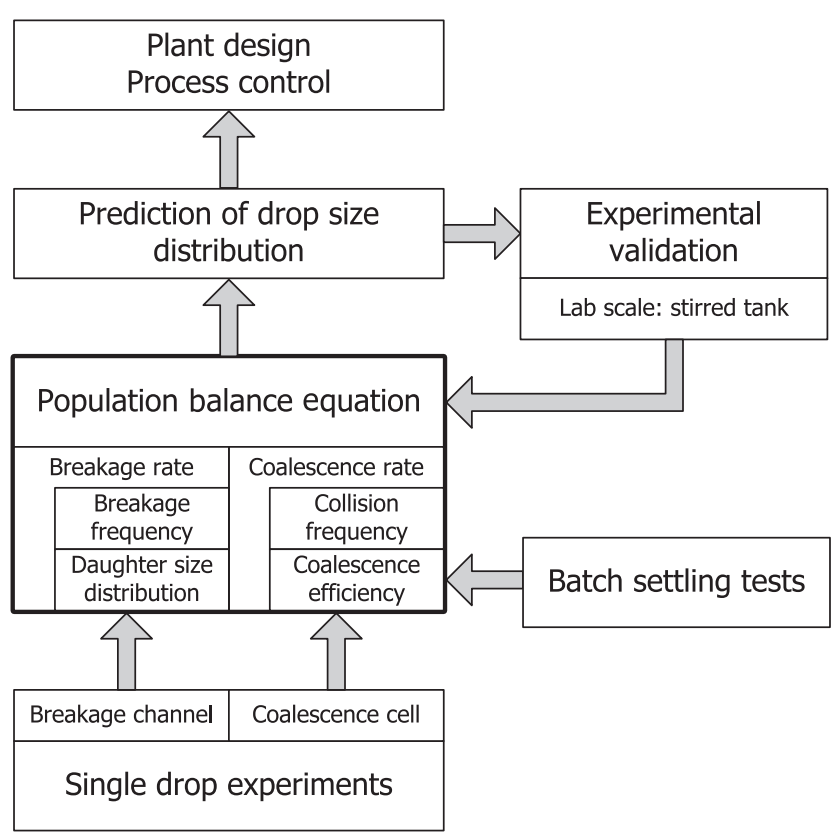

Figure 12: Bottom-up approach to predict drop size distributions from fundamental single-drop experiments (Villwock et al. 2014b). approach offers the possibility of scale-up without using empirical correlations (like, for example, as reviewed by Ghotli et al. 2013) and thus overcome geometrical dependencies and challenges during classical scale-up based on dimensional analysis and geometric similarity. Single-drop experiments and batch settling tests can be used to fit and validate the submodels for breakage and coalescence in the PBE. On this basis, a prediction of drop size distributions is possible, which can be validated in lab-scale apparatuses and be used for process control and plant design of large-scale applications (Bart et al. 2006, Müller et al. 2008, Buchbender et al. 2012, Villwock et al. 2014b). Applying this approach, the determination of breakage rate parameters from single-drop experiments was performed successfully by Maaß and Kraume (2012). Further single-drop break-up investigations in a stirred tank were performed by Solsvik and Jakobsen (2015a).

The foundation of the population balance was set by Smoluchowski $(1916,1918)$, who published the idea of balancing different particle agglomeration categories to describe the coagulation kinetics within colloid solutions. By considering death and birth terms for the distinct particle classes, a set of nonlinear differential equations was derived. In general, a population of particles can be defined by particular properties (here by material: disperse phase) and a describing variable (here: drop size). A common engineering approach is to discretize the continuous spectrum of particle sizes by distinct mesh sizes from sieve analysis and to assign a discrete number of entities in it (Ramkrishna 1985). PBE describes the time-dependent number density distribution $f\left(d_{p}, t\right)$ of particles/drops with diameter $d_{p}$ within a certain volume by birth $\dot{B}$ and death terms $\dot{D}$ for droplet breakage and coalescence and convective flows $f_{i} \dot{V}_{i}$ over the balance boundaries (Randolph and Larson 1962, Hulburt and Katz 1964, Ramkrishna 1985, 2000, Kopriwa et al. 2012, Sporleder et al. 2012, Ramkrishna and Singh 2014, Solsvik and Jakobsen 2015b):

$$
\frac{\partial f\left(d_{p}, t\right)}{\partial t}=\dot{B}_{b}-\dot{D}_{b}+\dot{B}_{c}-\dot{D}_{c}+f_{\text {in }} \cdot \dot{V}_{\text {in }}-f_{\text {out }} \cdot \dot{V}_{\text {out }}
$$

These terms are given by mechanistic submodels that, in the case of liquid/liquid PBE, account for the drop interactions depending on several factors such as continuous and disperse phase properties, interfacial phenomena, hydrodynamic characteristics, and environmental conditions (see Section 2.3). The volume-related PBE for liquid/ liquid dispersions without convective flows becomes (Valentas and Amundson 1966, Attarakih et al. 2004, Gäbler et al. 2006, Liao and Lucas 2010): 


$$
\frac{\partial f\left(d_{p}, t\right)}{\partial t}=\underbrace{\int_{d_{p}}^{d_{p, \max }} n_{d}\left(d_{p}^{\prime}\right) \cdot \beta\left(d_{p}, d_{p}^{\prime}\right) \cdot g\left(d_{p}^{\prime}\right) \cdot f\left(d_{p}^{\prime}, t\right) \mathrm{d} d_{p}^{\prime}}_{B_{b}}-\underbrace{g\left(d_{p}\right) \cdot f\left(d_{p}, t\right)}_{D_{b}}
$$$$
+\underbrace{\frac{1}{2} \int_{0}^{d_{p}} \xi\left(d_{p}^{\prime \prime}, d_{p}^{\prime}\right) \cdot \lambda\left(d_{p}^{\prime \prime}, d_{p}^{\prime}\right) \cdot f\left(d_{p}^{\prime}, t\right) \cdot f\left(d_{p}^{\prime \prime}, t\right) \mathrm{d} d_{p}^{\prime}}_{B_{c}}
$$$$
\underbrace{-f\left(d_{p}, t\right) \cdot \int_{0}^{d_{p, \max }-d_{p}} \xi\left(d_{p}, d_{p}^{\prime}\right) \cdot \lambda\left(d_{p}, d_{p}^{\prime}\right) \cdot f\left(d_{p}^{\prime}, t\right) \mathrm{d} d_{p}^{\prime}}_{D_{c}}
$$

introducing submodels for the number of daughter droplets $n_{d}\left(d_{p}\right)$, daughter drop size distribution $\beta\left(d_{p}, d_{p}^{\prime}\right)$, breakage rate $g\left(d_{p}\right)$, collision frequency $\xi\left(d_{p}, d_{p}^{\prime}\right)$ and coalescence efficiency $\lambda\left(d_{p}, d_{p}^{\prime}\right)$ and using the definition $d_{p}^{\prime \prime}=\left(d_{p}^{3}-d_{p}^{\prime 3}\right)^{1 / 3}$. As available submodels can be implemented independently from each other with different levels of detail, the modular concept of this modeling framework becomes apparent. A review on the foundation of PBEs was recently published by Solsvik and Jakobsen (2015b). For an overview of available submodels, there are extensive reviews published concerning drop breakage (Lasheras et al. 2002, Liao and Lucas 2009, Kopriwa et al. 2012, Sajjadi et al. 2013, Solsvik et al. 2013) and drop coalescence (Liao and Lucas 2010, Kopriwa et al. 2012, Sajjadi et al. 2013, Solsvik and Jakobsen 2014). Particular PBE approaches for gravity settlers were developed by Ruiz and Padilla (1996) and Grimes (2012).

However, instead of solving the PBE with discrete distributions (Valentas and Amundson 1966, Hounslow et al. 1988, Alopaeus et al. 1999, 2002, Attarakih et al. 2004, Kumar et al. 2007), there are other methods available (see Ramkrishna 2000 and Solsvik and Jakobsen 2015b for details). The calculation of the drop size distribution can be reduced to corresponding moments of the distribution, which results in faster computation time (Diemer and Olson 2002a,b, Frenklach 2002, Marchisio et al. 2003b, Attarakih 2013). A comparison of available moments methods in terms of efficiency and accuracy can be found in the work of Silva et al. (2010). Alternatively, stochastic methods such as Monte Carlo simulations can be used (Hsia and Tavlarides 1980, Goodson and Kraft 2004, Kopriwa et al. 2012), which provide more detailed information than the discrete solution as the trajectories of all droplets are known. Thus, a spatial resolution of the balanced system is possible in contrast to the other presented solution methods. Apart from that, PBE can be considered as a Boltzmann-like equation that offers the possibility of applying the corresponding statistical solution methods (Solsvik and Jakobsen 2015b).

Using the mentioned methods to solve PBE, several tools are available in the literature such as codes for proprietary software such as Matlab/Simulink ${ }^{\circledR}$ (Ward and Yu 2008, Majumder et al. 2012) as well as in-house software developments of some research groups [LLECMOD and PPBLAB (Attarakih et al. 2006, 2008, 2012) and ReDrop (Kalem et al. 2011, Buchbender et al. 2012)] and specialized software such as Parsival ${ }^{\circledR}$ (Wulkow et al. 2001).

The simultaneous solution of Navier-Stokes equations using CFD and PBEs is effective concerning computational time, as the main flow patterns of the continuous phase can be solved with CFD and the disperse drop size distribution by PBE (Hagesaether 2002, Hagesaether et al. 2000, Marchisio et al. 2003a, Drumm 2009, Drumm et al. 2009, Sajjadi et al. 2013). The coupling variables are the energy dissipation rate and phase fraction in a discrete calculation cell and the convective streams over the cell boundaries from the homogeneous CFD calculation. The latter determines the convective flows in PBE [see Equation (5)]. Energy dissipation rate and phase fraction are essential variables in most breakage and coalescence submodels. Thus, drop size distribution can be calculated for every discrete calculation cell. Implementations of coupled CFD-PBE solutions are available in Fluent ${ }^{\circledR}$ (Marchisio et al. 2003a, Sanyal et al. 2005, Drumm et al. 2009, 2010) and OpenFOAM ${ }^{\circledR}$ (Carneiro et al. 2008, Bayraktar et al. 2011, Hlawitschka 2013).

Coalescence submodels in PBE describe only the coalescence rates within a droplet swarm and do not predict single coalescence events. The collision frequency $\xi$ determines the rate of how often droplets in a system collide with each other. The coalescence efficiency $\lambda$ describes the probability that two interacting drops coalesce [see Equation (1)]. Most coalescence efficiency models describe the mechanistic parts and assume a mean critical film rupture thickness as coalescence criterion. In this manner, it is accounted for the stochastic phenomenon of coalescence and only a coalescence probability is predicted. Nevertheless, the mean coalescence rate of numerous drop collisions within a droplet swarm can be predicted in this way. There are several different modeling approaches available in the literature concerning the coalescence efficiency $\lambda$, whereas the collision frequency $\xi$ is typically described analogous to the collision of molecules in the kinetic gas theory (Chesters 1991, Liao and Lucas 2010). While the interested reader is referred to the corresponding reviews (Liao and Lucas 2010, Kopriwa et al. 2012, Solsvik and Jakobsen 2014), a short summary is given here. Apart from empirical correlations, three mechanistic modeling approaches have been established: the film drainage, the energy, and the critical approach velocity models.

The most widely used and mechanistic modeling approach is the film drainage model that was published by Coulaloglou and Tavlarides (1977). The simplified model 
relates the drainage time to the contact time (assumed as random variables):

$$
\lambda=\exp \left(-\frac{t_{\text {drainage }}}{t_{\text {contact }}}\right),
$$

based on the precondition of coalescence that the contact time of two droplets has to exceed the film drainage time. The advantage of this basic model assumption is the possibility to implement different descriptions of these characteristic times, which are numerously available in the literature especially concerning the film drainage time (see Section 4.4). Hence, various subsequent models were published mostly differing by the definition of the interface (rigid, partially, and fully deformable) resulting in different characteristic times (Chesters 1991, Tsouris and Tavlarides 1994, Liu and Li 1999, Podgórska and Baldyga 2001, Liao and Lucas 2010, Kopriwa et al. 2012).

Howarth (1964) anticipated that the adhesion forces during film drainage are weak in comparison to turbulent forces and immediate coalescence occurs if a certain relative velocity between two colliding drops is exceeded. Based on this model, Sovova (1981) proposed the energy model that assumes that the surface energy of the drops must be exceeded by kinetic energy on collision for coalescence to occur:

$$
\lambda \propto \exp \left(-\frac{E_{\sigma}}{E_{\text {kin }}}\right) .
$$

Anyhow, Sovova (1981) combined the energy model with the film drainage model of Coulaloglou and Tavlarides (1977). Simon (2004) proposed using the energy model of Sovova (1981) alone without the film drainage model.

The third modeling approach is semiempirical and simply assumes that coalescence only occurs up to a critical relative collision velocity (Lehr and Mewes 2001, Lehr et al. 2002). The originally published model equation was corrected by Liao and Lucas (2010) to

$$
\lambda=\min \left(\frac{v_{\text {crit }}}{v_{\text {rel }}}, 1\right) \text { with } v_{\text {crit }}=\sqrt{\frac{0.06 \gamma}{\varrho_{c} d_{\text {eq }}}} .
$$

This critical approach velocity model is obviously contradictive to the energy model and considers the relative velocity as the main influencing parameter of droplet coalescence.

Using the modular PBE framework, the two phenomena (droplet breakage and coalescence) can be decoupled from each other and the corresponding submodels can be validated and developed further by detailed (single-drop) investigations. In contrast to droplet swarm experiments in technical applications, the detailed investigations allow defined conditions to identify the respective influencing factors. Furthermore, a determination of possible cumulative/empiric model parameters is possible using single-drop experiments. These only need small amounts of liquid components to adapt the submodels to the present process in comparison to expensive pilot-plant tests.

However, a reliable submodel describing all influencing parameters (see Section 2.3) is not available in the literature up to now and simulations $a$ priori are not possible, as every submodel has a numerical fitting parameter in which all disregarded and eventually unknown dependencies are merged. This becomes apparent by comparing the dependencies of the influencing parameters on the coalescence efficiency $\lambda$. This is done for selected submodels in Table 2 concerning interfacial tension $\gamma$, viscosities $\mu_{c}, \mu_{d}$ and densities $\varrho_{c}, \varrho_{d}$ of continuous and disperse phases, (equivalent) drop diameter $d_{\mathrm{eq}}$, and energy input (by dissipation rate $\epsilon$, shear rate $\dot{\gamma}$, or relative velocity $v_{\text {rel }}$ ). The conversion between energy dissipation rate, shear rate, and relative velocity is discussed above (see Table 1). The dependency of the energy model from $\gamma, \varrho_{c}$ and $d_{\text {eq }}$ results from the definition of $v_{\text {crit }}$ in Equation (9) and does not apply if $v_{\text {crit }}$ is fitted to experimental data (as done by Lehr et al. 2002).

Keeping in mind that $1 \geq \exp (-x)>0$ for $0 \leq x<\infty$, all presented submodels, except the energy models in Table 2 , predict an increasing coalescence probability with increasing interfacial tension. The difference of the energy models apparently lies within the model assumption that surface energy has to vanish for coalescence. As most submodels do not consider an influence of the continuous phase viscosities and even more rarely of disperse phase, the others consider the viscosities with inconsistent proportionality. Disperse phase density $\varrho_{d}$ is only implemented by the film drainage model of Luo (1993) and the energy models and increases the coalescence efficiency with a higher value. An increasing continuous phase density $\varrho_{c}$ leads to a less probable coalescence in all models, except for the film drainage models of Tsouris and Tavlarides (1994) and Alopaeus et al. (1999). Considering the influence of drop diameter and energy input on coalescence probability, no clear trend can be found in the submodels as already discussed by Kopriwa et al. (2012). This is quite surprising as the drop diameter is one of the main measured and targeted quantities. The energy input is the most varied parameter in droplet swarm experiments (e.g. by a simple variation of stirrer speed) for which the models were developed. However, the drop diameter again depends on the energy input and is an implicit influencing parameter as it 


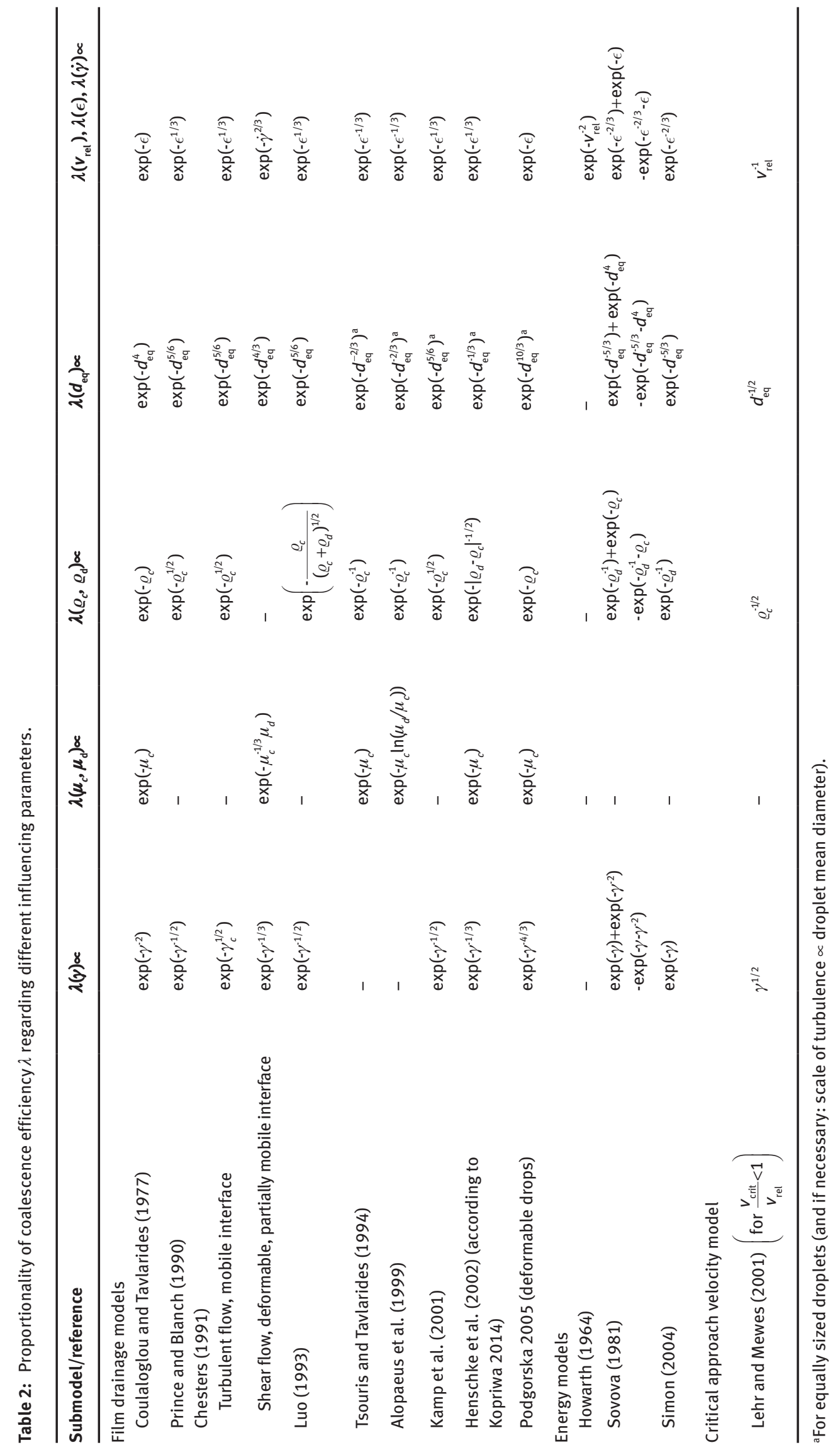


is determined by the coalescence rate. Additionally, drop size depends on the simultaneously appearing breakage rate that superimposes the dependency on coalescence efficiency additionally. Due to this coupling of breakage and coalescence, the high number of breakage models in the literature (Liao and Lucas 2009) might also correlate to the variety of coalescence models. Most models were adapted and fitted to drop size distributions of systems in which coalescence and breakage occurred simultaneously and the different influences could not be decoupled from each other. This means that an inconsistency in the breakage model could be compensated by the coalescence model and vice versa. Each submodel contains a numerical parameter that merges all nonregarded influencing parameters and allows the adjustment to experimental data. Additionally, interdependencies between these numerical parameters of coalescence and breakage models exist (Ribeiro et al. 2011). Thus, a broad variety of breakage and coalescence parameter sets can be found in the literature (Maaß 2011, Ribeiro et al. 2011). Different parameter combinations may lead to identical simulation results if the ratio between drop breakage and coalescence rate is equal, although the rates itself differ (Ribeiro et al. 2011). Instead of fitting the parameters to drop size distributions, single-drop experiments offer the possibility to decouple breakage and coalescence and allow the independent determination of the numerical parameters.

From the above discussion, it can be concluded that the coalescence probability has not been modeled comprehensively up to now. Although there are several models available in the literature, the dependency on the regarded influencing parameters is implemented diverse and in several cases even contradictory. Thus, the submodels should be selected carefully considering the general conditions under which the models were stated.

\subsection{Coalescence time}

The simplest way to describe the median resting (or coalescence) time $\tau_{1 / 2}=t_{\text {drainage }}+t_{\text {rupture }}$ of deformable droplets at a flat interface is the empirical correlation with the drop diameter by an exponent $x$ (Charles and Mason 1960b):

$$
\tau_{1 / 2} \propto d^{x} .
$$

Although the drop diameter has not the strongest influence on coalescence time, it is relatively easy to vary it solely in one physical system without changing phase properties and several other influencing factors at once. Additionally, most applications only deal with one specific physical system with mainly constant properties. Thus, many investigations were performed analyzing the influence of the drop size. A review on experimental and theoretical rest times can be found in the work of Vijayan and Ponter (1975) and an overview of experimentally determined exponents $x$ is given in Table 3. Although different liquid phases and drop sizes were investigated, the exponents were found within the range $0.6<x<3.6$, which agrees with theoretical film drainage models (see Section 4.4 for further discussion). Regarding deformable drops, the parallel discs model (MacKay and Mason 1963b) based on the Reynolds expression of the lubrication flow

Table 3: Exponent $x$ of Equation (10) for the determination of the coalescence time, $\tau_{1 / 2}$.

\begin{tabular}{|c|c|c|c|}
\hline Reference & Disperse/continuous phase & Drop diameter $d$ & Exponent $x$ \\
\hline \multirow[t]{2}{*}{ Gillespie and Rideal (1956) } & Water/benzene & $1.5-4.5 \mathrm{~mm}$ & 1.0 \\
\hline & Water/paraffin oil & $1.5-4.5 \mathrm{~mm}$ & 1.0 \\
\hline Charles and Mason (1960b) & Water/benzene & $6.5-8.0 \mathrm{~mm}$ & 3.15 \\
\hline \multirow[t]{4}{*}{ Jeffreys and Hawksley (1965a) } & Water/benzene & & $1.0(-1.3)$ \\
\hline & Water/benzene+paraffin oil & $1.9-2.8 \mathrm{~mm}$ & 0.9 \\
\hline & Water/heptane+paraffin oil & & 1.4 \\
\hline & Water/iso-octane & & 2.0 \\
\hline \multirow[t]{3}{*}{ Burrill and Woods (1973b) } & Anisole/water & $1.1-1.7 \mathrm{~mm}$ & $1.0-2.5$ \\
\hline & Cyclohexane+anisole/water & $0.8-1.3 \mathrm{~mm}$ & 0.6 \\
\hline & Cyclohexanol/water & $0.5-0.6 \mathrm{~mm}$ & $1.9-2.9$ \\
\hline \multirow[t]{2}{*}{ Basheva et al. (1999) } & Soybean oil/water+protein & $<0.1 \mathrm{~mm}$ & -1 \\
\hline & & $0.1-1.0 \mathrm{~mm}$ & 3.57 \\
\hline \multirow{3}{*}{$\begin{array}{l}\text { Gaitzsch et al. (2011a,b), } \\
\text { Gaitzsch (2014) }\end{array}$} & Water/paraffin oil & $0.15-0.9 \mathrm{~mm}$ & $0.4-2.2$ \\
\hline & & & \\
\hline & Water/silicon oil & $0.9-1.6 \mathrm{~mm}$ & 2.8 \\
\hline Thoroddsen and Takehara (2000) & Ethanol/air & $0.2-2.5 \mathrm{~mm}$ & 1.5 \\
\hline
\end{tabular}


between two parallel plates (Reynolds 1886) predicts the highest exponent:

$$
\tau_{1 / 2} \propto d^{5} .
$$

The modified cylinder model (Hodgson and Woods 1969) predicts a proportionality of

$$
\tau_{1 / 2} \propto d^{2} .
$$

For smaller rigid drops, the dependency on the droplet diameter turns to (Charles and Mason 1960b, Basheva et al. 1999):

$$
\tau_{1 / 2} \propto d^{-1} .
$$

As shown experimentally by Basheva et al. (1999) and Ivanov et al. (1999) transition from small rigid droplets to bigger deformable drops can be found for the same physical system by varying the droplet size only. Consequently, the minimal rest/coalescence time is found in the transition region between rigid sphere model (no film formation, Taylor regime) and a film drainage model (e.g. parallel discs, Reynolds regime; Kralchevsky et al. 2008) as depicted qualitatively in Figure 13.

For comparison, the experiments of Thoroddsen and Takehara (2000), who investigated the coalescence of ethanol droplets in air and found a similar exponent of $x=1.5$ as in liquid/liquid systems, are given in Table 3.

However, coalescence time obviously does not only depend on the drop diameter. Most above-mentioned models consider different phenomena and thus influencing factors to describe coalescence time. Ghosh (2004) compared different coalescence time correlations to experimental data of liquid/liquid and gas/liquid systems with diverse physical properties. Although the influencing

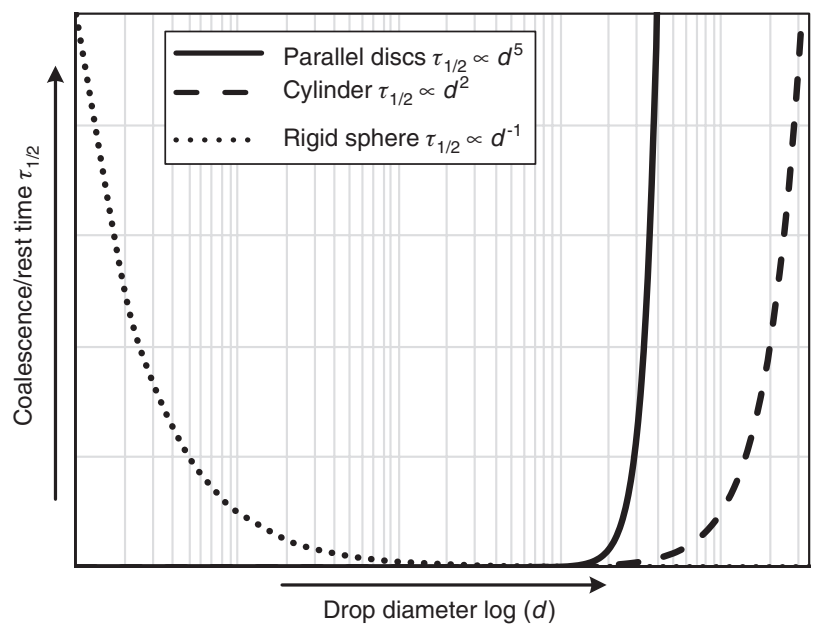

Figure 13: Rest/coalescence time $\tau_{1 / 2}$ of different modeling approaches vs. drop diameter $d$ in logarithmic scale. factors (viscosity, density, interfacial tension, and Hamaker constant) were considered additionally, substantial deviations from experimental data were found. This illustrates the limited applicability of these correlations. Therefore, many authors model the film drainage to determine coalescence time.

\subsection{Drainage models}

The drainage of the thin continuous phase film entrapped between two drops is a complex transient and 3D flow as already discussed in Section 2.2 and depicted in Figure 1. There are numerous papers and even several reviews available dealing with drainage models. Thus, only few models are discussed particularly in this work and the goal of this section is rather to give an overview over the concepts and simplifications that have been used so far to describe the film drainage.

The basic work describing film drainage was published by Derjaguin and Kussakov (1939), who also derived the radius of the draining film by balancing buoyancy and capillary pressure forces of a bubble resting at a flat interface:

$$
R_{d}=\frac{d^{2}}{4} \sqrt{\frac{2}{3} \frac{\varrho_{c}-\varrho_{d} \mid g}{\gamma}} .
$$

Additional interaction energies between deformable drops and bubbles (van der Waals, surface extension, electrostatic, steric, and depletion) were discussed and derived by Danov et al. (1993).

The link to experimental data is apparent where sophisticated direct measurements of the film shapes were performed (see Section 3.4). However, also simpler drop-interface or drop-drop coalescence investigations from which a coalescence time distribution is obtained (see Sections 3.1 and 3.3) are used to validate film drainage models. The film drainage time can be approximated by the median rest/coalescence time of the droplets: $t_{\text {drainage }} \approx \tau_{1 / 2}$ after which half of the investigated drops ( $N$ / $N_{0}=0.5$ ) coalesced if the film rupture time is neglected. In most cases, this is an appropriate assumption because generally the film rupture time tends to be one or several orders of magnitude smaller than the drainage time.

In available film drainage models, the complex film drainage was simplified concerning the following conditions:

- mobility of interface,

- drop and film shape,

- drainage flow boundary conditions, and

- (a)symmetry of film drainage. 
The interface between disperse and continuous phase can be regarded as mobile, partly mobile, tangentially immobile, or rigid, which results in different drop and film shapes as well as flow boundary conditions. High viscous drops, small droplets, or particles with a rigid interface can be modeled as spheres without film formation (Davis et al. 1989). In other cases, a film of various shapes can develop and thus be modeled. The film shape changes in time but is mostly regarded to be rotationally symmetric, which leads to a radial and temporal dependency of the film thickness $h(r, t)$. The simplest film shape is the planar and discoid film (also referred to as parallel discs) as shown in Figure 14. Depending on the pressure gradient in the film, several variations of film curvature can be found, which are called pimple, dimple (Frankel and Mysels 1962, Princen 1963), and wimple with increasing number of local extrema in the radial profile (see Figure 14). If further convolutions (minima and maxima) occur in the film, it is termed ripple (Chan et al. 2011). The most commonly observed film shape in liquid/liquid and gas/liquid systems is the dimple (see Section 3.4), which is induced by a higher pressure within the film and an outward flow. A detailed discussion of the film shapes and the corresponding modeling can be found in the work of Chan et al. (2011).

Regarding the boundary conditions of the drainage flow, all possible conditions can be found in available models: frictionless/nonviscous, slip, mobile, and (tangentially) immobile interface. Accordingly, the boundary conditions determine the velocity profile along the film thickness from plug flow to parabolic laminar profiles with and without a velocity of the interface (Lee and Hodgson 1968, Chan et al. 2011). Due to simplicity, most models assume symmetrical film drainage, although, in experiments (see Section 3.4), asymmetrical film drainage and rupture was observed. As symmetrical models predict too long drainage times, several authors proposed approaches to consider the asymmetry of film drainage (Jeffreys and Hawksley 1965b, Burrill and Woods 1969, 1973a, Jeelani and Hartland 1984, Henschke et al. 2002).

Simple film drainage models describe the decreasing film thickness $h$ over time $t$ depending on influencing parameters:

$$
-\frac{d h}{d t}=f(h, d, \varrho, \mu, \gamma, \ldots) \text { with } h \neq f(r) .
$$

These models do not provide a radial resolution of the film thickness and thus postulate planar interfaces (homogeneous film thickness) or describe only the point of minimal film thickness, which is essential for film rupture. In most cases, an analytical solution for the drainage time can be found by an integration of the model in accordance with (differential) Equation (15). The integration limits are given by the film thickness at the start of drainage $h_{0}$ down to the critical film rupture thickness $h_{\text {crit }}$ and accordingly the start of film drainage time $t_{0}$ until its end $t_{\text {drainage }}$. Together with a model of coalescence time, these drainage time models can be used directly in film drainage models of coalescence efficiency within the PBE framework (see Section 4.2).

Reviews on the extensive research and modeling of drop-drop and drop-interface coalescence in the 1950s and 1960s can be found in the works of Jeffreys and Davies (1971) and Vijayan and Ponter (1974). In the works of Chesters (1991) and Liao and Lucas (2010), drainage models for different interfacial conditions [rigid/ (partially) mobile] are summarized, which were derived analytically to obtain the film drainage times. Drainage rates considering the mentioned droplet interactions by Danov et al. (1993) are summarized by Ivanov et al. (1999), including a comparison of drainage regarding the solubility of surfactants in disperse or continuous phase.

The first drainage model was developed by Gillespie and Rideal (1956) assuming planar surfaces. It is based on the film radius calculation of Derjaguin and Kussakov (1939)

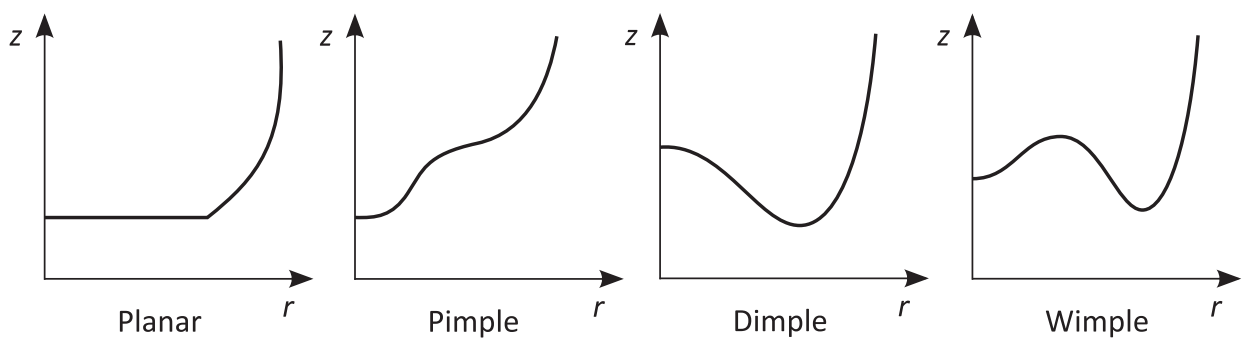

Figure 14: Rotationally symmetric (along the z-axis) shapes of interface commonly observed and modeled in film drainage: planar, pimple, dimple, and wimple.

Adapted from Chan et al. (2011) with permission of The Royal Society of Chemistry. 
[Equation (14)] and the Reynolds expression for the rate of drainage between two parallel plates (Reynolds 1886), which both were used frequently in subsequent modeling approaches. A further discussion of drainage rates, film shapes, and transition between drop-interface and dropdrop coalescence can be found in the work of Princen (1963).

Investigating thin films of foams, Scheludko (1957), Scheludko and Exerowa (1959), and Scheludko et al. (1965) revealed the fundamental aspects of disjoining pressure in drainage films with immobile interfaces covered with surfactants.

Jeelani and Windhab (2009) were able to predict coalescence times and critical film thicknesses of several published experimental investigations based on the proposed drainage model of Jeelani and Hartland (1994).

The modeling of gravity settlers is discussed in several publications (Davies et al. 1970, Jeffreys and Davies 1971, Barnea and Mizrahi 1975e) as also discussed in Section 4.1. Vohra and Hartland (1981) calculated the drainage conditions of surrounding droplets at a continuous interface to model coalescence time in the dense-packed zone of separators. Henschke et al. (2002) introduced an asymmetrical film drainage model that allows the scale-up from simple batch settling tests to the design of horizontal separators. Applying this asymmetrical drainage model for different disperse systems, Kopriwa (2014) was able to predict coalescence rates in different flow regimes in an extraction column based on settling experiments. After fitting the hydrodynamic parameters of coalescence models, a single parameter could be fitted to settling tests, which accounts for specific influences of the physical system on coalescence efficiency. Using this set of parameters, the drop size distribution within an extraction column and in the dense-packed zone of droplets was described successfully for varying physical systems (water continuous phase with different disperse organic phases: methyl isobutyl ketone, butanol, butyl acetate, and toluene) and different ion additions. This promising approach shows that a proper coalescence description of technical systems is possible using an identical 1D model for various fluid dynamic conditions. Nevertheless, it has to be mentioned that also the breakage parameters had to be fitted again to the different physical systems. Due to the coupling of breakage and coalescence, the adapted breakage parameters could also have compensated undescribed coalescence phenomena as discussed in Section 4.2.

In several other works, further 1D drainage models were proposed (Hartland and Robinson 1973, Svendsen and Luo 1996, Hsu et al. 2008, Bozzano and Dente 2011).

More detailed models include the radial shape of the draining film, but already these 2D models result in complex differential equations that have to be solved iteratively (Chan et al. 2011). Thus, an implementation in modeling frameworks such as PBE is computationally intensive or even impossible.

A detailed insight in film drainage between deformable drops and bubbles is provided in the review of Chan et al. (2011). Additionally, the authors combined the hydrodynamic flow in Stokes regime (low Reynolds number) with the interfacial deformations described by Young-Laplace equation and obtained good agreement with transient film shape measurements in different physical systems. An early detailed symmetrical model was published by Hodgson, Lee, and Woods (Lee and Hodgson 1968, Hodgson and Lee 1969, Hodgson and Woods 1969), who additionally discussed the influence of surfactants on interfacial mobility and the resulting film shapes.

A discussion of the influence of van der Waals force on film rupture can be found in the work of Abid and Chesters (1994). Jones and Wilson (1978) modeled the increased drainage speed between a drop and an interface due to circulations in the disperse phase.

A model including the effect of mass transfer on coalescence was included in drainage models by Saboni et al. (2002). Already small concentration differences lead to a decreased calculated drainage time for mass transfer from disperse to continuous phase $(d \rightarrow c)$ and longer drainage time for the reversed transfer direction, which agrees with experimental studies (see Section 2.3).

Although these and other complex models (Jeffreys and Hawksley 1965b, Burrill and Woods 1969, 1973a, Chesters and Hofman 1982, Chen 1985, Hartland et al. 1994, Li 1994, Saboni et al. 1995, Jeelani and Hartland 1998, Bazhlekov et al. 2000, Hagesaether et al. 1999, 2000, Klaseboer et al. 2000, Yeo et al. 2003, Baldessari and Leal 2006, Toro-Mendoza and Petsev 2010, Zhang et al. 2010) are able to model radial profiles of the draining film, the simplification of the complex 3D flow to two dimensions $(h, r)$ is inaccurate because the film drainage is at least unsymmetrical in the moment just before film rupture. Consequently, the description of film drainage and the $a$ priori prediction of coalescence are still challenging tasks for future investigations.

\subsection{Computational fluid and particle dynamics}

The numerical description of fluid mechanics offers the possibility to depict flow patterns and characteristic dynamics in three dimensions and at high level of detail. Nevertheless, the possibilities of the available methods are mainly limited by computational time. In coalescence 
modeling, the challenge is the interaction of fluid dynamics at different levels of scale in time and space. The film drainage occurs at spatial expansions of about nanometers and time scales of microseconds to seconds. However, the collision of droplets with fully mobile interface (at which distinctive film drainage would only occur) takes place in dimensions of several micrometers to millimeters and time scales of milliseconds to seconds. Consequently, for a comprehensive simulation of coalescence, fluid mechanics would have to cover several orders of magnitude in space and time (Janssen and Anderson 2011).

In the following subsections, a brief overview of the most popular approaches is given. These are categorized by their level of detail: computational fluid dynamics (CFD), which assumes the fluid as a continuum; lattice Boltzmann methods (LBM), which are solved on the fluid particle level; and molecular dynamics (MD), which resolve molecules down to their atomic structure. For further reading, the interested reader is referred to the cited literature.

\subsubsection{Computational fluid dynamics}

Computational fluid dynamics (CFD) is (mostly) based on the Navier-Stokes equation that assumes the fluid as a continuum (Anderson 1995, Wesseling 2001). Thus, the simulation is restricted to sizes significantly larger than the fluid molecules so that their particulate character can be neglected. The describing equations are solved within discrete cells that build a mesh in the physical volume. The mesh can be static during simulation or might be recalculated and adapted after each iteration step. For multiphase simulations, a differentiation between the apparent phases has to be performed. For the analysis of coalescence, numerical methods can be roughly distinguished into two numerical methods: front capturing and front tracking techniques (Janssen and Anderson 2011). Examples are volume of fluid (VOF, Hirt and Nichols 1981), level set (Osher and Sethian 1988), and diffusive interface method (DIM; Anderson 1995) for front capturing methods and the boundary integral methods (Pozrikidis 1992) for front tracking methods. The major drawback of the front capturing methods is their incapacity to cover the wide range of length scales that are relevant during the entire coalescence process. Furthermore, the accuracy of these methods is limited by the grid size. Particularly, when the drops come in close contact, in most simulations, only the numerical merging of drops is observed as the length scale at which film rupture takes places is orders of magnitude smaller than the discretization length scale applied (Janssen and Anderson 2011). The front tracking methods or boundary integral methods have the advantage that their implementation involves integration only on the interfaces, thus resulting in higher performance (Bazhlekov et al. 2004). The disadvantages of the front tracking are mainly issues due to mathematical complexity (e.g. numerical instability caused by interfacial tension at small Capillary numbers; Zinchenko et al. 1997, Bazhlekov et al. 2004, Janssen and Anderson 2011).

Several authors studied the fluid dynamics of drop coalescence (Yiantsios and Davis 1991, Nobari et al. 1996, Loewenberg and Hinch 1997, Rother et al. 1997, Eggers et al. 1999, Duchemin et al. 2003, Hardt 2005, Yoon et al. 2007, Eiswirth et al. 2012, Mohammadi et al. 2012, Chen and Wang 2014, Mansouri et al. 2014) and were able to reproduce the specific shapes after film rupture such as the formation of coalescence bridge (Menchaca-Rocha et al. 2001, Decent et al. 2006). Due to the above-discussed restrictions, coalescence was artificially induced by "smeared" interfaces or by introduced conjunctions between the interfaces and the film drainage could not be resolved adequately. Mason et al. (2012, 2014) overcame this problem by introducing a simultaneously calculated 1D submodel that described the film drainage between the colliding droplets. This coupled multiscale approach of CFD and drainage modeling is a promising method to cope with the resolution problem in space and time discussed above. Nevertheless, the quality of the film drainage model (see Section 4.4) determines the solution of the whole simulation thoroughly.

Besides the aforementioned numerical methods, there are several other approaches to reproduce the coalescence process such as smoothed-particle hydrodynamics (Meleán and Sigalotti 2005, Acevedo-Malavé and Garcia-Sucre 2011a,b, Acevedo-Malavé 2012) or the spineflux method (Mashayek et al. 2003).

As mentioned previously, in recent years, the implementation of PBE into CFD became of higher interest (see Section 4.2). With this coupling, it should be possible to fully describe the transient drop size distribution in turbulent liquid/liquid flows. A comprehensive review on this topic can be found in the work of Sajjadi et al. (2013).

\subsubsection{Lattice Boltzmann methods}

Lattice Boltzmann methods (LBM) solve the stochastic partial differential Boltzmann equation on a discrete lattice of particle positions within the given simulation volume. The fluid is regarded to consist of particles that are represented by a distribution function. Total mass and momentum balance are solved together with specific 
particle collision models to calculate the transient change of the particle distribution function (Chen and Doolen 1998). Under certain boundary conditions, the Boltzmann equation can be transferred into the incompressible Navier-Stokes equation (Chen and Doolen 1998). Due to these characteristics, LBM can also be regarded as a mesoscopic method between CFD and MD. The advantage of LBM for multiphase simulations is the inherent possibility to introduce different particle types without a need to recalculate the interface due to their discrete nature.

Similar to the studies with CFD, several papers on LBM dealing with drop coalescence have been published in the last decade (Inamuro et al. 2004, Premnath and Abraham 2005, Jia et al. 2006, Dupuy et al. 2010, Gac and Gradon 2011, Sun et al. 2013, 2014). Particularly, the extensive research of Derksen et al. has to be mentioned (Shardt et al. 2013, 2014a,b, Komrakova et al. 2015). With their approach, they were able to obtain a critical Capillary number that determines the transition between drop repulsion and coalescence in simple shear flow (Shardt et al. 2013, 2014a). Their results were also qualitatively consistent with the experimental data of Chen et al. (2009).

\subsubsection{Molecular dynamics}

Molecular dynamics (MD) simulations offer the possibility to gain detailed insights in molecular interactions with setting only fundamental input parameters. Newton's equations of motion are solved on atomic scale by defining universal interatomic potentials (the set of parameters is commonly called force field) that induce forces between atoms and molecules (Alder and Wainwright 1959, Hoover 1986, Israelachvili 1991). Most common are force fields based on Lennard-Jones potentials (Jones 1924). MD simulations offer the possibility to simulate the microscopic processes of film drainage and rupture $a$ priori without introducing assumptions or simplifications as discussed above. However, those simulations are restricted to several thousands of atoms. Space and time are resolved only in the order of nanometers and nanoseconds.

There are several MD simulations available that investigate the coalescence of drops in vacuum (Greenspan and Heath 1991, Svanberg et al. 1998, Koplik et al. 2002, Kalweit and Drikakis 2006, Liao et al. 2007) or a gas phase (Wang et al. 2015). Kalweit and Drikakis (2006) were able to depict the different interaction regimes that occur in gas/liquid systems (coalescence, stretching separation, and scattering) by varying the collision angle between water clusters of approximately 10,000 Lennard-Jones particles. Wang et al. (2015) simulated the interaction of water nanodroplets (consisting of 3360 molecules each) with dissolved $\mathrm{KCl}$ ions in nitrogen atmosphere within an electrostatic field and were able to predict coalescence and noncoalescence according to the critical electric field strength.

The application of MD to liquid/liquid systems is computationally more expensive and only two publications are known to the authors so far. Zhao and Choi (2004) investigated clusters of 100 water molecules coalescing in $n$-heptane showing that the dynamics of the coalescence process until the formation of the coalescence bridge differs from the case in vacuum. However, the confluence of the droplets after the bridge formation was comparable in $n$-heptane and vacuum.

Rekvig and Frenkel (2007) investigated the rupture of an aqueous film between two oil layers in the presence of surfactants with the goal to extrapolate the coalescence of two oil droplets in such a system. Additionally, the authors used a mesoscopic model that is a common simplification approach in MD modeling: multiple covalently bound atoms are grouped together to single "coarsegrained" particles with adapted interaction parameters in the applied force field. Thus, the total amount of particles was reduced and two orders of magnitude were gained in length and time scale.

Generally, MD simulations offer a great potential for detailed and a priori simulations of the coalescence process but are still computationally expensive and thus restricted in space and time. Thus, the film drainage with dimple formation of two droplets (which would need to have several micrometers in diameter to have considerably mobile interfaces) will not be realizable with MD simulations in the near future. A solution might be a coarse-grained model that, on the contrary, would lose the $a$ priori nature due to inherent simplifications incorporated in the mesoscopic model.

\subsection{Conclusions}

Up to now, no uniform coalescence model is available, which can describe the coalescence process comprehensively considering all spatial and temporal scales. The presented modeling approaches to predict important characteristics of the coalescence process are summarized in Figure 15. Additionally, possible implementations of detailed submodels into the coalescence rate are indicated.

A common approach is to divide coalescence rate into collision frequency and coalescence probability. 


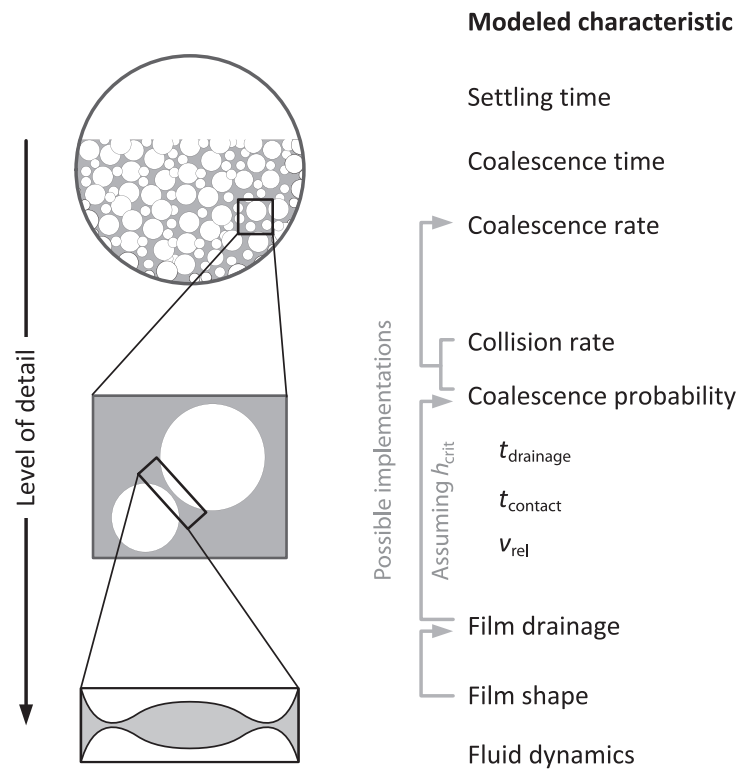

Modeling approach

(Semi) empirical correlations

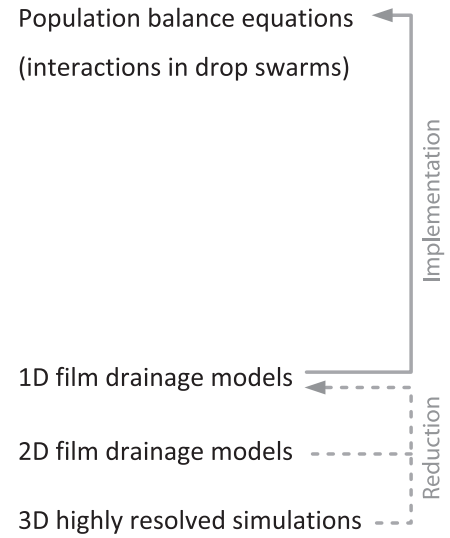

Figure 15: Summary of modeled characteristics and the corresponding modeling approaches.

In general, simple correlations of influencing factors fail for generalized modeling due to the various influencing factors and the resulting strong dependency on the regarded physical system. Although several models describe the coalescence probability properly under several restrictions and assumptions, more generalized models are necessary in the opinion of the authors. Anyhow, a determination of the needed level of modeling details for a proper coalescence description is not possible $a$ priori and has to be evaluated for each application. In most coalescence models, a consequence of simplifications and assumptions is that the nonregarded parameters and aspects are merged into numerical fitting parameters.

Due to the stochastic occurrence of coalescence, most models assume the coalescence probability as a random variable to describe the mean coalescence rate within a drop swarm. Thus, no deterministic prediction of a single coalescence event has to be made. The application of PBEs provides a good framework for these models. Most mechanistic models base on some kind of film drainage model that relates the drainage and contact times as random variables to obtain the coalescence probability: $\lambda \propto \exp \left(-t_{\text {drain }} / t_{\text {contact }}\right)$. However, it has been discussed above that the implementations of dependencies on physical parameters differ significantly (see Table 2). This shows that a well-founded validation is strongly needed, which requires proper experimental techniques (see Section 3.5). The variety of models increases additionally because any combination of models for film drainage and contact time can be made (Chesters 1991, Liao and Lucas 2010). Nevertheless, without validation, no precise answer can be given, which is the best or correct way to model the film drainage properly. Especially for "simple" 1D models that can be implemented in population balances, assumptions and simplifications are unavoidable to obtain an algebraic equation. In general, models assuming flat or symmetrical film shapes tend to overestimate the drainage time. The most crucial assumption in all drainage models is the definition of the critical film thickness $h_{\text {crit }}$ at which the film is expected to rupture and coalescence occurs. In most models, $h_{\text {crit }}$ is set to a fixed value or simple correlations are used, although this parameter has not been unveiled up to now and values varying in several orders of magnitude can be found in the literature (see Section 2.2). Additionally, only few models consider the transition in drainage regimes from film drainage to hard spheres (Taylor) with decreasing drop size.

Detailed 2D and 3D models are too complex to be implemented in large-scale simulation frameworks such as PBE, but they are important to understand the physical interactions together with experiments of film drainage.

From an engineering point of view, the authors believe that it is important to describe technical apparatuses using models that are as simple as possible but valid for extrapolation to perform a reliable scale-up of apparatuses. For this purpose, PBE offers a feasible framework with its modular structure and adaptable submodels. Therefore, a "simple" 1D mechanistic and universal coalescence submodel is essential, which describes the main influencing parameters correctly. Inevitably, numerical fitting parameters have to be determined by reference experiments. This adaption to the real physical 
system has to be kept as simple as possible to obtain practicability in industrial applications. In this regard, Kopriwa (2014) showed in a promising work that coalescence could be described in dynamic and stagnant zones within an extraction column by fitting the asymmetrical Henschke model (Henschke 1995, Henschke et al. 2002) to simple settling tests.

\section{Summary and outlook}

This review is divided in three parts. The first part describes the coalescence process in detail discussing the sequential steps during a collision of two drops that could result in coalescence, agglomeration, or repulsion. Additionally, the various parameters are discussed, which influence the coalescence probability of two drops in contact.

In the second part of this work, several experimental techniques are reviewed, which were used for coalescence investigations at different levels of detail. For example, simple batch settling tests give only a rough estimate of the coalescence behavior of the investigated liquid/liquid system, but the settling behavior can already be used for design rules of technical gravity settlers. More detailed investigations of single drops are able to study the impact of influencing parameters independently from each other regarding film drainage and coalescence probability. Sophisticated methods even reveal the film shape during drainage and the meanwhile occurring forces.

In the third part of this review, available mechanistic modeling approaches are summarized. Coalescence efficiency models based on various assumptions and levels of detail concerning drop collision and film drainage time can be implemented in the PBE framework. This framework offers the simulation of technical apparatuses based on fundamental and independent single-drop submodels and experiments. More detailed simulations such as 3D film drainage models, CFD, and MD offer the possibility to describe the coalescence process in detail but are restricted in simulation time and space due to computing time.

This review reveals that the coalescence of droplets was investigated extensively in the past decades and the specific steps during coalescence (collision, film drainage, and rupture) can be regarded to be understood in principle. Nevertheless, the interplay between interaction forces at different scales during the coalescence steps is extremely complex and not understood in detail so far. The influencing factors on coalescence are numerous and already minor changes or impurities might have severe influence. Additionally, film rupture is determined by small-scale interactions (van der Waals attraction and/ or thermal fluctuations) that are, to some extent, chaotic and extremely difficult to resolve experimentally and in simulations. Due to this complexity, the coalescence process has to be described by stochastic approaches and a prediction or simulation of single coalescence events $a$ priori is not possible up to now. Concerning the deterministic parts of coalescence, various and detailed theoretical models were developed. Recent systematic investigations based on settling tests showed that coalescence can be modeled successfully for different physical systems by 1D approaches describing several fluid dynamic conditions. Thus, in the opinion of the authors, a comprehensive and systematic approach regarding the different scales and influencing factors of coalescence might lead to the successful description of coalescence in technical applications in the near future.

As already stated by Leal (2004), coalescence investigations are still an area of active research. The authors believe that especially fundamental systematic experimental studies that investigate the influencing parameters independently are necessary to validate and develop theoretical descriptions and close the missing link between the model and the experiment. Although a deterministic prediction of the coalescence event might not be possible, the coalescence probability or rate could be anticipated by reliable models in the future.

\section{Nomenclature}

\section{Latin letters}

$A_{h} \quad$ Hamaker constant $\quad[\mathrm{N} \mathrm{m}]$

$\dot{B}_{b} \quad$ birth rate by breakage $\quad\left[\mathrm{m}^{3} / \mathrm{s}\right]$

$\dot{B}_{c} \quad$ birth rate by coalescence $\quad\left[\mathrm{m}^{3} / \mathrm{s}\right]$

Bo Bond number $\mathrm{Bo}=\Delta \varrho g R^{2} / \gamma$

$C_{D} \quad$ drag coefficient/friction factor

$C_{D, S} \quad$ single-drop friction factor

$C_{D, \varphi} \quad$ drop swarm friction factor

$\mathrm{Ca} \quad$ Capillary number $\mathrm{Ca}=\mu \mathrm{v} / \gamma$

$d_{32} \quad$ Sauter mean diameter $\quad[\mathrm{m}]$

$d_{p} \quad$ particle/droplet diameter [m]

$d_{\mathrm{p}, \max } \quad$ maximal particle/droplet diameter [m]

$d_{e q}^{p, \max } \quad$ equivalent drop diameter $\quad$ [m]

$\begin{array}{lll}d^{*} & \text { specific drop diameter } & \text { [m] }\end{array}$

$\dot{D}_{b} \quad$ death rate by breakage $\left[\mathrm{m}^{3} / \mathrm{s}\right]$

$\dot{D}_{c} \quad$ death rate by coalescence $\quad\left[\mathrm{m}^{3} / \mathrm{s}\right]$

$E_{\sigma} \quad$ surface energy [J]

$E_{k i n} \quad$ kinetic energy [J]

Eo Eötvös number $E o=\Delta \varrho g R^{2} / \gamma$

$f \quad$ number density function $\left[\mathrm{m}^{-3}\right]$ 


\section{$F$}

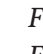

$F_{\gamma} \quad$ dimensionless van der Waals

$g \quad$ gravity acceleration

$g \quad$ breakage rate

h film thickness

$h_{0} \quad$ film thickness at the start of drainage

$h_{\text {crit }} \quad$ critical film thickness at which film rupture occurs

$h_{\min } \quad$ minimal film thickness

$M a \quad$ Marangoni number $M a=-d \gamma / d x \cdot \Delta x /(\mu v)$

Mo $\quad$ Morton number $M o=g \mu^{4} \Delta \varrho /\left(\varrho^{2} \gamma^{3}\right)$

$n_{d} \quad$ number of daughter droplets

$N \quad$ number of (coalescence) events

$N_{0} \quad$ total number of (coalescence) events

$\mathrm{Oh} \quad$ Ohnesorge number $\mathrm{Oh}=\mu / \sqrt{\mathrm{gr} R}$

$R, r \quad$ radius

$R_{\text {bridge }} \quad$ coalescence bridge radius

$R_{d} \quad$ draining film radius

$R_{e q} \quad$ equivalent droplet radius

$\operatorname{Re}=\left(v d \rho_{c}\right) / \mu_{\varphi}$

$s \quad$ drop separation distance

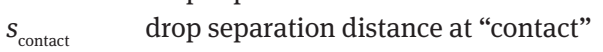

$S_{\text {interaction drop separation distance at which }}$

interaction occurs

$t_{0} \quad$ starting time

$t_{\text {drainage }} \quad$ film drainage time

$t$

$t$ coalescence

$t_{\text {rupture }}$

$v$

$v_{\text {crit }}$
$v_{\text {rel }}$

$v_{\text {rel }}$
$\dot{V}$

$\dot{V}$
$W e$

coalescence time, $t$ drop "contact" time $=t_{\text {drainage }}+t_{\text {rupture }}$

film rupture time

velocity

critical velocity

relative velocity

volume flow rate

Weber number $W e=\varrho v^{2} R / \gamma$

variable/exponent

\section{Greek letters} attraction $F_{\gamma}=A_{h} /\left(\gamma R^{2}\right)$

$\operatorname{Re} \quad$ Reynolds number $R e=(v d \rho) / \mu$

$R e_{\varphi} \quad$ Reynolds number for droplet swarm
$\left[\mathrm{m}^{3} / \mathrm{s}\right] \quad \tau_{\text {bridg }}$
[N] $\varphi$
bridge formation time
$[\mathrm{s}]$
$\varphi$
$\xi$
phase fraction
collision frequency
[-]
$\left[\mathrm{m}^{3} / \mathrm{s}\right]$
$\left[\mathrm{m} / \mathrm{s}^{2}\right]$
$\left[\mathrm{s}^{-1}\right]$
[m]
[m]
Acknowledgments: The financial support provided by the German Research Foundation (DFG) within the project KR $1639 / 19-2$ is gratefully acknowledged.

[m]

[m]

[-]

[-]

$[-]$

[-]

[-]

$[-]$

[m]

[m]

[m] [m]

[m]

[m]

[m]

[s]

[s]

[s]

[s]

$[\mathrm{m} / \mathrm{s}]$

$[\mathrm{m} / \mathrm{s}]$

$[\mathrm{m} / \mathrm{s}]$

$\left[\mathrm{m}^{3} / \mathrm{s}\right]$

$[-]$

[various]

\section{References}

Aarts DGAL, Lekkerkerker HNW. Droplet coalescence: drainage, film rupture and neck growth in ultralow interfacial tension systems. J Fluid Mech 2008; 606: 275-294.

Aarts DGAL, Schmidt M, Lekkerkerker HNW. Direct visual observation of thermal capillary waves. Science 2004; 304: 847-850.

Aarts DGAL, Lekkerkerker HNW, Guo H, Wegdam GH, Bonn D. Hydrodynamics of droplet coalescence. Phys Rev Lett 2005; 95 : 164503.

Abid S, Chesters AK. The drainage and rupture of partially-mobile films between colliding drops at constant approach velocity. Int J Multiph Flow 1994; 20: 613-629.

Acevedo-Malavé A. Hydrodynamics coalescence collision of three liquid drops in 3D with smoothed particle hydrodynamics. AIP Adv 2012; 2: 42106.

Acevedo-Malavé A, Garcia-Sucre M. Coalescence collision of liquid drops. I: off-center collisions of equal-size drops. AIP Adv 2011a; 1: 32117.

Acevedo-Malavé A, Garcia-Sucre M. Coalescence collision of liquid drops. II: off-center collisions of unequal-size drops. AIP Adv 2011b; 1: 32118.

Agarwal S, von Arnim V, Stegmaier T, Planck H, Agarwal A. Role of surface wettability and roughness in emulsion separation. Sep Purif Technol 2013; 107: 19-25.

Aida K, Na YH, Nagaya T, Orihara H. Droplet coalescence process under electric fields in an immiscible polymer blend. Phys Rev E Stat Nonlinear Soft Matter Phys 2010; 82: 031805/1-031805/7.

Alder BJ, Wainwright TE. Studies in molecular dynamics. I. General method. J Chem Phys 1959; 31: 459.

Allak AMA, Jeffreys GV. Studies of coalescence and phase separation in thick dispersion bands. AIChE J 1974; 20: 564-570.
$[-]$

$[\mathrm{N} / \mathrm{m}]$

shear rate

difference

energy dissipation rate

coalescence efficiency/probability

dynamic viscosity

continuous phase dynamic viscosity

disperse phase dynamic viscosity

mean dispersion dynamic viscosity $\mu_{\varphi}=f\left(\varphi, \mu_{c}, \mu_{d}\right)$

dynamic viscosity ratio $\mu^{*}=\mu_{d} / \mu_{c}$

disjoining pressure

density

continuous phase density

disperse phase density

time

median rest (or coalescence) time

$\left[\mathrm{m}^{2} / \mathrm{s}^{3}\right]$

[Pa s]

[Pa s]

[Pa s]

[Pa s]

$\left[\mathrm{N} / \mathrm{m}^{2}\right]$

$\left[\mathrm{kg} / \mathrm{m}^{3}\right]$

$\left[\mathrm{kg} / \mathrm{m}^{3}\right]$

$\left[\mathrm{kg} / \mathrm{m}^{3}\right]$
Allan RS, Mason SG. Particle motions in sheared suspensions. XIV. Coalescence of liquid drops in electric and shear fields. J Colloid Sci 1962; 17: 383-408. gas/liquid interface. J Colloid Sci 1961; 16: 150-165.

Alopaeus V, Koskinen J, Keskinen KI. Simulation of the population balances for liquid-liquid systems in a nonideal stirred tank. Part 1. Description and qualitative validation of the model. Chem Eng Sci 1999; 54: 5887-5899. population balances for liquid-liquid systems in a nonideal stirred tank. Part 2. Parameter fitting and the use of the 57: 1815-1825.

Amoanu D. Studies of liquid-liquid separation in fibre coalescer. Ph.D. thesis. Lappeenranta University of Technology, 2008.
Allan RS, Charles GE, Mason SG. The approach of gas bubbles to a

Alopaeus V, Koskinen J, Keskinen KI, Majander J. Simulation of the multiblock model for dense dispersions. Chem Eng Sci 2002; 
Anderson JD. Computational fluid dynamics: the basics with applications, 1st ed., Weinheim: McGraw-Hill Science, 1995.

Aryafar H, Kavehpour HP. Drop coalescence through planar surfaces. Phys Fluids 2006; 18: 72105.

Ashgriz N, Givi P. Binary collision dynamics of fuel droplets. Int J Heat Fluid Flow 1987; 8: 205-210.

Ashgriz N, Givi P. Coalescence efficiencies of fuel droplets in binary collisions. Int Commun Heat Mass Transf 1989; 16: 11-20.

Ashgriz N, Poo JY. Coalescence and separation in binary collisions of liquid drops. J Fluid Mech 1990; 221: 183-204.

Ata S, Pugh RJ, Jameson GJ. The influence of interfacial ageing and temperature on the coalescence of oil droplets in water. Colloids Surf A Physicochem Eng Asp 2011; 374: 96-101.

Attarakih M. Integral formulation of the population balance equation: application to particulate systems with particle growth. Comput Chem Eng 2013; 48: 1-13.

Attarakih MM, Bart H-J, Faqir NM. Numerical solution of the spatially distributed population balance equation describing the hydrodynamics of interacting liquid-liquid dispersions. Chem Eng Sci 2004; 59: 2567-2592.

Attarakih MM, Bart H-J, Lagar GL, Faqir NM. LLECMOD: a Windows-based program for hydrodynamics simulation of liquid-liquid extraction columns. Chem Eng Process Process Intensif 2006; 45: 113-123.

Attarakih MM, Bart H-J, Steinmetz T, Dietzen M, Faqir NM. LLECMOD: a bivariate population balance simulation tool for liquid-liquid extraction columns. Open Chem Eng J 2008; 2: 10-34.

Attarakih M, Al-Zyod S, Abu-Khader M, Bart HJ. PPBLAB: a new multivariate population balance environment for particulate system modelling and simulation. Procedia Eng 2012; 42: $1445-1462$.

Baldessari F, Leal LG. Effect of overall drop deformation on flow-induced coalescence at low capillary numbers. Phys Fluids 2006; 18: 13602.

Ballard DH, Brown CM. Computer vision. NJ: Prentice-Hall, 1982.

Ban T, Kawaizumi F, Nii S, Takahashi K. Study of drop coalescence behavior for liquid-liquid extraction operation. Chem Eng Sci 2000; 55: 5385-5391.

Bancroft WD. The theory of emulsification. V J Phys Chem 1913; 17 : 501-519.

Bansal S, von Arnim V, Stegmaier T, Planck H. Effect of fibrous filter properties on the oil-in-water-emulsion separation and filtration performance. J Hazard Mater 2011; 190: 45-50.

Barnea E. Flooding conditions in a deep-layer liquid/liquid settler. Trans Inst Chem Eng [Chem Eng Res Des] 1978; 56: 73-76.

Barnea E, Mizrahi J. A generalized approach to the fluid dynamics of particulate systems. 1. General correlation for fluidization and sedimentation in solid multiparticle systems. Chem Eng J 1973; 5: 171-189.

Barnea E, Mizrahi J. Generalized approach to fluid-dynamics of particulate systems. 2. Sedimentation and fluidization of clouds of spherical liquid drops. Can J Chem Eng 1975a; 53: 461-468.

Barnea E, Mizrahi J. Separation mechanism of liquid-liquid dispersions in a deep-layer gravity settler. 2 . Flow patterns of dispersed and continuous phases within dispersion band. Trans Inst Chem Eng [Chem Eng Res Des] 1975b; 53: 70-74.

Barnea E, Mizrahi J. Separation mechanism of liquid-liquid dispersions in a deep-layer gravity settler. 4. Continuous settler characteristics. Trans Inst Chem Eng [Chem Eng Res Des] 1975c; 53: 83-92.
Barnea E, Mizrahi J. Separation mechanism of liquid-liquid dispersions in a deep-layer gravity settler. 1 . Structure of dispersion band. Trans Inst Chem Eng [Chem Eng Res Des] 1975d; 53: 61-69.

Barnea E, Mizrahi J. Separation mechanism of liquid-liquid dispersions in a deep-layer gravity settler. 3. Hindered settling and drop-to-drop coalescence in dispersion band. Trans Inst Chem Eng [Chem Eng Res Des] 1975e; 53: 75-82.

Bart H-J, Garthe D, Grömping T, Pfennig A, Schmidt S, Stichlmair J. Vom Einzeltropfen zur Extraktionskolonne [in German]. Chem Ing Tech 2006; 78: 543-547.

Bartok W, Mason SG. Particle motions in sheared suspensions. J Colloid Sci 1959; 14: 13-26.

Basheva ES, Gurkov TD, Ivanov IB, Bantchev GB, Campbell B, Borwankar RP. Size dependence of the stability of emulsion drops pressed against a large interface. Langmuir 1999; 15 : 6764-6769.

Batchelor GK. Pressure fluctuations in isotropic turbulence. Math Proc Camb Philos Soc 1951; 47: 359.

Batchelor GK. Turbulent motion. Rep Prog Phys 1952; 15: 101-141.

Batchelor GK, Green JT. The hydrodynamic interaction of two small freely-moving spheres in a linear flow field. J Fluid Mech 1972; 56: 375.

Bayraktar E, Mierka O, Platte F, Kuzmin D, Turek S. Numerical aspects and implementation of population balance equations coupled with turbulent fluid dynamics. Comput Chem Eng 2011; 35: 2204-2217.

Bazhlekov IB, van de Vosse FN, Chesters AK. Drainage and rupture of a Newtonian film between two power-law liquid drops interacting under a constant force. J Nonnewton Fluid Mech 2000; 93: 181-201.

Bazhlekov IB, Anderson PD, Meijer HEH. Nonsingular boundary integral method for deformable drops in viscous flows. Phys Fluids 2004; 16: 1064.

Berger R. Koaleszenzprobleme in chemischen Prozessen [in German]. Chem Ing Tech 1986; 58: 449-456.

Berman Y, Tamir A. Kinetics of droplets' sedimentation in a continuous gravity settler. Chem Eng Sci 2003; 58: 2089-2102.

Bhardwaj A, Hartland S. Kinetics of coalescence of water droplets in water-in-crude oil emulsions. J Dispers Sci Technol 1994; 15: 133-146.

Binnig G, Quate CF, Gerber C. Atomic force microscope. Phys Rev Lett 1986; 56: 930-933.

Black W, de Jongh JGV, Overbeek JTG, Sparnaay MJ. Measurements of retarded van der Waals-forces. Trans Faraday Soc 1960; 56: 1597.

Blanchette F, Bigioni TP. Partial coalescence of drops at liquid interfaces. Nat Phys 2006; 2: 254-257.

Bommaganti PK, Kumar MV, Ghosh P. Effects of binding of counterions on adsorption and coalescence. Chem Eng Res Des 2009; 87: 728-738.

Borges B, Rondon M, Sereno O, Asuaje J. Breaking of water-incrude-oil emulsions. 3. Influence of salinity and water-oil ratio on demulsifier action. Energy Fuels 2009; 23: 1568-1574.

Borrell M, Leal LG. Viscous coalescence of expanding low-viscosity drops; the dueling drops experiment. J Colloid Interface Sci 2008; 319: 263-269.

Borrell M, Yoon Y, Leal LG. Experimental analysis of the coalescence process via head-on collisions in a time-dependent flow. Phys Fluids 2004; 16: 3945-3954. 
Bozzano G, Dente M. Modelling the drop coalescence at the interface of two liquids. Comput Chem Eng 2011; 35: 901-906.

Bozzano G, Dente M. Coalescence between adjacent drops relying on the interface of two liquids. In: Pierucci S, editor. AIDIC Conference Series, 2013: 71-80.

Bradley SG, Stow CD. Collisions between liquid drops. Philos Trans R Soc Lond Ser A Math Phys Sci 1978; 287: 635-675.

Bremond N, Thiam AR, Bibette J. Decompressing emulsion droplets favors coalescence. Phys Rev Lett 2008; 100: 24501.

Brenn G, Frohn A. Collision and merging of two equal droplets of propanol. Exp Fluids 1989; 7: 441-446.

Brenn G, Kolobaric V. Satellite droplet formation by unstable binary drop collisions. Phys Fluids 2006; 18: 87101.

Brenn G, Kalenderski S, Ivanov I. Investigation of the stochastic collisions of drops produced by Rayleigh breakup of two laminar liquid jets. Phys Fluids 1997; 9: 349.

Brenn G, Valkovska D, Danov KD. The formation of satellite droplets by unstable binary drop collisions. Phys Fluids 2001; 13: 2463.

De Bruyn P, Cardinaels R, Moldenaers P. The effect of geometrical confinement on coalescence efficiency of droplet pairs in shear flow. J Colloid Interface Sci 2013; 409: 183-192.

Buchbender F, Onink F, Meindersma W, de Haan A, Pfennig A. Simulation of aromatics extraction with an ionic liquid in a pilot-plant Kühni extractor based on single-drop experiments. Chem Eng Sci 2012; 82: 167-176.

Burrill KA, Woods DR. Change in interface and film shapes for a deformable drop at a deformable liquid-liquid interface. J Colloid Interface Sci 1969; 30: 511-524.

Burrill KA, Woods DR. Film shapes for deformable drops at liquid-liquid interfaces. II. The mechanisms of film drainage. J Colloid Interface Sci 1973a; 42: 15-34.

Burrill KA, Woods DR. Film shapes for deformable drops at liquid-liquid interfaces. III. Drop rest-times. J Colloid Interface Sci 1973b; 42: 35-51.

Butt H-J, Graf K, Kappl M. Physics and chemistry of interfaces (Physics Textbook), Weinheim: Wiley-VCH, 2003. doi: $10.1002 / 3527602313$

Butt H-J, Cappella B, Kappl M. Force measurements with the atomic force microscope: technique, interpretation and applications. Surf Sci Rep 2005; 59: 1-152.

Carneiro JNE, Kaufmann V, Polifke W. Implementation of a moments model in OpenFOAM for polydispersed multiphase flows. In: Proceedings of Open Source CFD International Conference, Berlin, Germany, 2008.

Carnie SL, Chan DYC, Lewis C, Manica R, Dagastine RR. Measurement of dynamical forces between deformable drops using the atomic force microscope. I. Theory. Langmuir 2005; 21: 2912-2922.

Carroll BJ. The stability of emulsions and mechanisms of emulsion breakdown. In: Matijevic E, editor. Surface and colloid science. New York: Wiley-Interscience, 1976: 1-68.

Case S. Coalescence of low-viscosity fluids in air. Phys Rev E Stat Nonlinear Soft Matter Phys 2009; 79: 026307.

Case S, Nagel S. Coalescence in low-viscosity liquids. Phys Rev Lett 2008; 100: 084503.

Chan DYC, Klaseboer E, Manica R. Film drainage and coalescence between deformable drops and bubbles. Soft Matter 2011; 7: 2235.

Chappelear DC. Models of a liquid drop approaching an interface. J Colloid Sci 1961; 16: 186-190.
Charles GE, Mason SG. The mechanism of partial coalescence of liquid drops at liquid/liquid interfaces. J Colloid Sci 1960a; 15: 105-122.

Charles GE, Mason SG. The coalescence of liquid drops with flat liquid/liquid interfaces. J Colloid Sci 1960b; 15: 236-267.

Cheesman DF, King A. The electrical double layer in relation to the stabilisation of emulsions with electrolytes. Trans Faraday Soc 1940; 35: 241-247.

Chen J-D. A model of coalescence between two equal-sized spherical drops or bubbles. J Colloid Interface Sci 1985; 107: 209-220.

Chen R-H, Chen C-T. Collision between immiscible drops with large surface tension difference: diesel oil and water. Exp Fluids 2006; 41: 453-461.

Chen S, Doolen GD. Lattice Boltzmann method for fluid flows. Annu Rev Fluid Mech 1998; 30: 329-364.

Chen D, Pu B. Studies on the binary coalescence model. II. Effects of drops size and interfacial tension on binary coalescence time. J Colloid Interface Sci 2001; 243: 433-443.

Chen Y, Wang C. Hydrodynamic interaction of two deformable drops in confined shear flow. Phys Rev E Stat Nonlinear Soft Matter Phys 2014; 90: 033010.

Chen C-T, Maa J-R, Yang Y-M, Chang C-H. Effects of electrolytes and polarity of organic liquids on the coalescence of droplets at aqueous-organic interfaces. Surf Sci 1998; 406: 167-177.

Chen N, Kuhl T, Tadmor R, Lin Q, Israelachvili J. Large deformations during the coalescence of fluid interfaces. Phys Rev Lett 2004; 92: 24501.

Chen X, Mandre S, Feng JJ. An experimental study of the coalescence between a drop and an interface in Newtonian and polymeric liquids. Phys Fluids 2006; 18: 92103.

Chen D, Cardinaels R, Moldenaers P. Effect of confinement on droplet coalescence in shear flow. Langmuir 2009; 25: 12885-12893.

Chesters AK. The modeling of coalescence processes in fluid-liquid dispersions: a review of current understanding. Trans Inst Chem Eng [Chem Eng Res Des] 1991; 69: 259-270.

Chesters AK, Hofman G. Bubble coalescence in pure liquids. Appl Sci Res 1982; 38: 353-361.

Chevaillier JP, Klaseboer E, Masbernat O, Gourdon C. Effect of mass transfer on the film drainage between colliding drops. J Colloid Interface Sci 2006; 299: 472-485.

Clift R, Grace JR, Weber ME. Bubbles, drops and particles. New York: Academic Press, 1978.

Clunie JS, Goodman JF, Ingram BT. Thin liquid films. Surf Colloid Sci 1971; 3: 167-239.

Cockbain EG, McRoberts TS. The stability of elementary emulsion drops and emulsions. J Colloid Sci 1953; 8: 440-451.

Coons JE, Halley PJ, McGlashan SA, Tran-Cong T. Scaling laws for the critical rupture thickness of common thin films. Colloids Surf A Physicochem Eng Asp 2005; 263: 258-266.

Coulaloglou CA, Tavlarides LL. Description of interaction processes in agitated liquid-liquid dispersions. Chem Eng Sci 1977; 32: 1289-1297.

Creux P, Lachaise J, Graciaa A, Beattie JK, Djerdjev AM. Strong specific hydroxide ion binding at the pristine oil/water and air/ water interfaces. J Phys Chem B 2009; 113: 14146-14150.

Dagastine RR. Dynamic forces between two deformable oil droplets in water. Science 2006; 313: 210-213.

Dagastine RR, Stevens GW, Chan DYC, Grieser F. Forces between two oil drops in aqueous solution measured by AFM. J Colloid Interface Sci 2004; 273: 339-342. 
Dagastine RR, Webber GB, Manica R, Stevens GW, Grieser F, Chan DYC. Viscosity effects on hydrodynamic drainage force measurements involving deformable bodies. Langmuir 2010; 26: 11921-11927.

Danner T, Schubert H. Coalescence processes in emulsions. In: Dickinson E, Miller R, editors. Food colloids: fundamentals of formulation. Royal Society of Chemistry, 2001: 116-124. doi: 10.1039/9781847550842-00116

Danov KD, Petsev DN, Denkov ND, Borwankar R. Pair interaction energy between deformable drops and bubbles. J Chem Phys 1993; 99: 7179.

Davies GA. Recent development in coalescers for separation of liquids. CHEMSA 1982; 8: 6-7.

Davies GA, Jeffreys GV. Coalescence of droplets in packings - factors affecting the separation of droplet dispersion. Filtr Sep 1969; 6: 349-354.

Davies GA, Jeffreys GV, Smith DV. Rate of coalescence of the dispersed phase in a laboratory mixer settler unit. II. Analysis of coalescence in a ontinuous mixer settler system by a differential model. AIChE J 1970; 16: 827-831.

Davis SS, Smith A. The stability of hydrocarbon oil droplets at the surfactant/oil interface. Colloid Polym Sci 1976; 254: 82-98.

Davis RH, Schonberg JA, Rallison JM. The lubrication force between two viscous drops. Phys Fluids A Fluid Dyn 1989; 1: 77-81.

de Vries AJ. Foam stability. Part IV. Kinetics and activation energy of film rupture. Recl Trav Chim Pays-Bas 1958a; 77: 383-399.

de Vries AJ. Foam stability. Part I. Structure and stability of foams. Recl Trav Chim Pays-Bas 1958b; 77: 81-91.

de Vries AJ. Foam stability. Part II. Gas diffusion in foams. Recl Trav Chim Pays-Bas 1958c; 77: 209-223.

de Vries AJ. Foam stability. Part III. Spontaneous foam destabilization resulting from gas diffusion. Recl Trav Chim Pays-Bas 1958d; 77: 283-296.

Decent SP, Sharpe G, Shaw AJ, Suckling PM. The formation of a liquid bridge during the coalescence of drops. Int J Multiph Flow 2006; 32: 717-738.

Delgado AV, González-Caballero F, Hunter RJ, Koopal LK. Measurement and interpretation of electrokinetic phenomena. J Colloid Interface Sci 2007; 309: 194-224.

Derjaguin BV, Abrikossova II. Direct measurements of molecular attraction of solids. J Phys Chem Solids 1958; 5: 1-10.

Derjaguin B, Kussakov M. Anomalous properties of thin polymolecular films. V. Acta Physicochim URSS 1939; 10: 25-44.

Derjaguin BV, Landau EM. Theory of the stability of strongly charged lyophobic sols and of the adhesion of strongly charged particles in solutions of electrolytes. Acta Physicochitnica URSS 1941; 14: 633-662. Reprint: Prog Surf Sci 1993: 30-59. doi: 10.1016/0079-6816(93)90013-L.

Derjaguin B, Prokhorov P. On the cause of the non-coalescence of liquid drops upon contact. C R Acad Sci URSS 1946; 54: 507.

Reprint: Prog Surf Sci 1993; 43: 273-276. doi: 10.1016/00796816(93)90036-u.

Derjaguin BV, Churaev NV, Muller VM. Surface forces. New York: Consultants Bureau, 1987.

Dickinson E, Murray BS, Stainsby G. Coalescence stability of emulsion-sized droplets at a planar oil-water interface and the relationship to protein film surface rheology. J Chem Soc Faraday Trans 1 Phys Chem Condens Phases 1988; 84: 871.

Diemer RB, Olson JH. A moment methodology for coagulation and breakage problems. Part 1. Analytical solution of the steady-state population balance. Chem Eng Sci 2002a; 57: 2193-2209.

Diemer RB, Olson JH. A moment methodology for coagulation and breakage problems. Part 2. Moment models and distribution reconstruction. Chem Eng Sci 2002b; 57: 2211-2228.

Doubliez L. The drainage and rupture of a non-foaming liquid film formed upon bubble impact with a free surface. Int J Multiph Flow 1991; 17: 783-803.

Drumm C. Coupling of computational fluid dynamics and population balance modelling for liquid-liquid extraction. Ph.D. thesis. Technische Universität Kaiserslautern, 2009.

Drumm C, Attarakih MM, Bart H-J. Coupling of CFD with DPBM for an RDC extractor. Chem Eng Sci 2009; 64: 721-732.

Drumm C, Attarakih M, Hlawitschka MW, Bart H-J. One-group reduced population balance model for CFD simulation of a pilot-plant extraction column. Ind Eng Chem Res 2010; 49: 3442-3451.

Duchemin L, Eggers J, Josserand C. Inviscid coalescence of drops. J Fluid Mech 2003; 487: 167-178.

Dupuy PM, Fernandino M, Jakobsen HA, Svendsen HF. Using Cahn-Hilliard mobility to simulate coalescence dynamics. Comput Math Appl 2010; 59: 246-2259.

Eckert NL, Gormely LS. Phase separation in an experimental mixer-settler. Trans Inst Chem Eng [Chem Eng Res Des] 1989; 67: 175-184.

Eckstein A, Vogelpohl A. Untersuchungen zur Tropfen-TropfenKoaleszenz [in German]. Chem Ing Tech 1999; 71: 480-483.

Eddi A, Winkels KG, Snoeijer JH. Influence of droplet geometry on the coalescence of low viscosity drops. Phys Rev Lett 2013; 111: 144502-1-144502-5.

Eggers J, Lister JR, Stone HA. Coalescence of liquid drops. J Fluid Mech 1999; 401: 293-310.

Eiswirth RT, Bart H-J. Experimental investigation of droplet-droplet coalescence in liquid-liquid systems. In: Proceedings of International Solvent Extraction Conference, Tucson, AZ, 2008.

Eiswirth RT, Bart H-J, Atmakidis T, Kenig EY. Experimental and numerical investigation of a free rising droplet. Chem Eng Process Process Intensif 2011; 50: 718-727.

Eiswirth RT, Bart H-J, Ganguli AA, Kenig EY. Experimental and numerical investigation of binary coalescence: liquid bridge building and internal flow fields. Phys Fluids 2012; 24: 62108.

Eow J. Electrostatic enhancement of coalescence of water droplets in oil: a review of the technology. Chem Eng J 2002; 85: 357-368.

Eow JS, Ghadiri M. Drop-drop coalescence in an electric field: the effects of applied electric field and electrode geometry. Colloids Surf A Physicochem Eng Asp 2003; 219: 253-279.

Eow JS, Ghadiri M, Sharif AO, Williams TJ. Electrostatic enhancement of coalescence of water droplets in oil: a review of the current understanding. Chem Eng J 2001; 84: 173-192.

Erk KA, Martin JD, Schwalbe JT, Phelan FR, Hudson SD. Shear and dilational interfacial rheology of surfactant-stabilized droplets. J Colloid Interface Sci 2012; 377: 442-449.

Estrade J-P, Carentz H, Lavergne G, Biscos Y. Experimental investigation of dynamic binary collision of ethanol droplets a model for droplet coalescence and bouncing. Int J Heat Fluid Flow 1999; 20: 486-491.

Fang CS, Chang BKL, Lai PMC, Klaila WJ. Microwave demulsification. Chem Eng Commun 1988; 73: 227-239.

Frankel SP, Mysels KJ. On the "dimpling" during the approach of two interfaces. J Phys Chem 1962; 66: 190-191. 
Franks GV, Djerdjev AM, Beattie JK. Absence of specific cation or anion effects at low salt concentrations on the charge at the oil/water interface. Langmuir 2005; 21: 8670-8674.

Frenklach M. Method of moments with interpolative closure. Chem Eng Sci 2002; 57: 2229-2239.

Frising T, Noik C, Dalmazzone C. The liquid-liquid sedimentation process: from droplet coalescence to technologically enhanced water-oil emulsion gravity separators: a review. J Dispers Sci Technol 2006; 27: 1035-1057.

Frising T, Noïk C, Dalmazzone C, Peysson Y, Palermo T. Contribution of the sedimentation and coalescence mechanisms to the separation of concentrated water-in-oil emulsions. J Dispers Sci Technol 2008; 29: 827-834.

Gäbler A. Experimentelle Untersuchungen, Modellierung und Simulation gerührter Flüssig/flüssig-Systeme mit veränderlichen Stoff- und Betriebsparametern [in German]. Ph.D. thesis. Technische Universität Berlin, Chair of Chemical and Process Engineering, 2007.

Gäbler A, Wegener M, Paschedag AR, Kraume M. The effect of pH on experimental and simulation results of transient drop size distributions in stirred liquid-liquid dispersions. Chem Eng Sci 2006; 61: 3018-3024.

Gac JM, Gradon L. A two-dimensional modeling of binary coalescence time using the two-color lattice-Boltzmann method. J Aerosol Sci 2011; 42: 355-363.

Gafonova OV, Yarranton HW. The stabilization of water-inhydrocarbon emulsions by asphaltenes and resins. J Colloid Interface Sci 2001; 241: 469-478.

Gaitzsch F. Koaleszenzphänomene in Wasser-in-Öl-in-WasserDoppelemulsionen [in German]. Ph.D. thesis. Technische Universität Berlin, Chair of Chemical and Process Engineering, 2014.

Gaitzsch F, Gäbler A, Kraume M. Analysis of droplet expulsion in stagnant single water-in-oil-in-water double emulsion globules. Chem Eng Sci 2011a; 66: 4663-4669.

Gaitzsch F, Kamp J, Kraume M, Gäbler A. Vergleich des Koaleszenzverhaltens ruhender und umströmter Wasser-in-Ölin-Wasser-Einzeltropfen. Chem Ing Tech 2011b; 83: 511-517.

Garti N. Double emulsions - scope, limitations and new achievements. Colloids Surf A Physicochem Eng Asp 1997; 123-124: 233-246.

Garti N. A new approach to improved stability and controlled release in double emulsions, by the use of graft-comb polymeric amphiphiles. Acta Polym 1998; 49: 606-616.

Ghosh P. A comparative study of the film-drainage models for coalescence of drops and bubbles at flat interface. Chem Eng Technol 2004; 27: 1200-1205.

Ghotli RA, Raman AAA, Ibrahim S, Baroutian S. Liquid-liquid mixing in stirred vessels: a review. Chem Eng Commun 2013; 200: 595-627.

Gilet T, Mulleners K, Lecomte JP, Vandewalle N, Dorbolo S. Critical parameters for the partial coalescence of a droplet. Phys Rev $E$ 2007; 75: 36303.

Gillespie T, Rideal EK. The coalescence of drops at an oil-water interface. Trans Faraday Soc 1956; 52: 173.

Giribabu K, Ghosh P. Adsorption of nonionic surfactants at fluid-fluid interfaces: importance in the coalescence of bubbles and drops. Chem Eng Sci 2007; 62: 3057-3067.

Golob J, Modic R. Coalescence of liquid/liquid dispersions in gravity settlers. Trans Inst Chem Eng [Chem Eng Res Des] 1977; 55: 207-211.
Gonzalez RC, Woods RE, Eddins SL. Digital image processing using MATLAB. India: Pearson Education, 2004.

Goodson M, Kraft M. Simulation of coalescence and breakage: an assessment of two stochastic methods suitable for simulating liquid-liquid extraction. Chem Eng Sci 2004; 59: 3865-3881.

Gorbatschew SW, Mustel ER. Über die untere Stabilitätsgrenze von Tropfen bei ihrem Zusammenprall [in German]. Kolloid Z 1935; 73: $20-24$.

Gorbatschew SW, Nikiforowa WM. Über die obere Stabilitätsgrenze von Tropfen bei ihrem Zusammenprall [in German]. Kolloid Z 1935; 73: 14-20.

Gourdon C, Casamatta G. Influence of mass-transfer direction on the operation of a pulsed sieve-plate pilot column. Chem Eng Sci 1991; 46: 2799-2808.

Greenspan D, Heath LF. Supercomputer simulation of the modes of colliding microdrops of water. J Phys D Appl Phys 1991; 24: 2121-2123.

Griffin WC. Classification of surface-active agents by "HLB." J Cosmet Sci 1949; 1: 311-326.

Grimes BA. Population balance model for batch gravity separation of crude oil and water emulsions. Part I. Model formulation. J Dispers Sci Technol 2012; 33: 578-590.

Groothuis H, Zuiderweg FJ. Influence of mass transfer on coalescence of drops. Chem Eng Sci 1960; 12: 288-289.

Guido S, Simeone M. Binary collision of drops in simple shear flow. J Fluid Mech 1998; 357: 1-20.

Ha JW, Yoon Y, Leal LG. The effect of compatibilizer on the coalescence of two drops in flow. Phys Fluids 2003; 15: 849.

Hagesaether L. Coalescence and break-up of drops and bubbles. Ph.D. thesis. NTNU Trondheim, 2002.

Hagesaether L, Jakobsen HA, Svendsen HF. Theoretical analysis of fluid particle collisions in turbulent flow. Chem Eng Sci 1999; 54: 4749-4755.

Hagesaether L, Jakobsen HA, Hjarbo K, Svendsen HF. A coalescence and breakup module for implementation in CFD-codes. In: Sauro Pierucci, editor. Computer aided chemical engineering. Elsevier. Vol. 8, 2000: 367-372. ISSN 1570-7946.

Hamaker HC. The London-van der Waals attraction between spherical particles. Phys IV 1937; 10: 1058-1072.

Hardt S. An extended volume-of-fluid method for micro flows with short-range interactions between fluid interfaces. Phys Fluids 2005; 17: 100601

Hardy W. Chemistry at interfaces. J Chem Soc Trans 1925; 127: 1207.

Hartland S. The coalescence of a liquid drop at a liquid-liquid interface. Part I. Drop shape. Trans Inst Chem Eng [Chem Eng Res Des] 1967a; 45: T97-101.

Hartland S. The coalescence of a liquid drop at a liquid-liquid interface. Part II. Film thickness. Trans Inst Chem Eng [Chem Eng Res Des] 1967b; 45: T102-108.

Hartland S. The coalescence of a liquid drop at a liquid-liquid interface. Part III. Film rupture. Trans Inst Chem Eng [Chem Eng Res Des] 1967c; 45: T109-114.

Hartland S. The shape of a fluid drop approaching an interface. Can J Chem Eng 1969; 47: 221-225.

Hartland S, Jeelani SAK. Choice of model for predicting the dispersion height in liquid/liquid gravity settlers from batch settling data. Chem Eng Sci 1987; 42: 1927-1938.

Hartland S, Robinson JD. Shape of liquid drops approaching a deformable liquid-liquid interface in three-phase systems with unequal densities. Kolloid Z Z Polym 1971; 245: 420-426. 
Hartland S, Robinson JD. Uniform film model for the gravitational approach of fluid drops to plane and deformable interfaces. Can J Chem Eng 1973; 51: 647-654.

Hartland S, Yang B, Jeelani SAK. Dimple formation in the thin film beneath a drop or bubble approaching a plane surface. Chem Eng Sci 1994; 49: 1313-1322.

Henschke M. Dimensionierung liegender Flüssig-Flüssig-Abscheider anhand diskontinuierlicher Absetzversuche, FortschrittBerichte VDI, Reihe 3 (Verfahrenstechnik), Nr. 379 [in German]. Ph.D. thesis. Düsseldorf: VDI Verlag, 1995.

Henschke M, Schlieper LH, Pfennig A. Determination of a coalescence parameter from batch-settling experiments. Chem Eng J 2002; 85: 369-378.

Hernández-Sánchez JF, Lubbers LA, Eddi A, Snoeijer JH. Symmetric and asymmetric coalescence of drops on a substrate. Phys Rev Lett 2012; 109: 184502-1-184502-5.

Hinze JO. Fundamentals of the hydrodynamic mechanism of splitting in dispersion processes. AIChE J 1955; 1: 289-295.

Hirt CW, Nichols BD. Volume of fluid (VOF) method for the dynamics of free boundaries. J Comput Phys 1981; 39: 201-225.

Hlawitschka MW. Computational fluid dynamics aided design of stirred liquid-liquid extraction columns. Ph.D. thesis. Technischen Universität Kaiserslautern, Chair of Separation Science and Technology, 2013.

Hodgson TD, Lee JC. The effect of surfactants on the coalescence of a drop at an interface. I. J Colloid Interface Sci 1969; 30: 94-108.

Hodgson TD, Woods DR. The effect of surfactants on the coalescence of a drop at an interface. II. J Colloid Interface Sci 1969; 30: 429-446.

Hool KO, Saunders RC, Ploehn HJ. Measurement of thin liquid film drainage using a novel high-speed impedance analyzer. Rev Sci Instrum 1998; 69: 3232-3239.

Hoover WG. Molecular dynamics. Lect. Notes Phys. Springer-Verlag, 1986. doi: 10.1007/bfb0020009

Hounslow MJ, Ryall RL, Marshall VR. A discretized population balance for nucleation, growth, and aggregation. AIChE J 1988; 34: 1821-1832.

Howarth WJ. Coalescence of drops in a turbulent flow field. Chem Eng Sci 1964; 19: 33-38.

Hsia MA, Tavlarides LL. A simulation model for homogeneous dispersions in stirred tanks. Chem Eng J 1980; 20: 225-236.

Hsu AS, Roy A, Leal LG. Drop-size effects on coalescence of two equal-sized drops in a head-on collision. J Rheol 2008; 52: 1291.

Hu YT, Pine DJ, Leal LG. Drop deformation, breakup, and coalescence with compatibilizer. Phys Fluids 2000; 12: 484.

Hulburt HM, Katz S. Some problems in particle technology. Chem Eng Sci 1964; 19: 555-574.

Inamuro T, Ogata T, Tajima S, Konishi N. A lattice Boltzmann method for incompressible two-phase flows with large density differences. J Comput Phys 2004; 198: 628-644.

Israelachvili J. Direct measurements of forces between surfaces in liquids at the molecular level. Proc Natl Acad Sci USA 1987; 84: 4722-4724.

Israelachvili JN. Intermolecular and surface forces, 2nd ed., London: Academic Press, 1991.

Israelachvili JN, Adams GE. Direct measurement of long range forces between two mica surfaces in aqueous $\mathrm{KNO}_{3}$ solutions. Nature 1976; 262: 774-776.

Israelachvili JN, McGuiggan PM. Adhesion and short-range forces between surfaces. Part I. New apparatus for surface force measurements. J Mater Res 1990; 5: 2223-2231.
Israelachvili JN, Tabor D. The measurement of van der Waals dispersion forces in the range 1.5 to $130 \mathrm{~nm}$. Proc R Soc A Math Phys Eng Sci 1972; 331: 19-38.

Israelachvili J, Min Y, Akbulut M, Alig A, Carver G, Greene W, Kristiansen K, Meyer E, Pesika N, Rosenberg K, Zeng H. Recent advances in the surface forces apparatus (SFA) technique. Rep Prog Phys 2010; 73: 36601.

Ivanov IB. Effect of surface mobility on the dynamic behavior of thin liquid films. Pure Appl Chem 1980; 52: 1241-1262.

Ivanov IB, Kralchevsky PA. Stability of emulsions under equilibrium and dynamic conditions. Colloids Surf A Physicochem Eng Asp 1997; 128: 155-175.

Ivanov IB, Danov KD, Kralchevsky PA. Flocculation and coalescence of micron-size emulsion droplets. Colloids Surf A Physicochem Eng Asp 1999; 152: 161-182.

Janssen PJA, Anderson PD. Modeling film drainage and coalescence of drops in a viscous fluid. Macromol Mater Eng 2011; 296: 238-248.

Jeelani SAK, Hartland S. Hydrodynamics of asymmetrical film thinning. Can J Chem Eng 1984; 62: 13-25.

Jeelani SAK, Hartland S. Prediction of steady state dispersion height from batch settling data. AIChE J 1985; 31: 711-720.

Jeelani SAK, Hartland S. Effect of interfacial mobility on thin film drainage. J Colloid Interface Sci 1994; 164: 296-308.

Jeelani SAK, Hartland S. Effect of surface mobility on collision of spherical drops. J Colloid Interface Sci 1998; 206: 83-93.

Jeelani SAK, Windhab EJ. Drop coalescence in planar extensional flow and gravity. Chem Eng Sci 2009; 64: 2718-2722.

Jeelani SAK, Benoist G, Joshi KS, Gunde R, Kellenberger D, Windhab EJ. Creaming and aggregation of particles in suspensions. Colloids Surf A Physicochem Eng Asp 2005a; 263: 379-389.

Jeelani SAK, Hosig R, Windhab EJ. Kinetics of low Reynolds number creaming and coalescence in droplet dispersions. AIChE J 2005b; 51: 149-161.

Jeffreys GV, Davies GA. Coalescence of liquid droplets and liquid dispersions. In: Hanson C, editor. Recent advances in liquid-liquid extraction. Oxford: Pergamon Press, 1971: 495-584. doi: 10.1016/B978-0-08-015682-8.50018-3

Jeffreys GV, Hawksley JL. Stepwise coalescence of a single droplet at an oil-water interface. J Appl Chem 1962; 12: 329-336.

Jeffreys GV, Hawksley JL. Coalescence of liquid droplets in two-component-two-phase systems. Part I. Effect of physical properties on the rate of coalescence. AIChE J 1965a; 11: 413-417.

Jeffreys GV, Hawksley JL. Coalescence of liquid droplets in two-component-two-phase systems. Part II. Theoretical analysis of coalescence rate. AIChE J 1965b; 11: 418-424.

Jeffreys GV, Lawson GB. Effect of mass transfer on the rate of coalescence of single drops at a plane interface. Trans Inst Chem Eng [Chem Eng Res Des] 1965; 43: T294-T298.

Jeffreys GV, Davies GA, Pitt K. Rate of coalescence of the dispersed phase in a laboratory mixer settler unit. I. AIChE J 1970; 16: 823-827.

Jia X, McLaughlin JB, Kontomaris K. Lattice Boltzmann simulations of drop coalescence and chemical mixing. Phys A Stat Mech Appl 2006; 362: 62-67.

Jiang YJ, Umemura A, Law CK. An experimental investigation on the collision behaviour of hydrocarbon droplets. J Fluid Mech 1992; 234: 171.

Jones JE. On the determination of molecular fields. II. From the equation of state of a gas. Proc R Soc A Math Phys Eng Sci 1924; 106: 463-477. 
Jones AF, Wilson SDR. The film drainage problem in droplet coalescence. J Fluid Mech 1978; 87: 263-288.

Kabalnov AS. Coalescence in emulsions. In: Binks BP, editor. Modern aspects of emulsion science. Cambridge: Royal Society of Chemistry, 1998: 205-260. doi: 10.1039/9781847551474-00205

Kalem M, Buchbender F, Pfennig A. Simulation of hydrodynamics in RDC extraction columns using the simulation tool "ReDrop." Chem Eng Res Des 2011; 89: 1-9.

Kalweit M, Drikakis D. Collision dynamics of nanoscale Lennard-Jones clusters. Phys Rev B Condens Matter Mater Phys 2006; 74: 235415/1-235415/16.

Kamp J, Kraume M. Influence of drop size and superimposed mass transfer on coalescence in liquid/liquid dispersions - test cell design for single drop investigations. Chem Eng Res Des 2014; 92: 635-643.

Kamp J, Kraume M. Coalescence efficiency model including electrostatic interactions in liquid/liquid dispersions. Chem Eng Sci 2015; 126: 132-142.

Kamp AM, Chesters AK, Colin C, Fabre J. Bubble coalescence in turbulent flows: a mechanistic model for turbulence-induced coalescence applied to microgravity bubbly pipe flow. Int J Multiph Flow 2001; 27: 1363-1396.

Karbaschi M, Taeibi Rahni M, Javadi A, Cronan CL, Schano KH, Faraji S, Won JY, Ferri JK, Krägel J, Miller R. Dynamics of drops formation, growth, oscillation, detachment, and coalescence. Adv Colloid Interface Sci 2014; 222: 413-424.

Karpitschka S, Riegler H. Sharp transition between coalescence and non-coalescence of sessile drops. J Fluid Mech 2014; 743: R1-1-R1-11.

Karpitschka S, Hanske C, Fery A, Riegler H. Coalescence and noncoalescence of sessile drops: impact of surface forces. Langmuir 2014; 30: 6826-6830.

Katalinic M. Über die Flüsigkeitskügelchen, welche auf der Oberfläche derselben Flüssigkeit schwimmen [in German]. Z Phys 1926; 38: 511-512.

Kavehpour HP. Coalescence of drops. Annu Rev Fluid Mech 2015; 47: 245-268.

Khatri NL, Andrade J, Baydak EN, Yarranton HW. Emulsion layer growth in continuous oil-water separation. Colloids Surf A Physicochem Eng Asp 2011; 384: 630-642.

Kim J, Longmire EK. Investigation of binary drop rebound and coalescence in liquids using dual-field PIV technique. Exp Fluids 2009; 47: 263-278.

Klaseboer E, Chevaillier JP, Gourdon C, Masbernat O. Film drainage between colliding drops at constant approach velocity: experiments and modeling. J Colloid Interface Sci 2000; 229: 274-285.

Kokal SL. Crude oil emulsions: a state-of-the-art review. SPE Prod Facil 2005; 20: 5-13.

Kolmogorov AN. The local structure of turbulence in incompressible viscous fluid for very large Reynolds numbers [in Russian]. Dokl Akad Nauk SSSR 1941; 30: 299. Reprint and translation: Proc R Soc Lond A.

Komrakova AE, Eskin D, Derksen JJ. Numerical study of turbulent liquid-liquid dispersions. AIChE J 2015; 61: 2618-2633.

Koplik J, Pal S, Banavar JR. Dynamics of nanoscale droplets. Phys Rev E Stat Nonlin Soft Matter Phys 2002; 65: 21504.

Kopriwa N. Quantitative Beschreibung von Koaleszenzvorgängen in Extraktionskolonnen [in German]. Ph.D. thesis. RWTH Aachen, 2014.
Kopriwa N, Buchbender F, Ayesteran J, Kalem M, Pfennig A. A critical review of the application of drop-population balances for the design of solvent extraction columns. I. Concept of solving drop-population balances and modelling breakage and coalescence. Solvent Extr Ion Exch 2012; 30: 683-723.

Kourio MJ, Gourdon C, Casamatta G. Study of drop-interface coalescence: drainage time measurement. Chem Eng Technol 1994; 17: 249-254.

Kralchevsky PA, Danov KD, Denkov ND. Chemical physics of colloid systems and interfaces. In: Birdi KS, editor. Handbook of surface and colloid chemistry. 3rd ed. Boca Raton: CRC Press, 2008: 197-377.

Kuboi R, Komasawa I, Otake T. Behavior of dispersed particles in turbulent liquid flow. J Chem Eng Jpn 1972a; 5: 349-355.

Kuboi R, Komasawa I, Otake T. Collision and coalescence of dispersed drops in turbulent liquid flow. J Chem Eng Jpn 1972b; 5: 423-424.

Kulkarni PS, Patel SU, Chase GG. Layered hydrophilic/hydrophobic fiber media for water-in-oil coalescence. Sep Purif Technol 2012; 85: 157-164.

Kumar MK, Mitra T, Ghosh P. Adsorption of ionic surfactants at liquid-liquid interfaces in the presence of salt: application in binary coalescence of drops. Ind Eng Chem Res 2006; 45: 7135-7143.

Kumar J, Peglow M, Warnecke G, Heinrich S, Tsotsas E, Mörl L, Hounslow M, Reynolds G. Numerical methods on population balances. In: Tsotsas E, Mujumdar AS, editors. Modern drying technology. 1. Computational Tools at Different Scales. Weinheim: Wiley-VCH Verlag GmbH \& Co. KGaA, 2007: 209-260.

Kyuchoukov G, Kounev R. On the coalescence effects in a batch mixer-settler. Chem Eng J 1998; 69: 63-67.

Lang SB, Wilke CR. A Hydrodynamic mechanism for the coalescence of liquid drops. II. Experimental studies. Ind Eng Chem Fundam 1971; 10: 341-352.

Lasheras JC, Eastwood C, Martinez-Bazan C, Montanes JL. A review of statistical models for the break-up of an immiscible fluid immersed into a fully developed turbulent flow. Int J Multiph Flow 2002; 28: 247-278.

Lawrence ASC, Mills OS. Kinetics of the coagulation of emulsions. Discuss Faraday Soc 1954; 18: 98.

Leal LG. Flow induced coalescence of drops in a viscous fluid. Phys Fluids 2004; 16: 1833.

Leal LG. Advanced transport phenomena: fluid mechanics and convective transport processes, Cambridge: Cambridge University Press, 2007.

Lee JC, Hodgson TD. Film flow and coalescence. I. Basic relations, film shape, and criteria for interface mobility. Chem Eng Sci 1968; 23: 1375-1397.

Lee HY, Oh JK, Lee DH. Interpretation of continuous settling behavior from batch settling data in a Versatic acid 10-water system. Hydrometallurgy 1993; 32: 273-286.

Lehr F, Mewes D. A transport equation for the interfacial area density applied to bubble columns. Chem Eng Sci 2001; 56: 1159-1166.

Lehr F, Millies M, Mewes D. Bubble-Size distributions and flow fields in bubble columns. AIChE J 2002; 48: 2426-2443.

Li D. Coalescence between two small bubbles or drops. J Colloid Interface Sci 1994; 163: 108-119.

Li J, Gu Y. Coalescence of oil-in-water emulsions in fibrous and granular beds. Sep Purif Technol 2005; 42: 1-13. 
Liao Y, Lucas D. A literature review of theoretical models for drop and bubble breakup in turbulent dispersions. Chem Eng Sci 2009; 64: 3389-3406.

Liao Y, Lucas D. A literature review on mechanisms and models for the coalescence process of fluid particles. Chem Eng Sci 2010; 65: 2851-2864.

Liao M-L, Ju S-P, Yang S-H. Coalescence behavior of water nanoclusters: temperature and size effects. J Phys Chem C 2007; 111: 6927-6932.

Liu S, Li D. Drop coalescence in turbulent dispersions. Chem Eng Sci 1999; 54: 5667-5675.

Lobo L, Ivanov I, Wasan D. Dispersion coalescence: kinetic stability of creamed dispersions. AIChE J 1993; 39: 322-334.

Lockie HJ, Manica R, Stevens GW, Grieser F, Chan DYC, Dagastine RR. Precision AFM measurements of dynamic interactions between deformable drops in aqueous surfactant and surfactant-free solutions. Langmuir 2011; 27: 2676-2685.

Lockie H, Manica R, Tabor RF, Stevens GW, Grieser F, Chan DYC, Dagastine RR. Anomalous pull-off forces between surfactant-free emulsion drops in different aqueous electrolytes. Langmuir 2012; 28: 4259-4266.

Loewenberg M, Hinch EJ. Collision of two deformable drops in shear flow. J Fluid Mech 1997; 338: 299-315.

Loglio G, Pandolfini P, Ravera F, Pugh R, Makievski A V, Javadi A, Miller R. Experimental observation of drop-drop coalescence in liquid-liquid systems: instrument design and features. Prog Colloid Interface Sci (Bubble Drop Interfaces) 2011; 2: 385-400.

Luo H. Coalescence, breakup and liquid circulation in bubble column reactors. Ph.D. thesis. Trondheim: The Norwegian Institute of Technology, 1993.

Luo X, Jiang P, Fan L-S. High-pressure three-phase fluidization: hydrodynamics and heat transfer. AIChE J 1997; 43: 2432-2445.

Lyklema J. Fundamentals of interface and colloid science. Vol. III. Liquid-fluid interfaces. Fundamentals of interface and colloid science. London: Academic Press, 2000.

Maaß S. Experimental analysis, modeling and simulation of drop breakage in agitated turbulent liquid/liquid-dispersions. Ph.D. thesis. Technische Universität Berlin, Chair of Chemical and Process Engineering, 2011.

Maaß S, Kraume M. Determination of breakage rates using single drop experiments. Chem Eng Sci 2012; 70: 146-164.

MacKay GDM, Mason SG. Some effects of interfacial diffusion on the gravity coalescence of liquid drops. J Colloid Sci 1963a; 18: 674-683.

MacKay GDM, Mason SG. The gravity approach and coalescence of fluid drops at liquid interfaces. Can J Chem Eng 1963b; 41: 203-212.

MacKay GDM, Mason SG. Particle motions in sheared suspensions. Kolloid Z Z Polym 1964; 195: 138-148.

Mahajan LD. Eine Theorie der Erscheinung von flüssigen Tropfen auf der Oberfläche derselben Flüssigkeit [in German]. Kolloid Z 1933; 65: 20-23.

Mahajan LD. Über die Lebensdauer von flüssigen Tropfen auf der Oberfläche der gleichen Flüssigkeit [in German]. Kolloid Z 1934; 69: 16-21.

Majumder A, Kariwala V, Ansumali S, Rajendran A. Lattice Boltzmann method for population balance equations with simultaneous growth, nucleation, aggregation and breakage. Chem Eng Sci 2012; 69: 316-328.
Malmazet E de, Risso F, Masbernat O, Pauchard V. Coalescence of contaminated water drops at an oil/water interface: influence of micro-particles. Colloids Surf A Physicochem Eng Asp 2015; 482: 514-528.

Manev ED, Angarska JK. Critical thickness of thin liquid films: comparison of theory and experiment. Colloids Surf A Physicochem Eng Asp 2005; 263: 250-257.

Manev ED, Nguyen AV. Critical thickness of microscopic thin liquid films. Adv Colloid Interface Sci 2005; 114-115: 133-146.

Manev E, Tsekov R, Radoev B. Effect of thickness non-homogeneity on the kinetic behaviour of microscopic foam film. J Dispers Sci Technol 1997; 18: 769-788.

Manica R, Connor JN, Dagastine RR, Carnie SL, Horn RG, Chan DYC. Hydrodynamic forces involving deformable interfaces at nanometer separations. Phys Fluids 2008a; 20: 32101.

Manica R, Klaseboer E, Chan DYC. Dynamic interactions between drops - a critical assessment. Soft Matter 2008b; 4: 1613.

Mansouri A, Arabnejad H, Mohan RS. Numerical investigation of droplet-droplet coalescence and droplet-interface coalescence. In: ASME 2014 4th Joint US-European Fluids Engineering Division Summer Meeting. ASME International, 2014. doi: 10.1115/fedsm2014-21642

Marchisio DL, Vigil DR, Fox RO. Implementation of the quadrature method of moments in CFD codes for aggregation-breakage problems. Chem Eng Sci 2003a; 58: 3337-3351.

Marchisio DL, Vigil RD, Fox RO. Quadrature method of moments for aggregation-breakage processes. J Colloid Interface Sci 2003b; 258: 322-334.

Marinova KG, Alargova RG, Denkov ND, Velev OD, Petsev DN, Ivanov IB, Borwankar RP. Charging of oil-water interfaces due to spontaneous adsorption of hydroxyl ions. Langmuir 1996; 12: 2045-2051.

Mashayek F, Ashgriz N, Minkowycz WJ, Shotorban B. Coalescence collision of liquid drops. Int J Heat Mass Transf 2003; 46: 77-89.

Mason SL, May K, Hartland S. Drop size and concentration profile determination in petroleum emulsion separation. Colloids Surf A Physicochem Eng Asp 1995; 96: 85-92.

Mason LR, Stevens GW, Harvie DJE. Multi-scale volume of fluid modelling of droplet coalescence. In: Solnordal CB, Liovic P, Delaney GW, Witt PJ, editors. The 9th International Conference on CFD in the Minerals and Process Industries, 2012: 1-6.

Mason LR, Stevens GW, Harvie DJE. Subgrid CFD film-drainage modelling: application to buoyancy-driven droplet-wall collisions in emulsions. In: 19th Australasian Fluid Mechanics Conference, 2014: 1-5.

Meissner HP, Chertow B. Accelerated breaking of unstable emulsions. Ind Eng Chem 1946; 38: 856-859.

Meleán Y, Sigalotti LDG. Coalescence of colliding van der Waals liquid drops. Int J Heat Mass Transf 2005; 48: 4041-4061.

Menchaca-Rocha A, Martinez-Davalos A, Nunez R, Popinet S, Zaleski $S$. Coalescence of liquid drops by surface tension. Phys Rev $E$ Stat Nonlinear Soft Matter Phys 2001; 63: 046309-1-046309-5.

Mikula RJ, Munoz VA. Characterization of demulsifiers. In: Schramm LL, editor. Surfactants: fundamentals and applications in the petroleum industry. Cambridge: Cambridge University Press, 2000: 51-78. doi: 10.1017/cbo9780511524844

Mohamed-Kassim Z, Longmire EK. Drop coalescence through a liquid/liquid interface. Phys Fluids 2004; 16: 2170.

Mohammadi M, Shahhosseini S, Bayat M. Direct numerical simulation of water droplet coalescence in the oil. Int J Heat Fluid Flow 2012; 36: 58-71. 
Müller E, Berger R, Blass E, Sluyts D, Pfennig A. Liquid-liquid extraction. Ullmanns Encycl Ind Chem. Weinheim: Wiley-VCH 2008: 250-307. doi: 10.1002/14356007.b03_06.pub2.

Nachtigall S, Zedel D, Kraume M. Analysis of drop deformation dynamics in turbulent flow. Chin J Chem Eng 2015; 24: 264-277.

Nadiv C, Semiat R. Batch settling of liquid-liquid dispersion. Ind Eng Chem Res 1995; 34: 2427-2435.

Nobari MR, Jan Y-J, Tryggvason G. Head-on collision of drops - a numerical investigation. Phys Fluids 1996; 8: 29.

Noïk C, Chen J, Dalmazzone C. SPE-103808-PP electrostatic demulsification on crude oil: a state-of-the-art review. In: SPE International Oil \& Gas Conference and Exhibition, Beijing, China, 2006: 1-12.

Noïk C, Palermo T, Dalmazzone C. Modeling of liquid/liquid phase separation: application to petroleum emulsions. J Dispers Sci Technol 2013; 34: 1029-1042.

Orme M. Experiments on droplet collisions, bounce, coalescence and disruption. Prog Energy Combust Sci 1997; 23: 65-79.

Ortiz-Duenas C, Kim J, Longmire EK. Investigation of liquid-liquid drop coalescence using tomographic PIV. Exp Fluids 2010; 49: 111-129.

Osher S, Sethian JA. Fronts propagating with curvature-dependent speed: algorithms based on Hamilton-Jacobi formulations. J Comput Phys 1988; 79: 12-49.

Ostwald W. Beiträge zur Kenntnis der Emulsionen [in German]. Z Chem Ind Kolloide 1910; 6: 103-109.

Overbeek JTG, Sparnaay MJ. Experiments on long-range attractive forces between macroscopic objects. J Colloid Sci 1952; 7: 343-345.

Pan K-L, Chou P-C, Tseng Y-J. Binary droplet collision at high Weber number. Phys Rev E 2009; 80: 36301-36308.

Pangu GD, Feke DL. Acoustically aided separation of oil droplets from aqueous emulsions. Chem Eng Sci 2004; 59: 3183-3193.

Pawar AB, Caggioni M, Ergun R, Hartel RW, Spicer PT. Arrested coalescence in Pickering emulsions. Soft Matter 2011; 7: $7710-7716$.

Pawar AB, Caggioni M, Hartel RW, Spicer PT. Arrested coalescence of viscoelastic droplets with internal microstructure. Faraday Discuss 2012; 158: 341-350.

Pereira JC, Delgado-Linares J, Scorzza C, Rondon M, Rodriguez $\mathrm{S}$, Salager J-L. Breaking of water-in-crude oil emulsions. 4. Estimation of the demulsifier surfactant performance to destabilize the asphaltenes effect. Energy Fuels 2011; 25: 1045-1050.

Pfennig A, Schwerin A. Influence of electrolytes on liquid-liquid extraction. Ind Eng Chem Res 1998; 37: 3180-3188.

Pfennig A, Schwerin A, Gaube J. Consistent view of electrolytes in aqueous two-phase systems. J Chromatogr B Biomed Sci Appl 1998; 711: 45-52.

Pickering SU. CXCVI. Emulsions. J Chem Soc Trans 1907; 91: 2001.

Podgorska W. Scale-up effects in coalescing dispersions comparison of liquid-liquid systems differing in interface mobility. Chem Eng Sci 2005; 60: 2115-2125.

Podgórska W, Baldyga J. Scale-up effects on the drop size distribution of liquid-liquid dispersions in agitated vessels. Chem Eng Sci 2001; 56: 741-746.

Pozrikidis C. Boundary integral and singularity methods for linearized viscous flow, XIth ed., Cambridge: Cambridge University Press, 1992.
Premnath KN, Abraham J. Simulations of binary drop collisions with a multiple-relaxation-time lattice-Boltzmann model. Phys Fluids 2005; 17: 122105.

Prince MJ, Blanch HW. Bubble coalescence and break-up in air-sparged bubble columns. AIChE J 1990; 36: 1485-1499.

Princen HM. Shape of a fluid drop at a liquid-liquid interface. J Colloid Sci 1963; 18: 178-195.

Princen HM. The equilibrium shape of interfaces, drops and bubbles. Rigid and deformable particles and interfaces. In: Matijevic E, editor. Surface and colloid science. New York: Wiley-Interscience, 1969: 1-84.

Prokhorov PS. The effects of humidity deficit on coagulation processes and the coalescence of liquid drops. Discuss Faraday Soc 1954; 18: 41.

Pu B, Chen D. Studies on the binary coalescence model: I. Jumping coalescence phenomenon. J Colloid Interface Sci 2001; 235: 1-3.

Qian J, Law CK. Regimes of coalescence and separation in droplet collision. J Fluid Mech 1997; 331: 59-80.

Radoev B, Scheludko A, Manev E. Critical thickness of thin liquid films: theory and experiment. J Colloid Interface Sci 1983; 95: 254-265.

Rambhau D. Zeta potential: a force that monitors dispersion stability. Indian J Pharm Educ 1978; 12: 140-151.

Ramkrishna D. Status of population balances. Rev Chem Eng 1985; 3: 49-95.

Ramkrishna D. Population balances: theory and applications to particulate systems in engineering, San Diego: Academic Press, 2000.

Ramkrishna D, Singh MR. Population balance modeling: current status and future prospects. Annu Rev Chem Biomol Eng 2014; 5: 123-146.

Ramsden W. Separation of solids in the surface-layers of solutions and "suspensions" (observations on surface-membranes, bubbles, emulsions, and mechanical coagulation). Prelim Account Proc R Soc Lond 1903; 72: 156-164.

Randolph AD, Larson MA. Transient and steady state size distributions in continuous mixed suspension crystallizers. AIChE J 1962; 8: 639-645.

Rayleigh L. The influence of electricity on colliding water drops. Proc R Soc Lond 1878; 28: 405-409.

Rehbinder P, Wenström E. Stabilisierende Wirkung von Adsorptionsschichten grenzflächenaktiver Stoffe auf disperse Systeme. II. Stabilität von Blasen und Tropfen an Trennungsflächen [in German]. Kolloid Z 1930; 53: 145-158.

Rehbinder P, Lagutkina L, Wenström E. Stabilisierende Wirkung grenzflächenaktiver Stoffe auf Suspension hydrophober und hydrophiler Pulver in Wasser und nichtwässrigen Dispersionsmitteln. I [in German]. Z Phys Chem A 1930; 146: 63-78.

Reichert MD, Walker LM. Coalescence behavior of oil droplets coated in irreversibly-adsorbed surfactant layers. J Colloid Interface Sci 2015; 449: 480-487.

Rekvig L, Frenkel D. Molecular simulations of droplet coalescence in oil/water/surfactant systems. J Chem Phys 2007; 127: 134701.

Reynolds 0. On the action of rain to calm the sea. Proc Lit Philos Soc Manchester 1875; 14. Reprint: Reynolds O. Papers on Mechanical and Physical Subjects. Vol. 1. Cambridge, 1900: 86-88.

Reynolds 0. On the floating of drops on the surface of water depending only on the purity of the surface. Proc Manchester 
Lit Philos Soc. Papers on Mechanical and Physical Subjects 1881; 21. Reprint: Reynolds O. Papers on Mechanical and Physical Subjects. Vol. 1. Cambridge, 1900: 413-414.

Reynolds 0 . On the theory of lubrication and its application to Mr. Beauchamp Tower's experiments, including an experimental determination of the viscosity of olive oil. Philos Trans R Soc Lond 1886; 177: 157-234.

Ribeiro MM, Regueiras PF, Guimarães MML, Madureira CMN, Cruz-Pinto JJC. Optimization of breakage and coalescence model parameters in a steady-state batch agitated dispersion. Ind Eng Chem Res 2011; 50: 2182-2191.

Ristenpart WD, McCalla PM, Roy RV, Stone HA. Coalescence of spreading droplets on a wettable substrate. Phys Rev Lett 2006; 97: 064501.

Ritcey GM. Crud in solvent extraction processing - a review of causes and treatment. Hydrometallurgy 1980; 5: 97-107.

Rondon M, Bouriat P, Lachaise J, Salager J-L. Breaking of water-in-crude oil emulsions. 1. Physicochemical phenomenology of demulsifier action. Energy Fuels 2006; 20: 1600-1604.

Rother MA, Zinchenko AZ, Davis RH. Buoyancy-driven coalescence of slightly deformable drops. J Fluid Mech 1997; 346: 117-148.

Ruiz MC, Padilla R. Separation of liquid-liquid dispersions in a deep-layer gravity settler. Part II. Mathematical modeling of the settler. Hydrometallurgy 1996; 42: 281-291.

Ryon AD, Lowrie RS. Experimental basis for design of mixer-settlers for the AMEX solvent extraction process 1963. Technical Report: ORNL-3381, Oak Ridge National Lab., Tenn. doi: $10.2172 / 4714591$.

Ryon AD, Daley FL, Lowrie RS. Design and scaleup of mixer-settlers for the DAPEX solvent extraction process 1960. Technical Report: ORNL-2951, Oak Ridge National Lab., Tenn. doi: $10.2172 / 4143506$.

Saboni A, Gourdon C, Chesters AK. Drainage and rupture of partially mobile films during coalescence in liquid-liquid systems under a constant interaction force. J Colloid Interface Sci 1995; 175: 27-35.

Saboni A, Alexandrova S, Gourdon C, Chesters AK. Interdrop coalescence with mass transfer: comparison of the approximate drainage models with numerical results. Chem Eng J 2002; 88: 127-139.

Saffman PG, Turner JS. On the collision of drops in turbulent clouds. J Fluid Mech 1956; 1: 16.

Sagert NH, Quinn MJ. The coalescence of $\mathrm{H}_{2} \mathrm{~S}$ and $\mathrm{CO}_{2}$ bubbles in water. Can J Chem Eng 1976; 54: 392-398.

Sagert NH, Quinn MJ. The coalescence of $n$-hexane droplets in aqueous electrolyte solutions. Can J Chem Eng 1978; 56: 679-684.

Sajjadi B, Raman AAA, Shah RSSRE, Ibrahim S. Review on applicable breakup/coalescence models in turbulent liquid-liquid flows. Rev Chem Eng 2013; 29: 131-158.

Sanyal J, Marchisio DL, Fox RO, Dhanasekharan K. On the comparison between population balance models for CFD simulation of bubble columns. Ind Eng Chem Res 2005; 44: 5063-5072.

Sata N, Harisaki Y. Über Koaleszenzerscheinungen grober Tropfensysteme [in German]. Kolloid Z 1960; 171: 101-107.

Sata N, Harisaki Y, Sasaki H. Über Koaleszenzerscheinung grober Tropfensysteme (WO-Typ) [in German]. Kolloid Z Z Polym 1964; 196: 53-57.
Scheele GF, Leng DE. An experimental study of factors which promote coalescence of two colliding drops suspended in water. I. Chem Eng Sci 1971; 26: 1867-1879.

Scheludko A. Über das Ausfließen der Lösung aus Schaumfilmen [in German]. Kolloid Z 1957; 155: 39-44.

Scheludko A, Exerowa D. Über den elektrostatischen Druck in Schaumfilmen aus wässerigen Elektrolytlösungen [in German]. Kolloid Z 1959; 165: 148-151.

Scheludko A, Platikanov D, Manev E. Disjoining pressure in thin liquid films and the electro-magnetic retardation effect of the molecule dispersion interactions. Discuss Faraday Soc 1965; 40: 253-265.

Schlieper L, Chatterjee M, Henschke M, Pfennig A. Liquid-liquid phase separation in gravity settler with inclined plates. AIChE J 2004; 50: 802-811.

Schramm LL. Emulsions, foams, and suspensions, Weinheim: Wiley-VCH, 2005.

Schwalbe JT, Phelan Jr FR, Vlahovska PM, Hudson SD. Interfacial effects on droplet dynamics in Poiseuille flow. Soft Matter 2011; 7: 7797-7804.

Shalhoub NG. Coalescence of secondary dispersions in fibrous beds. Ph.D. thesis. Department of Chemical Engineering, University of Aston Birmingham, 1975.

Shardt O, Derksen JJ, Mitra SK. Simulations of droplet coalescence in simple shear flow. Langmuir 2013; 29: 6201-6212.

Shardt O, Derksen JJ, Mitra SK. Simulations of Janus droplets at equilibrium and in shear. Phys Fluids 2014a; 26: 12104.

Shardt O, Mitra SK, Derksen JJ. The critical conditions for coalescence in phase field simulations of colliding droplets in shear. Langmuir 2014b; 30: 14416-14426.

Sheludko A. Thin liquid films. Adv Colloid Interface Sci 1967; 1: 391-464.

Sherony DF, Kintner RC, Wasan DT. Coalescence of secondary emulsions in fibrous beds. In: Matijevic E, editor. Surface and colloid science. New York: Wiley-Interscience, 1978: 99-161.

Silva LFLR, Rodrigues RC, Mitre JF, Lage PLC. Comparison of the accuracy and performance of quadrature-based methods for population balance problems with simultaneous breakage and aggregation. Comput Chem Eng 2010; 34: 286-297.

Simon M. Koaleszenz von Tropfen und Tropfenschwärmen [in German]. Ph.D. thesis. Technische Universität Kaiserslautern, Chair of Separation Science and Technology, 2004.

Simon M, Bart H-J. Experimental studies of coalescence in liquid-liquid systems. Chem Eng Technol 2002; 25: 481-484.

Smoluchowski Mv. Drei Vorträge über Diffusion, Brownsche Molekularbewegung und Koagulation [in German]. Phys Z 1916; 17: 557-599.

Smoluchowski M. Versuch einer mathematischen Theorie der Koagulationskinetik kolloider Lösungen [in German]. Z Phys Chem 1918; 92: 129-168.

Soika M, Pfennig A. Extraktion - Eine Frage des Wassers? [in German]. Chem Ing Tech 2005; 77: 905-911.

Solsvik J, Jakobsen HA. Bubble coalescence modeling in the population balance framework. J Dispers Sci Technol 2014; 35: 1626-1642.

Solsvik J, Jakobsen HA. Single drop breakup experiments in stirred liquid-liquid tank. Chem Eng Sci 2015a; 131: 219-234.

Solsvik J, Jakobsen HA. The foundation of the population balance equation: a review. J Dispers Sci Technol 2015b; 36: 510-520.

Solsvik J, Tangen S, Jakobsen HA. On the constitutive equations for fluid particle breakage. Rev Chem Eng 2013; 29: 242-356. 
Sovova H. Breakage and coalescence of drops in a batch stirred vessel. Il. Comparison of model and experiments. Chem Eng Sci 1981; 36: 1567-1573.

Speth H, Pfennig A, Chatterjee M, Franken H. Coalescence of secondary dispersions in fiber beds. Sep Purif Technol 2002; 29: 113-119.

Sporleder F, Borka Z, Solsvik J, Jakobsen HA. On the population balance equation. Rev Chem Eng 2012; 28: 149-169.

Stamm AJ, Kraemer EO. A note on the mechanism of emulsification. J Phys Chem 1925; 30: 992-1000.

Stevens GW, Pratt HRC, Tai DR. Droplet coalescence in aqueous electrolyte solutions. J Colloid Interface Sci 1990; 136: 470-479.

Sullivan AP, Kilpatrick PK. The effects of inorganic solid particles on water and crude oil emulsion stability. Ind Eng Chem Res 2002; 41: 3389-3404.

Sun K, Jia M, Wang T. Numerical investigation of head-on droplet collision with lattice Boltzmann method. Int J Heat Mass Transf 2013; 58: 260-275.

Sun K, Jia M, Wang T. Numerical investigation on the head-on collision between unequal-sized droplets with multiplerelaxation-time lattice Boltzmann model. Int J Heat Mass Transf 2014; 70: 629-640.

Svanberg M, Ming L, Markovic N, Pettersson JBC. Collision dynamics of large water clusters. J Chem Phys 1998; 108: 5888-5897.

Svendsen HVF, Luo H. Modeling of approach processes for equal or unequal sized fluid particles. Can J Chem Eng 1996; 74 : 321-330.

Tabor D, Winterton RHS. The direct measurement of normal and retarded van der Waals forces. Proc R Soc A Math Phys Eng Sci 1969; 312: 435-450.

Tabor RF, Lockie H, Mair D, Manica R, Chan DYC, Grieser F, Dagastine RR. Combined AFM-confocal microscopy of oil droplets: absolute separations and forces in nanofilms. J Phys Chem Lett 2011; 2: 961-965.

Tabor RF, Grieser F, Dagastine RR, Chan DYC. Measurement and analysis of forces in bubble and droplet systems using AFM. J Colloid Interface Sci 2012; 371: 1-14.

Tabor RF, Grieser F, Dagastine RR, Chan DYC. The hydrophobic force: measurements and methods. Phys Chem Chem Phys 2014; 16 : 18065.

Thomson JJ, Newall HF. On the formation of vortex rings by drops falling into liquids, and some allied phenomena. Proc R Soc Lond 1885; 39: 417-436.

Thoroddsen ST. Fluid dynamics: droplet genealogy. Nat Phys 2006; 2: 223-224.

Thoroddsen ST, Takehara K. The coalescence cascade of a drop. Phys Fluids 2000; 12: 1265-1267.

Thoroddsen ST, Takehara K, Etoh TG. The coalescence speed of a pendent and a sessile drop. J Fluid Mech 2005; 527: 85-114.

Thoroddsen ST, Etoh TG, Takehara K. High-speed imaging of drops and bubbles. Annu Rev Fluid Mech 2008; 40: 257-285.

Tobin T, Ramkrishna D. Coalescence of charged droplets in agitated liquid-liquid dispersions. AIChE J 1992; 38: 1199-1205.

Toro-Mendoza J, Petsev DN. Brownian dynamics of emulsion film formation and droplet coalescence. Phys Rev E Stat Nonlinear Soft Matter Phys 2010; 81: 51404.

Traykov TT, Ivanov IB. Hydrodynamics of thin liquid films. Effect of surfactants on the velocity of thinning of emulsion films. Int J Multiph Flow 1977; 3: 471-483.
Tretheway DC, Muraoka M, Leal LG. Experimental trajectories of two drops in planar extensional flow. Phys Fluids 1999; 11: 971.

Tsouris C, Tavlarides LL. Mass-transfer effects on droplet phenomena and extraction column hydrodynamics revisited. Chem Eng Sci 1993; 48: 1503-1515.

Tsouris C, Tavlarides LL. Breakage and coalescence models for drops in turbulent dispersions. AIChE J 1994; 40: 395-406.

Valentas KJ, Amundson NR. Breakage and coalescence in dispersed phase systems. Ind Eng Chem Fundam 1966; 5: 533-542.

Velev OD, Constantinides GN, Avraam DG, Payatakes AC, Borwankar RP. Investigation of thin liquid films of small diameters and high capillary pressures by a miniaturized cell. J Colloid Interface Sci 1995; 175: 68-76.

Verdier C. Coalescence of polymer droplets: experiments on collision. C R Acad Sci Ser IV Phys 1999; 1: 119-126.

Verdier C. The influence of the viscosity ratio on polymer droplet collision in quiescent blends. Polymer 2001; 42: 6999-7007.

Verdier C, Brizard M. Understanding droplet coalescence and its use to estimate interfacial tension. Rheol Acta 2002; 41: 514-523.

Verwey EJW, Overbeek JTG. Theory of the stability of lyophobic colloids, New York: Elsevier, 1948.

Vijayan S, Ponter AB. Inter-droplet coalescence in liquid and gas media. Tenside Deterg 1974; 11: 241-248.

Vijayan S, Ponter AB. Drop/drop and drop/interface coalescence in primary liquid/liquid dispersion separators. Chem Ing Tech 1975; 47: 748-755.

Villwock J, Gebauer F, Kamp J, Bart H-J, Kraume M. Systematic analysis of single droplet coalescence. Chem Eng Technol 2014a; 37: 1103-1111.

Villwock J, Kamp J, Kraume M. Systematic analysis of coalescence in liquid/liquid dispersions. In: Kraume M, Wehinger G, editors. Conference Proceedings of the 20th International Conference of Process Engineering and Chemical Plant Design 2014b: 119-126.

Vohra DK, Hartland S. Effect of geometrical arrangement and interdrop forces on coalescence time. Can J Chem Eng 1981; 59: 438-449.

Vrij A. Possible mechanism for the spontaneous rupture of thin, free liquid films. Discuss Faraday Soc 1966; 42: 23-33.

Vrij A, Hesselink FT, Lucassen J, den Tempel M. Waves in thin liquid films. II. Symmetrical modes in very thin films and film rupture. Proc K Ned Akad Wet Ser B Phys Sci 1970; 73: 124-135.

Wang W, Gong J, Ngan KH, Angeli P. Effect of glycerol on the binary coalescence of water drops in stagnant oil phase. Chem Eng Res Des 2009; 87: 1640-1648.

Wang B-B, Wang X-D, Yan W-M, Wang T-H. Molecular dynamics simulations on coalescence and non-coalescence of conducting droplets. Langmuir 2015; 31: 7457-7462.

Ward JD, Yu C-C. Population balance modeling in Simulink: PCSS. Comput Chem Eng 2008; 32: 2233-2242.

Wark IW, Cox AB. Coalescence in stages between two drops of a liquid. Nature 1935; 136: 182.

Watanabe A. Electrochemistry of oil-water interfaces. In: Matijevic E, editor. Surface and colloid science. New York: Wiley-Interscience, 1984: 1-70.

Watanabe T, Kusui M. On the mechanism of the coalescence of drops at an oil-water interface. Bull Chem Soc Jpn 1958; 31 236-243.

Webber GB, Edwards SA, Stevens GW, Grieser F, Dagastine RR, Chan DYC. Measurements of dynamic forces between drops with the AFM: novel considerations in comparisons between experiment and theory. Soft Matter 2008; 4: 1270-1278. 
Wegener M, Paul N, Kraume M. Fluid dynamics and mass transfer at single droplets in liquid/liquid systems. Int J Heat Mass Transf 2014; 71: 475-495.

Wesseling P. Principles of computational fluid dynamics. Springer Ser Comput Math. Vol. 29, Berlin: Springer, 2001: 644.

Willis KD, Orme ME. Experiments on the dynamics of droplet collisions in a vacuum. Exp Fluids 2000; 29: 347-358.

Willis $\mathrm{K}$, Orme $\mathrm{M}$. Binary droplet collisions in a vacuum environment: an experimental investigation of the role of viscosity. Exp Fluids 2003; 34: 28-41.

Wines TH, Brown RL. Difficult liquid-liquid separations. Chem Eng Mag 1997; 104: 104-109.

Wu M, Cubaud T, Ho C-M. Scaling law in liquid drop coalescence driven by surface tension. Phys Fluids 2004; 16: L51-L54.

Wulkow M, Gerstlauer A, Nieken U. Modeling and simulation of crystallization processes using parsival. Chem Eng Sci 2001; 56: 2575-2588.

Yang H, Park CC, Hu YT, Leal LG. The coalescence of two equal-sized drops in a two-dimensional linear flow. Phys Fluids 2001; 13: 1087-1106.

Yeo LY, Matar OK, de Ortiz ES, Hewitt GF. Film drainage between two surfactant-coated drops colliding at constant approach velocity. J Colloid Interface Sci 2003; 257: 93-107.

Yiantsios SG, Davis RH. Close approach and deformation of two viscous drops due to gravity and van der Waals forces. J Colloid Interface Sci 1991; 144: 412-433.

Yoon R-H, Aksoy BS. Hydrophobic forces in thin water films stabilized by dodecylammonium chloride. J Colloid Interface Sci 1999; 211: 1-10.

Yoon Y, Borrell M, Park CC, Leal LG. Viscosity ratio effects on the coalescence of two equal-sized drops in a two-dimensional linear flow. J Fluid Mech 2005; 525: 355-379.

Yoon Y, Baldessari F, Ceniceros HD, Leal LG. Coalescence of two equal-sized deformable drops in an axisymmetric flow. Phys Fluids 2007; 19: 102102.

Yu G-Z, Mao Z-S. Sedimentation and coalescence profiles in liquid-liquid batch settling experiments. Chem Eng Technol 2004; 27: 407-413.

Zdravkov AN, Peters GWM, Meijer HEH. Film drainage between two captive drops: PEO-water in silicon oil. J Colloid Interface Sci 2003; 266: 195-201.

Zdravkov AN, Peters GWM, Meijer HEH. Film drainage and interfacial instabilities in polymeric systems with diffuse interfaces. J Colloid Interface Sci 2006; 296: 86-94.

Zhang L, Xu B, Jiang B, Liu Y. Effect of electric double layer repulsion on oil droplet coalescence process. Chem Eng Technol 2010; 33: 878-884.

Zhao L, Choi P. Molecular dynamics simulation of the coalescence of nanometer-sized water droplets in $\mathrm{n}$-heptane. J Chem Phys 2004; 120: 1935-1942.

Zinchenko AZ, Rother MA, Davis RH. A novel boundary-integral algorithm for viscous interaction of deformable drops. Phys Fluids 1997; 9: 1493.

\section{Bionotes}

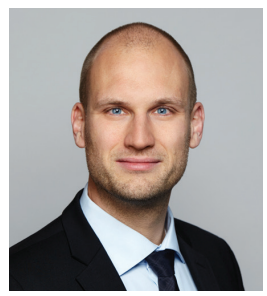

\section{Johannes Kamp}

Chair of Chemical and Process Engineering, Technische Universität Berlin, Straße des 17. Juni 135, FH 6-1, 10623 Berlin, Germany, Johannes.Kamp@tu-berlin.de

Johannes Kamp studied mechanical engineering at Ruhr-Universität Bochum (Germany) and graduated with a degree in biochemical engineering at RWTH Aachen (Germany). In 2009, he began his doctorate at the Chair of Chemical and Process Engineering at Technische Universität Berlin (Germany). His research focuses on the fundamentals of droplet coalescence in liquid/liquid dispersions and liquid/liquid separation using phase inversion.

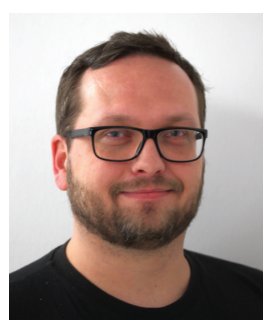

\section{Jörn Villwock}

Chair of Chemical and Process Engineering, Technische Universität Berlin, Straße des 17. Juni 135, FH 6-1, 10623 Berlin, Germany

Jörn Villwock studied and graduated with a degree in environmental engineering at HTW Berlin (Germany) and chemical engineering at Technische Universität Berlin (Germany). Since 2012, he has been a PhD student at the Chair of Chemical and Process Engineering at Technische Universität Berlin (Germany). His research focuses on coalescence in liquid/liquid systems under the influence of electrostatic forces.

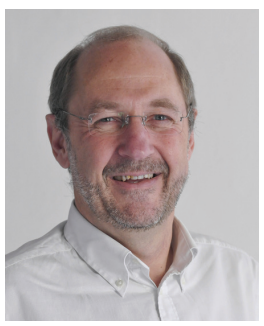

\section{Matthias Kraume}

Chair of Chemical and Process Engineering, Technische Universität Berlin, Straße des 17. Juni 135, FH 6-1, 10623 Berlin, Germany

Matthias Kraume studied chemical Engineering at universität Dortmund (Germany). In 1985, he received his PhD for his work on direct contact heat transfer. After finishing his PhD, he worked at BASF, Ludwigshafen, in the research and engineering departments. Since 1994, he has been a full professor at Technische Universität Berlin (Germany) and head of Chair of Chemical Engineering. His research fields include transport phenomena in multiphase systems, membrane processes, and reactor design. 


\section{Graphical abstract}

Johannes Kamp, Jörn Villwock and

Matthias Kraume

Drop coalescence in technical

liquid/liquid applications:

experimental techniques and

modeling approaches

DOI 10.1515/revce-2015-0071

Rev Chem Eng 2016; $x(x): x x x-x x x$
Review: The coalescence of drops in liquid/liquid systems is reviewed with a focus on its technical relevance, available experimental techniques, and modeling approaches.

Keywords: coalescence; coalescence model; emulsion; liquid/liquid dispersion; population balance equation.

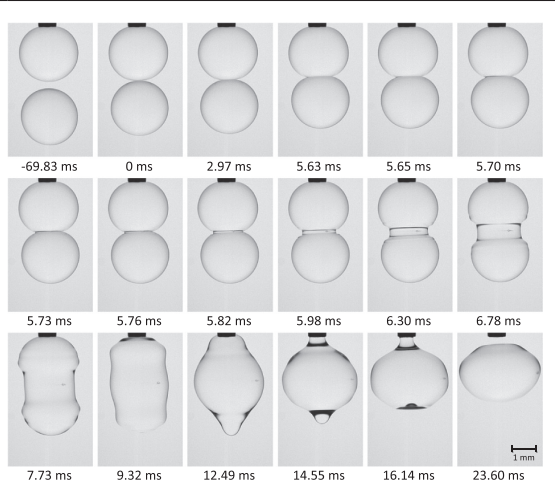

